\title{
ASYMPTOTIC EXPANSION OF EIGENELEMENTS OF THE LAPLACE OPERATOR IN A DOMAIN WITH A LARGE NUMBER OF 'LIGHT' CONCENTRATED MASSES SPARSELY SITUATED ON THE BOUNDARY. TWO-DIMENSIONAL CASE
}

\author{
G. A. CHECHKIN
}

\begin{abstract}
This paper looks at eigenoscillations of a membrane containing a large number of concentrated masses on the boundary. The asymptotic behaviour of the frequencies of eigenoscillations is studied when a small parameter characterizing the diameter and density of the concentrated masses tends to zero. Asymptotic expansions of eigenelements of the corresponding problems are constructed and the expansions are accurately substantiated. The case where the diameter of the masses is much smaller than the distance between them is investigated under the assumption that the limit boundary condition is still a Dirichlet condition.
\end{abstract}

\section{INTRODUCTION}

The behaviour of bodies with singular density has attracted the attention of scientists for many years. They have studied the influence of concentrated masses (singular lumps) on the variation of the frequencies of eigenoscillations of such bodies. This question proved to be rather too difficult for the mathematics available at the end of the 19th century. We must give due credit to a pioneering paper from the beginning of the 20th century [1; its author investigated the problem of the oscillation of a string loaded with concentrated masses. This paper was far ahead of its time and was forgotten for some years. It was only after asymptotic methods appeared that the interest of researchers returned to problems with concentrated masses and it became possible to investigate problems with singular density adequately.

The author of [2] considered the problem for the Laplace operator with Dirichlet boundary conditions in the three-dimensional case where the mass attached to the system is concentrated in an $\varepsilon$-neighbourhood of an interior point, $\varepsilon$ being a small parameter describing the concentration and size of the mass. In that paper the methods of spectral perturbation theory were used.

Another approach was proposed in [3, 4, 5, 6, 7]. As has already frequently been pointed out, a new basic parameter for oscillatory systems with locally attached masses was introduced in these papers, namely, the ratio of the attached mass to the mass of the whole system. This approach has long been firmly established in research papers. It turns out that the introduction of this parameter made it possible not only to analyse the

2000 Mathematics Subject Classification. Primary 35J25; Secondary 35B25, 35B27, 35B40.

Key words and phrases. Laplace operator, eigenoscillations, asymptotic expansions, singular perturbations.

This research was partially supported by the Russian Foundation for Basic Research (grant \# 09-0100530a) and by the Programme for Support of Leading Scientific Schools (grant \# NSh-1698.2008.1). 
limit (effective) behaviour of such problems, but also to construct complete asymptotic series for eigenelements, accurately substantiating them.

Dimensional analysis in the problem of the spectral properties of oscillatory systems with attached masses was first carried out in [8]. The case of a single concentrated mass for a one-dimensional operator with a Dirichlet boundary condition was studied in 9]. In the case of finitely many concentrated masses, this work was done in [10].

Later, various problems were considered for arbitrary domains, for thin plates and rods, for a variety differential operators and boundary conditions, for 'light', 'heavy' and 'critical density' masses. The problem of a rod and of a plate with concentrated masses were studied in [11, 12] (see also [13, 14, 15]). The case of a free boundary (Neumann conditions) was analysed in [16] and [17. In [18, the behaviour of eigenelements of the Laplace operator with a Dirichlet condition and a Fourier condition (a condition of the third kind) on the boundary was studied in the case where the density is perturbed by finitely many concentrated masses; see also [19. The question of constructing asymptotic expansions of eigenvalues and eigenfunctions of the Dirichlet problem for the Laplace operator was investigated in [20] and [21.

The case of general position for a 3-dimensional linear stationary system in the theory of elasticity was considered in 22] (see also [23]). For the oscillation of a membrane, see 24. Similar problems were considered in 33, 34, 35. In 45, the problem for a linear stationary system in the theory of elasticity, in domains with concentrated masses, was considered under the assumption that the oscillation laws for the body and the masses are different (the inclusions are stiffer). We also draw the reader's attention to the papers [36, 37, 38, 39, 40, 41, 42, 43, where the cases of second-order and fourth-order operators with various concentrated masses were considered.

The asymptotic behaviour of the oscillations of a body that has many small inclusions of high density situated periodically along the boundary was investigated in [25, 26, 27, 28, 29, 30, 31, 32. Questions about the limit (homogenized) behaviour of eigenvalues were studied. The boundary-value problem for a stationary system in the linear theory of elasticity with nonperiodic rapidly varying boundary conditions and a large number of concentrated masses near the boundary was investigated in [4]; its asymptotic behaviour was considered, as well as the limit behaviour of the spectrum of this boundary-value problem.

The case of a boundary-value problem for a system in the theory of elasticity where the limit problem has a boundary condition of the third kind on the boundary of the domain and the masses are light was examined. Estimates were obtained for the rate of convergence of a solution of the original problem to a solution of the homogenized one, as well as for the rate of convergence of eigenelements of this type of boundary-value problem.

The paper [46] is devoted to a detailed study of the behaviour of eigenelements of the Laplace operator in a domain with nonperiodic 'light' concentrated masses. A multidimensional problem in a domain with periodic sparsely situated 'light' masses was considered in 47. (see also 48). A homogenization theorem was proved and estimates were obtained for the deviation of the eigenelements of the original problem from the eigenelements of the homogenized one. Complete asymptotic expansions of eigenelements for the Laplace operator in domains with closely situated 'light' concentrated masses in the cases of two-dimensional and multi-dimensional domains were constructed in [49, 50, 51, 52. The cases of simple and of multiple eigenvalues of the limit problem were considered. Results which came from investigating problems with concentrated masses in the case of 'critical' density (with 'intermediate' masses) were announced in [53]. 
The problem in an unbounded domain with concentrated masses on the boundary was considered in [54]. It was proved that the poles of the analytic continuation of a solution of the original problem converge to the roots of eigenvalues of the limit problem in a bounded domain.

In this paper we consider a two-dimensional problem in a domain with periodically situated 'light' masses. By contrast with the papers [47, 48, 49, 50, 51, 52, it is assumed that the masses are situated on the boundary rather sparsely, as was assumed in [25, 26. 27, 28, 29, 30, 31, 32, when the distance between the masses is substantially greater than their diameter. The distance between the masses is assumed to be equal to $\varepsilon$, the diameter of the masses equal to $a \varepsilon$, where $a=a(\varepsilon) \rightarrow 0$ as $\varepsilon \rightarrow 0$, and the density is assumed to be equal to $\varepsilon^{-m}, m<2$. Furthermore, we assume that the limit boundary condition remains a Dirichlet condition, that is, $\varepsilon \ln a \rightarrow 0$ as $\varepsilon \rightarrow 0$ (a similar situation is investigated in [47] and [48]). In this paper, complete asymptotic expansions of eigenvalues and eigenfunctions are constructed by the method of matched asymptotic expansions [55, 56]. By contrast with the case of 'closely' situated masses (see [51]), it turns out that an intermediate layer of expansion appears in this problem. Then the substantiation of the asymptotics is carried out.

The paper consists of an Introduction and four sections. The problem is posed in $\S 1$, Convergence results are presented and estimates for solutions are expounded in $\S 2$. The third section is devoted to constructing the formal asymptotics of the eigenvalues and eigenfunctions. It consists of four subsections, each of which describes in detail the construction of terms for certain powers of the small parameters. In the first subsection, terms in powers of $\varepsilon$ are constructed, in the second terms in powers of $\ln a$, in the third terms in powers of $a$, and in the fourth terms in powers of $\mu=\varepsilon^{2-m} a^{2-m}$. The asymptotics we have constructed are rigorously substantiated in $\S 4$. In Appendix 1 the auxiliary results needed to construct the intermediate expansion are proved. Appendix 2 is devoted to proving auxiliary results of a qualitative nature for the construction of the inner expansion.

\section{The STATEMENT OF THE PROBLEM}

We begin with the construction of the domain. We use exactly the same notation and constructions as in [49, 50, 51. We denote by $\Omega$ a domain in $\mathbb{R}^{2}$ contained in the upper half-plane whose boundary is piecewise smooth and consists of several parts:

$$
\partial \Omega=\Gamma_{1} \cup \Gamma_{2} \cup \Gamma_{3} \cup \Gamma_{4}
$$

here $\Gamma_{4}=\Gamma_{\varepsilon} \cup \gamma_{\varepsilon}$, where $\Gamma_{4}$ is the interval $(-\pi / 2, \pi / 2)$ on the $x_{1}$-axis, while $\Gamma_{2}$ and $\Gamma_{3}$ belong to the straight lines $x_{1}=-\pi / 2$ and $x_{1}=\pi / 2$, respectively. Moreover, $\Gamma_{\varepsilon}$ and $\gamma_{\varepsilon}$ have micro-inhomogeneous structure and the small intervals that make them up alternate. Here $\varepsilon=1 /(2 \mathbf{N}+1)$ is a small parameter, $\mathbf{N}$ is a positive integer, $\mathbf{N} \gg 1$ (see Figure [1).

We now describe in more detail the fine structure of $\Gamma_{4}$. Let

$$
\begin{aligned}
& \gamma=\left\{\zeta:-1<\zeta_{1}<1, \zeta_{2}=0\right\} \\
& \Gamma=\left\{\zeta:-\infty<\zeta_{1}<-1,1<\zeta_{1}<+\infty, \zeta_{2}=0\right\}
\end{aligned}
$$

in the variables $\zeta=x /(a \varepsilon)$, where $0<a<\pi / 2$ and $a=a(\varepsilon) \rightarrow 0$ as $\varepsilon \rightarrow 0$. We set

$$
\gamma_{\varepsilon}=\left\{x \in \Gamma_{4}:\left(\frac{x_{1}}{a \varepsilon}-\frac{\mathbf{n} \pi}{\varepsilon}, 0\right) \in \gamma, \mathbf{n} \in \mathbb{Z}\right\}, \quad \Gamma_{\varepsilon}=\Gamma_{4} \backslash \gamma_{\varepsilon} .
$$




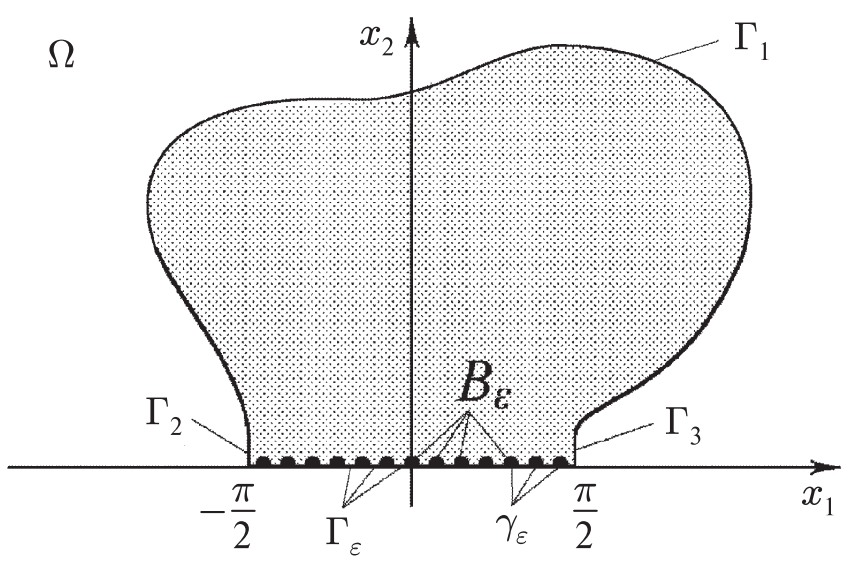

FiguRE 1. A membrane with a large number of concentrated masses on the boundary

We also use the following notation. Let

$$
\begin{aligned}
\Pi & =\left\{\xi:-\frac{\pi}{2}<\xi_{1}<\frac{\pi}{2}, \quad \xi_{2}>0\right\} \text { be a half-strip in the space } \xi=\frac{x}{\varepsilon}, \\
\Pi_{\xi_{2}^{0}} & =\left\{\xi:-\frac{\pi}{2}<\xi_{1}<\frac{\pi}{2}, \quad \xi_{2}>\xi_{2}^{0}\right\} \text { be a 'shortened' half-strip, } \\
\breve{\Sigma} & =\left\{\xi:-\frac{\pi}{2}<\xi_{1}<\frac{\pi}{2}, \quad \xi_{2}=0\right\}, \\
\Sigma & =\left\{\xi:-\frac{\pi}{2}<\xi_{1}<0, \quad 0<\xi_{1}<\frac{\pi}{2}, \quad \xi_{2}=0\right\},
\end{aligned}
$$

and let $B$ be the half-disc $\left\{\zeta: \zeta_{1}^{2}+\zeta_{2}^{2}<1, \zeta_{2}>0\right\}$ in the space $\zeta=\xi / a=x /(a \varepsilon)$ (see Figures 2 and 3 ).

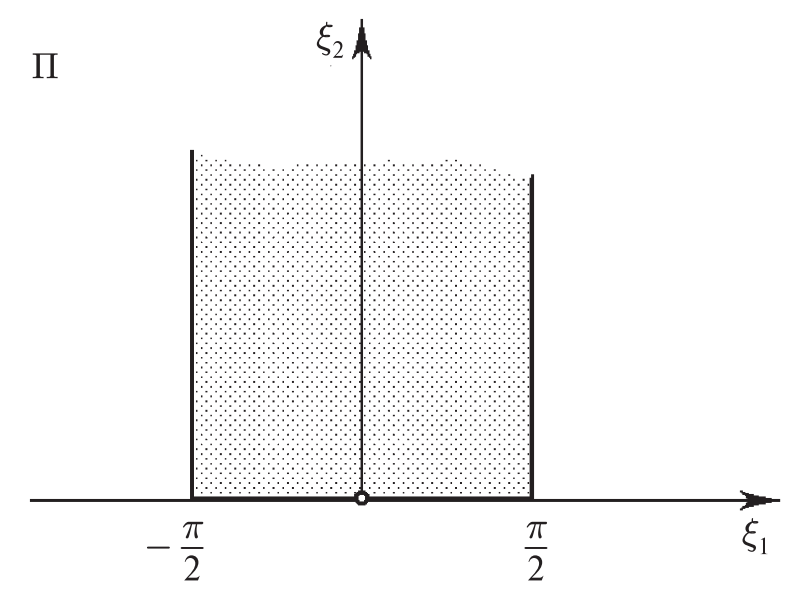

FiguRE 2. A periodicity cell in the intermediate expansion

We set

$$
B_{\varepsilon}^{\mathbf{n}}=\left\{x \in \Omega:\left(\frac{x_{1}}{a \varepsilon}-\frac{\mathbf{n} \pi}{\varepsilon}, \frac{x_{2}}{a \varepsilon}\right) \in B\right\}, \quad \mathbf{n} \in \mathbb{Z}, \quad B_{\varepsilon}=\bigcup B_{\varepsilon}^{\mathbf{n}},
$$


and, correspondingly,

$$
\gamma_{\varepsilon}^{\mathbf{n}}=\left\{x \in \Gamma_{4}:\left(\frac{x_{1}}{a \varepsilon}-\frac{\mathbf{n} \pi}{\varepsilon}, 0\right) \in \gamma\right\}, \quad \mathbf{n} \in \mathbb{Z},
$$

that is,

$$
\gamma_{\varepsilon}=\bigcup \gamma_{\varepsilon}^{\mathbf{n}}
$$

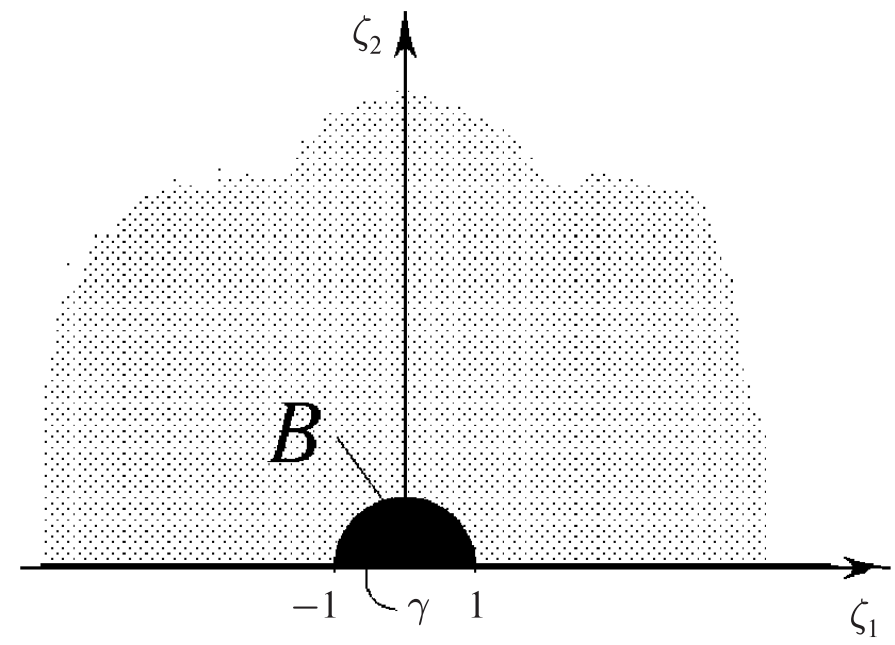

Figure 3. A cell in the inner expansion

We assume that $a(\varepsilon)$ is a function of $\varepsilon$ such that $a(\varepsilon) \rightarrow 0$ as $\varepsilon \rightarrow 0$ and

$$
\lim _{\varepsilon \rightarrow 0} \varepsilon \ln a=0 .
$$

Our aim is to construct the asymptotics as $\varepsilon \rightarrow 0$ of the eigenelements of the following spectral problem:

$$
\begin{cases}-\Delta u_{\varepsilon}=\lambda_{\varepsilon} \rho_{\varepsilon} u_{\varepsilon} & \text { for } x \in \Omega, \\ u_{\varepsilon}=0 & \text { for } x \in \gamma_{\varepsilon}, \\ \frac{\partial u_{\varepsilon}}{\partial \nu}=0 & \text { for } x \in \Gamma_{\varepsilon} \cup \Gamma_{1} \cup \Gamma_{2} \cup \Gamma_{3},\end{cases}
$$

where $\rho_{\varepsilon}(x)$ is a density that has the form

$$
\rho_{\varepsilon}(x)= \begin{cases}1 & \text { in } \Omega \backslash \bar{B}_{\varepsilon}, \\ 1+(a \varepsilon)^{-m} & \text { in } B_{\varepsilon} .\end{cases}
$$

Throughout what follows we take $m$ to be a constant, $0<m<2$. We call the sets $B_{\varepsilon}^{\mathbf{n}}$ concentrated masses. We also consider the problem

$$
\begin{cases}-\Delta u_{0}=\lambda u_{0} & \text { for } x \in \Omega, \\ u_{0}=0 & \text { for } x \in \Gamma_{4}, \\ \frac{\partial u_{0}}{\partial \nu}=0 & \text { for } x \in \Gamma_{1} \cup \Gamma_{2} \cup \Gamma_{3} .\end{cases}
$$

In the following section we shall explain why problem (4) is the limit problem for problem (2) (in the absence of masses, see the proof in [57, as well as in 58] ). Condition (11) guarantees that there is a homogeneous Dirichlet condition on $\Gamma_{4}$. 


\section{Convergence And estimates}

We consider the boundary-value problem

$$
\begin{cases}-\Delta U_{\varepsilon}=\lambda \rho_{\varepsilon} U_{\varepsilon}+f & \text { for } x \in \Omega, \\ U_{\varepsilon}=0 & \text { for } x \in \gamma_{\varepsilon}, \\ \frac{\partial U_{\varepsilon}}{\partial \nu}=0 & \text { for } x \in \Gamma_{\varepsilon} \cup \Gamma_{1} \cup \Gamma_{2} \cup \Gamma_{3},\end{cases}
$$

where $\rho_{\varepsilon}(x)$ is a density that has the form (3), and the problem

$$
\left\{\begin{array}{l}
-\Delta U_{0}=\lambda U_{0}+f \quad \text { for } x \in \Omega, \\
U_{0}=0 \text { for } x \in \Gamma_{4}, \\
\frac{\partial U_{0}}{\partial \nu}=0 \quad \text { for } x \in \Gamma_{1} \cup \Gamma_{2} \cup \Gamma_{3},
\end{array}\right.
$$

which is called the limit (homogenized) problem. We understand a solution of (2), (4), (5), and (6) to mean a solution in the generalized sense [59] (see also [60, 61, 62]). Let $f \in L_{2}(\Omega)$. We denote by $\|u\|_{0}$ and $\|u\|_{1}$ the norms of the function $u$ in the spaces $L_{2}(\Omega)$ and $H^{1}(\Omega)$, respectively. Now, we present the following three theorems.

Theorem 2.1. Let $f \in L_{2}(\Omega)$ and let $K$ be an arbitrary compact set in the complex plane $\mathbb{C}$ that does not contain eigenvalues of the limit problem (4). Then

1) there is a number $\varepsilon_{0}>0$ such that for any $\varepsilon<\varepsilon_{0}$ and any $\lambda \in K$ there exists a unique solution of the problem (5) and the following estimate, uniform with respect to $\varepsilon$ and $\lambda$, holds:

$$
\left\|U_{\varepsilon}\right\|_{1} \leq C\|f\|_{0},
$$

where $C$ is also independent of $f$;

2) solutions of problems (5) and (6) satisfy

$$
\left\|U_{\varepsilon}-U_{0}\right\|_{1} \longrightarrow 0 \quad \text { as } \varepsilon \rightarrow 0 .
$$

Theorem 2.2. Let $\lambda_{0}$ be an eigenvalue of the limit problem (4). Then

1) there exists an eigenvalue $\lambda_{\varepsilon}$ of the original problem (2) converging to $\lambda_{0}$ as $\varepsilon \rightarrow 0$

2) if $\lambda_{0}$ has multiplicity $N$, then there are $N$ eigenvalues of the original problem (accounting for the total multiplicity) converging to $\lambda_{0}$.

Theorem 2.3. Let $f \in L_{2}(\Omega)$. If an eigenvalue $\lambda_{0}$ of the limit problem (4) has multiplicity $N$, then

1) for any $\lambda$ close to $\lambda_{0}$, the solution $U_{\varepsilon}$ of the boundary-value problem (5) satisfies the following uniform estimate:

$$
\left\|U_{\varepsilon}\right\|_{1} \leq C \frac{\|f\|_{0}}{\prod_{j=1}^{N}\left|\lambda_{\varepsilon}^{j}-\lambda\right|},
$$

where $\lambda_{\varepsilon}^{1}, \ldots, \lambda_{\varepsilon}^{N}$ are eigenvalues of problem (2) converging to $\lambda_{0}$.

2 ) if the solution $U_{\varepsilon}$ of problem (5) is orthogonal in $L_{2}(\Omega)$ to an eigenfunction $u_{\varepsilon}^{i}$ of problem (2) corresponding to $\lambda_{\varepsilon}^{i}$, then it satisfies

$$
\left\|U_{\varepsilon}\right\|_{1} \leq C \frac{\|f\|_{0}}{\prod_{j=1 ; j \neq i}^{N}\left|\lambda_{\varepsilon}^{j}-\lambda\right|} .
$$


The proofs of these theorems are based on the scheme in [63; see also 64, 65, 66, 67, and [58. However, the presence of concentrated masses sparsely situated on the boundary means we have to modify this scheme. A modification was made in [51] for the case of 'frequent' masses. The proofs of the theorems stated above repeat the proofs in [51] almost word for word. The only difference is a change in the proof of Lemma 2.2 in [51]. For the problem with 'sparse' masses, the proof of Lemma 2.2 follows from results in [57. In what follows, Theorem 2.3 will be used to estimate the discrepancy and substantiate the formal asymptotic expansion.

\section{FORMAL ASYMPTOTIC ANALYSIS}

To construct the asymptotics of the eigenvalues and eigenfunctions we use the method of matched asymptotic expansions (see [55, 68, 69, 70], as well as [56, 71, 72]).

3.1. The construction of the asymptotics. Step I. We consider the case of a simple eigenvalue; that is, we assume that in the spectral problem (4), $\lambda_{0}$ is simple, and for definiteness we assume that $u_{0}(x)$ is normalized in $L_{2}(\Omega)$. Since (44) is the limit problem for problem (2) and $\lambda_{0}$ and $u_{0}$ are eigenelements of problem (4), it is natural to begin by looking for an expansion of a solution to problem (2) in the form

$$
u_{\varepsilon}(x)=u_{0}(x)+\sum_{j=1}^{+\infty} \varepsilon^{j} u_{j}(x)+\cdots,
$$

and an expansion of the eigenvalues in the form

$$
\lambda_{\varepsilon}=\lambda_{0}+\sum_{j=1}^{+\infty} \varepsilon^{j} \lambda_{j}+\cdots
$$

We note here that the function $u_{0}(x)$ belongs to $C^{\infty}(\bar{\Omega})$; for the remaining coefficients $u_{j}(x)$ in (11) we seek solutions in $C^{\infty}(\bar{\Omega})$ to

$$
\left\{\begin{array}{rlrl}
-\Delta u_{j} & =\lambda_{0} u_{j}+\lambda_{1} u_{j-1}+\cdots+\lambda_{j} u_{0} & & \text { for } x \in \Omega, \\
\frac{\partial u_{j}}{\partial \nu} & =0 \quad \text { for } x \in \Gamma_{1} \cup \Gamma_{2} \cup \Gamma_{3}, & & j=1,2, \ldots, \\
u_{j} & =\alpha_{j}^{0}\left(x_{1}\right) \quad \text { for } x \in \Gamma_{4}, &
\end{array}\right.
$$

where the $\alpha_{j}^{0}\left(x_{1}\right)$ are, at present, unknown (arbitrary) functions whose odd derivatives vanish at $x_{1}= \pm \pi / 2$.

So that the problems (13) are uniquely soluble, henceforth we will choose all the solutions to be orthogonal to $u_{0}$ in $L_{2}(\Omega)$.

Remark 3.1. The equations in (13) and the boundary conditions outside $\Gamma_{4}$ are obtained by substituting the series (11) and the expansion (12) into (22) and formally equating the coefficients of terms with like powers of $\varepsilon$. We use the outer expansion (11) outside some small neighbourhood of $\Gamma_{4}$; therefore at present we take the boundary conditions on $\Gamma_{4}$ to be arbitrary and use Dirichlet boundary conditions for convenience. The requirement

that the odd derivatives of the $\alpha_{j}^{0}$ vanish is a necessary condition for (13) to have a solution in the class $C^{\infty}(\bar{\Omega})$.

In a small neighbourhood of $\Gamma_{4}$, we construct other series ('intermediate' and 'inner' expansions). To do this, we expand the functions $u_{j}(x)$ as Taylor series in the variable $x_{2}$ 
as $x_{2} \rightarrow 0$. Since the functions $u_{k}$ are infinitely differentiable, we have

$$
\begin{aligned}
& u_{0}(x)=\alpha_{0}^{1}\left(x_{1}\right) x_{2}+\sum_{l=2}^{+\infty} \alpha_{0}^{l}\left(x_{1}\right) x_{2}^{l}, \\
& u_{j}(x)=\alpha_{j}^{0}\left(x_{1}\right)+\alpha_{j}^{1}\left(x_{1}\right) x_{2}+\sum_{l=2}^{+\infty} \alpha_{j}^{l}\left(x_{1}\right) x_{2}^{l}, \quad j=1, \ldots,
\end{aligned}
$$

where

$$
\alpha_{k}^{l}=\left.\frac{1}{(l) !} \frac{\partial^{l} u_{k}}{\partial x_{2}^{l}}\right|_{x_{2}=0}, \quad k=0,1, \ldots,
$$

and using the Neumann boundary conditions on $\Gamma_{2} \cup \Gamma_{3}$, the equation (13) and infinite differentiability, we have

$$
\frac{d^{2 j+1} \alpha_{k}^{l}}{d x_{1}^{2 j+1}}\left( \pm \frac{\pi}{2}\right)=0, \quad l=1, \ldots, \quad j=0, \ldots
$$

We emphasize that all the $\alpha_{0}^{l}\left(x_{1}\right)$ are known functions (since $u_{0}(x)$ is the solution of (4)) and by the equation in (4) we have

$$
\alpha_{0}^{2 l}\left(x_{1}\right) \equiv 0, \quad l=0,1, \ldots .
$$

We also note that condition (14) holds for the known functions $\alpha_{0}^{l}$. For now, it is a condition that the functions $\alpha_{k}^{0}$ have to satisfy. We will check that it is satisfied in what follows (then condition (14) will hold automatically for the other functions $\alpha_{k}^{l}$ due to the boundary-value problems (13)).

We make the change of variables $\xi_{2}=x_{2} / \varepsilon$ (that is, we rewrite these expansions in terms of the 'intermediate' variables). Then as $\varepsilon \xi_{2} \rightarrow 0$ we have

$$
\begin{aligned}
u_{0}\left(x_{1}, \varepsilon \xi_{2}\right) & =\varepsilon \alpha_{0}^{1}\left(x_{1}\right) \xi_{2}+\sum_{l=1}^{+\infty} \varepsilon^{2 l+1} \alpha_{0}^{2 l+1}\left(x_{1}\right) \xi_{2}^{2 l+1}, \\
\varepsilon^{j} u_{j}\left(x_{1}, \varepsilon \xi_{2}\right) & =\varepsilon^{j} \alpha_{j}^{0}\left(x_{1}\right)+\varepsilon^{j+1} \alpha_{j}^{1}\left(x_{1}\right) \xi_{2}+\sum_{l=2}^{+\infty} \varepsilon^{j+l} \alpha_{j}^{l}\left(x_{1}\right) \xi_{2}^{l}, \quad j \in \mathbb{N} .
\end{aligned}
$$

Substituting (15) into (11) we see that (11) has the following form as $\varepsilon \xi_{2} \rightarrow 0$ :

$$
u_{\varepsilon}(x)=\sum_{j=0}^{+\infty} \varepsilon^{j} u_{j}\left(x_{1}, \varepsilon \xi_{2}\right)+\cdots=\sum_{j=0}^{+\infty} \varepsilon^{j} V_{j}\left(\xi_{2} ; x_{1}\right)+\cdots,
$$

where

$$
\begin{aligned}
V_{2 j+1}\left(\xi_{2} ; x_{1}\right) & =\alpha_{0}^{2 j+1}\left(x_{1}\right) \xi_{2}^{2 j+1}+\alpha_{1}^{2 j}\left(x_{1}\right) \xi_{2}^{2 j}+\cdots+\alpha_{2 j+1}^{0}\left(x_{1}\right), \\
V_{2 j}\left(\xi_{2} ; x_{1}\right) & =\alpha_{1}^{2 j-1}\left(x_{1}\right) \xi_{2}^{2 j-1}+\cdots+\alpha_{2 j}^{0}\left(x_{1}\right), \quad j=0,1, \ldots,
\end{aligned}
$$

and $\xi=x / \varepsilon$.

Following the method of matched asymptotic expansions, we conclude that the intermediate expansion must have the structure

$$
u_{\varepsilon}(x)=\varepsilon v_{1}\left(\frac{x}{\varepsilon} ; x_{1}\right)+\sum_{j=2}^{+\infty} \varepsilon^{j} v_{j}\left(\frac{x}{\varepsilon} ; x_{1}\right)+\cdots,
$$

where

$$
v_{q}\left(\xi ; x_{1}\right)=V_{q}\left(\xi_{2} ; x_{1}\right)+o(1) \quad \text { as } \xi_{2} \rightarrow+\infty \text {. }
$$


Here $x_{1}$ is a 'slow' variable; sometimes the dependence on $x_{1}$ will be omitted from the arguments. In terms of the variables $\left(\xi ; x_{1}\right)$ the equation in (2) becomes

$$
-\varepsilon^{-2} \Delta_{\xi} u_{\varepsilon}-2 \varepsilon^{-1} \frac{\partial^{2} u_{\varepsilon}}{\partial x_{1} \partial \xi_{1}}-\frac{\partial^{2} u_{\varepsilon}}{\partial x_{1}^{2}}=\lambda_{\varepsilon} \rho_{\varepsilon} u_{\varepsilon}
$$

while the boundary conditions on $\Gamma_{2}$ take the form

$$
\frac{\partial u_{\varepsilon}}{\partial \nu}=-\varepsilon^{-1} \frac{\partial u_{\varepsilon}}{\partial \xi_{1}}-\frac{\partial u_{\varepsilon}}{\partial x_{1}}=0
$$

and on $\Gamma_{3}$,

$$
\frac{\partial u_{\varepsilon}}{\partial \nu}=\varepsilon^{-1} \frac{\partial u_{\varepsilon}}{\partial \xi_{1}}+\frac{\partial u_{\varepsilon}}{\partial x_{1}}=0
$$

Remark 3.2. In what follows we construct terms of the 'intermediate' expansion (18) in the form of functions that are $\pi$-periodic in $\xi_{1}$ (for each fixed value of $x_{1}$ ).

Rewriting the equation and the boundary conditions in the coordinates $\xi$ (see (20), (21), and (22)), substituting the 'intermediate' expansion (18) and the series (12) into problem (2), we collect terms with like powers of $\varepsilon$ (terms in $\varepsilon^{q-1}$ in the equation and terms in $\varepsilon^{q}$ in the boundary conditions). Passing to the limit as $a \rightarrow 0$ in the cell $\Pi$, taking account of (19), (17) and Remark 3.2, we obtain the following problems: for $v_{1}$,

$$
\left\{\begin{array}{l}
-\Delta_{\xi} v_{1}=0 \quad \text { in } \Pi, \\
\frac{\partial v_{1}}{\partial \xi_{2}}=0 \quad \text { on } \Sigma \\
\frac{\partial v_{1}}{\partial \xi_{1}}\left(\xi ; \pm \frac{\pi}{2}\right)=0 \quad \text { at } \xi_{1}= \pm \frac{\pi}{2} \\
v_{1} \sim \alpha_{0}^{1} \xi_{2}+\alpha_{1}^{0} \quad \text { as } \xi_{2} \rightarrow+\infty
\end{array}\right.
$$

and for the $v_{j}$ for $j>1$,

$$
\left\{\begin{array}{l}
-\Delta_{\xi} v_{j}=2 \frac{\partial^{2} v_{j-1}}{\partial x_{1} \partial \xi_{1}}+\frac{\partial^{2} v_{j-2}}{\partial x_{1}^{2}}+\sum_{k=0}^{j-2} \lambda_{k} v_{j-2-k} \quad \text { in } \Pi, \\
\frac{\partial v_{j}}{\partial \xi_{2}}=0 \quad \text { on } \Sigma \\
\frac{\partial v_{j}}{\partial \xi_{1}}\left(\xi ; \pm \frac{\pi}{2}\right)=-\frac{\partial v_{j-1}}{\partial x_{1}}\left(\xi ; \pm \frac{\pi}{2}\right) \quad \text { at } \xi_{1}= \pm \frac{\pi}{2} \\
v_{j}=V_{j}+o(1) \quad \text { as } \xi_{2} \rightarrow+\infty
\end{array}\right.
$$

From now on, we set functions with indices we have not introduced earlier to be equal to zero; that is, for example, $v_{-1} \equiv v_{0} \equiv 0$.

We emphasize that from the boundary-value problems (13) and the definition of $V_{q}$, we have the following equalities:

$$
\begin{gathered}
-\Delta_{\xi} V_{q}=2 \frac{\partial^{2} V_{q-1}}{\partial x_{1} \partial \xi_{1}}+\frac{\partial^{2} V_{q-2}}{\partial x_{1}^{2}}+\lambda_{0} V_{q-2}+\cdots+\lambda_{q-3} V_{1}, \\
\frac{\partial^{2 n+1} V_{q}}{\partial x_{1}^{2 n+1}}\left(\xi_{2} ; \pm \frac{\pi}{2}\right)=0, \quad \frac{\partial V_{q}}{\partial \xi_{1}}\left(\xi_{2} ; x_{1}\right) \equiv 0 .
\end{gathered}
$$


We introduce into consideration the auxiliary problem

$$
\left\{\begin{aligned}
-\Delta_{\xi} X & =0 \quad & & \text { in } \Pi, \\
\frac{\partial X}{\partial \xi_{2}} & =0 & & \text { on } \Sigma \\
\frac{\partial X}{\partial \xi_{1}} & =0 & & \text { at } \xi_{1}= \pm \frac{\pi}{2} \\
X & \sim \xi_{2} & & \text { as } \xi_{2} \rightarrow+\infty .
\end{aligned}\right.
$$

We also consider the following function (see [71], as well as [72] and [56]):

$$
X(\xi)=\operatorname{Re} \ln \sin z+\ln 2,
$$

where $z=\xi_{1}+\mathrm{i} \xi_{2}$. It follows from (28) that the function

$$
X(\xi) \in C^{\infty}\left(\overline{\mathbb{R}_{+}^{2}} \backslash \bigcup_{k \in \mathbb{Z}}\{(k \pi, 0)\}\right)
$$

is $\pi$-periodic in $\xi_{1}$; the asymptotics of the function $X$ at infinity as $\xi_{2} \rightarrow+\infty$ have the form:

$$
X(\xi)=\xi_{2}+O\left(e^{-2 \xi_{2}}\right),
$$

while the asymptotics of this function at zero $($ as $\rho \equiv|\xi| \rightarrow 0)$ are

$$
X(\xi)=\ln \rho+\ln 2+\sum_{j=1}^{+\infty} B_{2 j} \rho^{2 j} \cos 2 j \theta,
$$

where the $B_{2 j}$ are constants that can be calculated explicitly. It is easy to verify that $X$ is a solution of (27). Then obviously

$$
v_{1}\left(\xi ; x_{1}\right)=\alpha_{0}^{1}\left(x_{1}\right) X(\xi)+\alpha_{1}^{0}\left(x_{1}\right)
$$

is a solution of problem (23), where $\alpha_{1}^{0}\left(x_{1}\right) \in C^{\infty}[-\pi / 2, \pi / 2]$ is at present arbitrary (a boundary condition for $u_{1}(x)$ ), which satisfies (14). This function is $\pi$-periodic and even in $\xi_{1}$. By (28) and (31), as $\xi_{2} \rightarrow+\infty$, the asymptotics of the function $v_{1}\left(\xi ; x_{1}\right)$ at infinity are as follows:

$$
v_{1}\left(\xi ; x_{1}\right)=\alpha_{1}^{0}\left(x_{1}\right)+\alpha_{0}^{1}\left(x_{1}\right)\left[\xi_{2}+O\left(e^{-2 \xi_{2}}\right)\right] .
$$

In view of (30) and (31), the asymptotics of this function at zero (as $\rho \rightarrow 0$ ) take the form:

$$
v_{1}\left(\xi ; x_{1}\right)=\alpha_{0}^{1}\left(x_{1}\right)(\ln \rho+\ln 2)+\alpha_{1}^{0}\left(x_{1}\right)+O\left(\rho^{2}\right) .
$$

We also seek the other terms of the intermediate expansion such that

$$
v_{q}=O(\ln \rho) \quad \text { as } \quad \rho \rightarrow 0 .
$$

We note that according to (14) we have

$$
\frac{\partial v_{1}}{\partial x_{1}}\left(\xi ; x_{1}\right)=0 \quad \text { at } \quad x_{1}= \pm \frac{\pi}{2}
$$

and, consequently, by (21), (22), (23) and (35), we have

$$
\frac{\partial v_{1}}{\partial \nu}\left(\frac{x}{\varepsilon} ; x_{1}\right)=0 \quad \text { on } \quad \Gamma_{2} \cup \Gamma_{3} .
$$

The function $v_{1}$ does not satisfy the Dirichlet boundary condition on $\gamma_{\varepsilon}$; therefore we introduce yet another ('inner') expansion, going over to the variables

$$
\zeta^{\mathbf{n}} \equiv\left(\zeta_{1}^{\mathbf{n}}, \zeta_{2}^{\mathbf{n}}\right)=\left(\frac{\xi_{1}}{a}-\frac{\mathbf{n} \pi}{\varepsilon}, \frac{\xi_{2}}{a}\right), \quad \mathbf{n} \in \mathbb{Z} .
$$


We rewrite the asymptotics of $\varepsilon v_{1}\left(\xi ; x_{1}\right)$ as $\rho \rightarrow 0$ in terms of the variables $\zeta^{\mathbf{n}}$ (more precisely, in the polar coordinates $\left(\tau^{\mathbf{n}}, \theta^{\mathbf{n}}\right)$ for $\left.\zeta^{\mathbf{n}}\right)$, taking (33) into account. As $a \tau^{\mathbf{n}} \rightarrow 0$, we have

$$
\varepsilon v_{1}\left(a \zeta^{\mathbf{n}} ; x_{1}\right)=\varepsilon \alpha_{0}^{1}\left(x_{1}\right)\left[\ln a+\left(\ln \tau^{\mathbf{n}}+\ln 2\right)\right]+\varepsilon \alpha_{1}^{0}\left(x_{1}\right)+O\left(\varepsilon a^{2}\left(\tau^{\mathbf{n}}\right)^{2}\right) .
$$

Rewriting the asymptotics of the series (18) as $\rho \rightarrow 0$ in terms of $\zeta^{\mathbf{n}}$, taking account of (37) and (34), and following the method of matched asymptotic expansions, we conclude that the expansion of the function $u_{\varepsilon}$ in the new variables $\zeta^{\mathbf{n}}$ must have the form

$$
u_{\varepsilon}(x)=\varepsilon \ln a w_{1,1,0}^{\mathbf{n}}\left(\zeta^{\mathbf{n}} ; x_{1}\right)+\varepsilon w_{1,0,0}^{\mathbf{n}}\left(\zeta^{\mathbf{n}} ; x_{1}\right)+\cdots,
$$

where, as $\tau^{\mathbf{n}} \rightarrow+\infty$, the term $w_{1,0,0}^{\mathbf{n}}$ has the asymptotic form

$$
w_{1,0,0}^{\mathbf{n}} \sim \alpha_{0}^{1}\left(\ln \tau^{\mathbf{n}}+\ln 2\right)+\alpha_{1}^{0} .
$$

For brevity we omit the index $\mathbf{n}$ from the functions $w$ and variables $\zeta$ in what follows. Then in the variables $\left(\zeta ; x_{1}\right)$ the equation in problem (2) takes the form

$$
-\varepsilon^{-2} a^{-2} \Delta_{\zeta} u_{\varepsilon}-2 \varepsilon^{-1} a^{-1} \frac{\partial^{2} u_{\varepsilon}}{\partial x_{1} \partial \zeta_{1}}-\frac{\partial^{2} u_{\varepsilon}}{\partial x_{1}^{2}}=\lambda_{\varepsilon} \rho_{\varepsilon} u_{\varepsilon} .
$$

Taking the form of equation (40) in the inner variables $\zeta$ into account, we substitute (38) and the series (12) into (2) and collect terms with like powers of $\varepsilon, a$, and $\ln a$ (in the equation with $\varepsilon^{-1} a^{-2}$, and in the boundary conditions with $a^{-1}$, respectively). In view of (39) we obtain a problem for the function $w_{1,0,0}$ of the form

$$
\left\{\begin{aligned}
\Delta_{\zeta} w_{1,0,0} & =0 \quad \text { in } \mathbb{R}_{+}^{2}, \\
\frac{\partial w_{1,0,0}}{\partial \zeta_{2}} & =0 \quad \text { on } \Gamma \\
w_{1,0,0} & =0 \quad \text { on } \gamma \\
w_{1,0,0} & \sim \alpha_{0}^{1}(\ln \tau+\ln 2)+\alpha_{1}^{0} \quad \text { as } \tau \rightarrow+\infty
\end{aligned}\right.
$$

Recall that

$\gamma=\left\{\zeta:-1<\zeta_{1}<1, \zeta_{2}=0\right\}$ and $\Gamma=\left\{\zeta:-\infty<\zeta_{1}<-1,1<\zeta_{1}<+\infty, \zeta_{2}=0\right\}$.

Consider the following function $Y$ (see [73]):

$$
Y(\zeta)=\operatorname{Re} \ln \left(y+\sqrt{y^{2}-1}\right)
$$

where $y=\zeta_{1}+\mathrm{i} \zeta_{2}$. It is easy to verify that $Y \in H_{\mathrm{loc}}^{1}\left(\mathbb{R}_{+}^{2}\right)$ is a solution of the problem

$$
\left\{\begin{aligned}
\Delta_{\zeta} Y=0 & \text { in } \mathbb{R}_{+}^{2}, \\
\frac{\partial Y}{\partial \zeta_{2}}=0 & \text { on } \Gamma, \\
Y=0 & \text { on } \gamma .
\end{aligned}\right.
$$

It follows from the explicit form of the function $Y$ that, as $\tau \rightarrow+\infty$, it has the asymptotic form

$$
Y(\zeta)=(\ln \tau+\ln 2)+O\left(\tau^{-1}\right) .
$$

Taking (44) and problem (43) into account, we conclude that the function

$$
w_{1,0,0}\left(\zeta ; x_{1}\right)=\alpha_{0}^{1}\left(x_{1}\right) Y(\zeta)
$$

is a solution of problem (41) for

$$
\alpha_{1}^{0}\left(x_{1}\right) \equiv 0
$$


G. A. CHECHKIN

Therefore we take $w_{1,0,0}$ and $\alpha_{1}^{0}$ to be given by (45) and (46). Then by (31) the function $v_{1}$ is determined by the equality

$$
v_{1}\left(\xi ; x_{1}\right)=\alpha_{0}^{1}\left(x_{1}\right) X(\xi)
$$

and (13), the problem for $u_{1}$, takes the form

$$
\left\{\begin{aligned}
-\Delta u_{1} & =\lambda_{0} u_{1}+\lambda_{1} u_{0} \quad \text { for } x \in \Omega, \\
u_{1} & =0 \quad \text { for } x \in \Gamma_{4}, \\
\frac{\partial u_{1}}{\partial \nu} & =0 \quad \text { for } x \in \Gamma_{1} \cup \Gamma_{2} \cup \Gamma_{3} .
\end{aligned}\right.
$$

Obviously, the pair

$$
u_{1} \equiv 0, \quad \lambda_{1}=0
$$

satisfies (48). Thus, it is clear that

$$
\alpha_{1}^{l} \equiv 0 .
$$

Remark 3.3. Note that the arbitrary $\alpha_{1}^{0}\left(x_{1}\right)$ is fixed for the function $v_{1}\left(\xi ; x_{1}\right)$ by the matching condition for the functions $v_{1}\left(\xi ; x_{1}\right)$ and $w_{1,0,0}\left(\zeta ; x_{1}\right)$, and this is how $v_{1}\left(\xi ; x_{1}\right)$ is finally determined. We also emphasize that in what follows the arbitrary summands independent of $\xi$, appearing in the terms

$$
\varepsilon^{j+p(m-2)} a^{k+p(m-2)} \ln ^{l} a v_{j, k, l, p}\left(\xi ; x_{1}\right)
$$

in the intermediate expansion, are determined from the matching condition with the terms of the inner expansion

$$
\varepsilon^{j+p(m-2)} a^{k+p(m-2)} \ln ^{l} a w_{j, k, l, p}\left(\zeta ; x_{1}\right)
$$

(for the same exponents $j, k, l$ ).

We continue our investigation of problem (24). We write out the problem for $j=2$, taking (17), (35) and (50) into account:

$$
\left\{\begin{aligned}
-\Delta_{\xi} v_{2} & =2 \frac{\partial^{2} v_{1}}{\partial x_{1} \partial \xi_{1}} \quad \text { in } \Pi, \\
\frac{\partial v_{2}}{\partial \xi_{2}} & =0 \quad \text { on } \Sigma \\
\frac{\partial v_{2}}{\partial \xi_{1}}\left(\xi ; \pm \frac{\pi}{2}\right) & =0 \quad \text { at } \xi_{1}= \pm \frac{\pi}{2}, \\
v_{2} & \sim \alpha_{2}^{0} \quad \text { as } \xi_{2} \rightarrow+\infty
\end{aligned}\right.
$$

where, up to now, the function $\alpha_{2}^{0}\left(x_{1}\right) \in C^{\infty}[-\pi / 2, \pi / 2]$ is an arbitrary summand (a boundary condition for $u_{2}(x)$ ) that satisfies (14).

We consider the auxiliary problem

$$
\left\{\begin{aligned}
-\Delta_{\xi} X_{2} & =2 \frac{\partial X}{\partial \xi_{1}} \quad \text { in } \Pi, \\
\frac{\partial X_{2}}{\partial \xi_{2}} & =0 \quad \text { on } \Sigma, \\
X_{2} & =0 \quad \text { at } \xi_{1}= \pm \frac{\pi}{2}, \\
X_{2} & =o(1) \quad \text { as } \xi_{2} \rightarrow+\infty .
\end{aligned}\right.
$$


We introduce an auxiliary smooth cutoff function $0<\chi(\rho)<1$ such that

$$
\chi(\rho)= \begin{cases}1, & \rho>\frac{\pi}{4}, \\ 0, & \rho<\frac{\pi}{8} .\end{cases}
$$

Lemma 3.1. There exists a solution of (52) that is $\pi$-periodic and odd in $\xi_{1}$ and is such that

$$
X_{2} \in C^{\infty}\left(\overline{\mathbb{R}_{+}^{2}} \backslash \bigcup_{k \in \mathbb{Z}}\{(k \pi, 0)\}\right), \quad e^{\varpi \xi_{2}} \chi(\rho) X_{2} \in H^{1}(\Pi), \quad 0<\varpi<2,
$$

and the asymptotic behaviour

$$
\begin{aligned}
X_{2}(\xi)= & Q \rho \ln \rho \cos \theta+\sum_{j=0}^{\infty} \beta_{2 j+1} \rho^{2 j+3} \cos (2 j+1) \theta \\
& +\sum_{j=0}^{\infty} \widetilde{\beta}_{2 j+1} \rho^{2 j+1} \cos (2 j+1) \theta
\end{aligned}
$$

holds as $\rho \rightarrow 0$, where $Q, \beta_{k}$, and $\widetilde{\beta}_{k}$ are some constants.

The proof of the lemma is given in Appendix 1 (\$4).

By Lemma 3.1, (31), (52) and (14), the function

$$
v_{2}\left(\xi ; x_{1}\right)=\left(\alpha_{0}^{1}\right)^{\prime}\left(x_{1}\right) X_{2}(\xi)+\alpha_{2}^{0}\left(x_{1}\right)
$$

is a solution of (51) with the asymptotic behaviour

$$
v_{2}\left(\xi ; x_{1}\right)=\alpha_{2}^{0}\left(x_{1}\right)+O(\rho \ln \rho) \quad \text { as } \quad \rho \rightarrow 0
$$

and

$$
v_{2}=\alpha_{2}^{0}\left(x_{1}\right)+\widehat{v}_{2}\left(\xi ; x_{1}\right), \quad\left\|\widehat{v}_{2}\right\|_{H^{1}\left(\Pi_{\xi_{2}}\right)} \leq C e^{-\varpi \xi_{2}} \quad \text { as } \quad \xi_{2} \rightarrow+\infty,
$$

at infinity, where the constant $C$ is independent of $x_{1}$. Recall that

$$
\Pi_{b}=\left\{\xi:-\frac{\pi}{2}<\xi_{1}<\frac{\pi}{2}, \xi_{2}>b\right\} .
$$

Note that because of (14) and the boundary conditions in (52) we have

$$
\frac{\partial v_{2}}{\partial x_{1}}\left(\xi ; x_{1}\right)=0 \quad \text { at } \quad x_{1}= \pm \frac{\pi}{2}
$$

and, consequently, by (21), (22), (51) and (57),

$$
\frac{\partial v_{2}}{\partial \nu}\left(\frac{x}{\varepsilon} ; x_{1}\right)=0 \quad \text { on } \quad \Gamma_{2} \cup \Gamma_{3} .
$$

Rewriting (55) in terms of $\zeta$ :

$$
v_{2}\left(\xi ; x_{1}\right)=\alpha_{2}^{0}\left(x_{1}\right)+O(a \tau \ln (a \tau)) \quad \text { as } \quad a \tau \rightarrow 0,
$$

and then rewriting the asymptotics of the series (18) as $\rho \rightarrow 0$ in terms of the variables $\zeta$, taking (59) and (34) into account, and following the method of matched asymptotic expansions, we see that a new term must be introduced into the expansion (38):

$$
u_{\varepsilon}(x)=\varepsilon \ln a w_{1,1,0}\left(\zeta ; x_{1}\right)+\varepsilon w_{1,0,0}\left(\zeta ; x_{1}\right)+\varepsilon^{2} w_{2,0,0}\left(\zeta ; x_{1}\right)+\cdots,
$$

where

$$
w_{2,0,0} \sim \alpha_{2}^{0}, \quad \tau \rightarrow+\infty .
$$

We now give the boundary-value problem for $w_{2,0,0}$. We substitute the expansion (60) into problem (2), rewritten in terms of the variables $\zeta$, and collect terms with the powers 
$a^{-2}$ in the equation and with the powers $\varepsilon a^{-1}$ in the boundary condition. In view of (61) we obtain the problem

$$
\left\{\begin{aligned}
\Delta_{\zeta} w_{2,0,0} & =0 \quad \text { in } \mathbb{R}_{+}^{2}, \\
\frac{\partial w_{2,0,0}}{\partial \zeta_{2}}=0 \quad \text { on } \Gamma & \\
w_{2,0,0} & =0 \quad \text { on } \gamma \\
w_{2,0,0} & \sim \alpha_{2}^{0} \quad \text { as } \tau \rightarrow+\infty
\end{aligned}\right.
$$

Obviously, (62) is solvable only for

$$
\alpha_{2}^{0}\left(x_{1}\right) \equiv 0,
$$

and the function

$$
w_{2,0,0}\left(\zeta ; x_{1}\right) \equiv 0
$$

is a solution. Therefore we define $\alpha_{2}^{0}$ and $w_{2,0,0}$ in accordance with (63) and (64). Then it follows from (54) that $v_{2}$ has the final form

$$
v_{2}\left(\xi ; x_{1}\right)=\left(\alpha_{0}^{1}\right)^{\prime}\left(x_{1}\right) X_{2}(\xi) .
$$

In view of (49) and (63), the boundary-value problem (13) for $j=2$ takes the form

$$
\left\{\begin{aligned}
-\Delta u_{2} & =\lambda_{0} u_{2}+\lambda_{2} u_{0} \quad \text { for } x \in \Omega, \\
u_{2} & =0 \quad \text { for } x \in \Gamma_{4}, \\
\frac{\partial u_{2}}{\partial \nu} & =0 \quad \text { for } x \in \Gamma_{1} \cup \Gamma_{2} \cup \Gamma_{3} .
\end{aligned}\right.
$$

It is also obvious that the pair

$$
u_{2}(x)=0, \quad \lambda_{2}=0
$$

satisfies (66). Hence, $\alpha_{2}^{l} \equiv 0$ for $d l=1,2, \ldots$.

We now rewrite problem (24) for $j=3$ taking (57) into account:

$$
\left\{\begin{aligned}
-\Delta_{\xi} v_{3} & =2 \frac{\partial^{2} v_{2}}{\partial x_{1} \partial \xi_{1}}+\frac{\partial^{2} v_{1}}{\partial x_{1}^{2}}+\lambda_{0} v_{1} \quad \text { in } \Pi, \\
\frac{\partial v_{3}}{\partial \xi_{2}} & =0 \quad \text { on } \Sigma \\
\frac{\partial v_{3}}{\partial \xi_{1}}\left(\xi ; \pm \frac{\pi}{2}\right) & =0 \quad \text { at } \xi_{1}= \pm \frac{\pi}{2} \\
v_{3} & \sim V_{3}\left(\xi_{2} ; x_{1}\right) \quad \text { as } \xi_{2} \rightarrow+\infty .
\end{aligned}\right.
$$

We observe that by (54) and (63), as well as by (31) and (46), the right-hand side of the equation in problem (68) takes the following form:

$$
F=2\left(\alpha_{0}^{1}\right)^{\prime \prime} \frac{\partial X_{2}}{\partial \xi_{1}}+\left(\alpha_{0}^{1}\right)^{\prime \prime} X+\lambda_{0} \alpha_{0}^{1} X
$$

For our convenience when we analyse problems with a right-hand side of the form (69), we introduce the following classes of functions.

- We let $A_{\text {even }}^{(2 k, q)}$ denote the set of functions

$$
f_{\text {even }} \in C^{\infty}\left(\overline{\mathbb{R}_{+}^{2}} \backslash \bigcup_{k \in \mathbb{Z}}\{(k \pi, 0)\}\right)
$$


that are $\pi$-periodic and even in $\xi_{1}$, have asymptotic behaviour at zero of the form

$$
\begin{aligned}
f_{\text {even }}(\xi)= & \delta_{0}^{q} \sum_{n=1}^{k+1} \beta_{\text {even }}^{(n)} \rho^{2 n-2} \cos 2 n \theta+\sum_{n=0}^{k} \sum_{j=0}^{n} \widehat{\beta}_{\text {even }}^{(n, j)} \rho^{2 n} \cos 2(n-j) \theta \ln \rho \\
& +\sum_{n=0}^{\infty} \sum_{j=0}^{n} \widetilde{\beta}_{\text {even }}^{(n, j)} \rho^{2 n} \cos 2(n-j) \theta, \quad \rho \rightarrow 0,
\end{aligned}
$$

where $\delta_{0}^{q}$ is the Kronecker delta, and have the property

$$
e^{\varpi \xi_{2}} \chi(\rho) f_{\text {even }} \in H^{1}(\Pi), \quad 0<\varpi<2 .
$$

It follows from the definition of $X(\xi)$ (see (28)) and $X_{2}(\xi)$ (see Lemma 3.1) that

$$
\frac{\partial X_{2}}{\partial \xi_{1}} \in A_{\text {even }}^{(0,0)}, \quad X=\xi_{2}+\tilde{X}, \quad \tilde{X} \in A_{\text {even }}^{(0,1)} .
$$

We also note that

$$
A_{\text {even }}^{(2 k, 1)} \subset A_{\text {even }}^{(2 k+2 s, 1)}, \quad A_{\text {even }}^{(2 k, 0)} \subset A_{\text {even }}^{(2 k+2 s, 0)}, \quad A_{\text {even }}^{(2 k, 1)} \subset A_{\text {even }}^{(2 k+2 s, 0)}, \quad s=0,1, \ldots
$$

We set

$$
\begin{aligned}
\mathcal{A}_{\text {even }}^{(2 k, q)}=\left\{V\left(\xi ; x_{1}\right):\right. & V\left(\xi ; x_{1}\right)=\sum_{j=1}^{J} \sigma_{j}\left(x_{1}\right) Y_{j}(\xi), \quad Y_{j} \in A_{\text {even }}^{(2 k, q)} \\
& \left.\sigma_{j} \in C^{\infty}\left[-\frac{\pi}{2}, \frac{\pi}{2}\right], \quad\left(\sigma_{j}\right)^{(2 n+1)}\left( \pm \frac{\pi}{2}\right)=0, n=0,1, \ldots\right\},
\end{aligned}
$$

where $J$ is arbitrary (not fixed but finite). Note that

$$
\frac{\partial V}{\partial x_{1}}\left(\xi ; \pm \frac{\pi}{2}\right)=0 \quad \text { for all } \quad V \in \mathcal{A}_{\text {even }}^{(2 k, q)} .
$$

Thus, it follows from (14) and (171) that the right-hand side of the equation in problem (68) (see (69)) can be represented in the form

$$
F=\mathcal{P}_{1}\left(\xi_{2} ; x_{1}\right)+f
$$

where

$$
\mathcal{P}_{1}\left(\xi_{2} ; x_{1}\right)=\left(\left(\alpha_{0}^{1}\right)^{\prime \prime}+\lambda_{0} \alpha_{0}^{1}\right) \xi_{2}, \quad-\Delta_{\xi} V_{3}=\mathcal{P}_{1}, \quad f \in \mathcal{A}_{\text {even }}^{(0,0)} .
$$

Here, and in what follows, $\mathcal{P}_{t}\left(\xi_{2} ; x_{1}\right)$ denotes a polynomial of order $t$ in the variable $\xi_{2}$ whose coefficients are functions of $x_{1}$ that are infinitely differentiable on $[-\pi / 2, \pi / 2]$ and have odd derivatives vanishing at $x_{1}= \pm \pi / 2$.

Lemma 3.2. a) Let $F$ be represented in the form $F=\mathcal{P}_{t}+f$, where $f \in \mathcal{A}_{\text {even }}^{(2 k, 0)}$ and $\mathcal{P}_{t+2}$ is a polynomial such that $-\mathcal{P}_{t+2}^{\prime \prime}=\mathcal{P}_{t}$. Then there exists a solution of the boundary-value problem

$$
\left\{\begin{aligned}
-\Delta_{\xi} W & =F \quad \text { in } \quad \Pi, \\
\frac{\partial W}{\partial \xi_{2}} & =0 \quad \text { on } \quad \Sigma, \\
\frac{\partial W}{\partial \xi_{1}} & =0 \quad \text { at } \quad \xi_{1}= \pm \frac{\pi}{2}
\end{aligned}\right.
$$

that can be represented in the form $W=\mathcal{P}_{t+2}+W_{1}$, where $W_{1} \in \mathcal{A}_{\mathrm{even}}^{(2 k+2,1)}$.

b) Furthermore,

at $x_{1}= \pm \pi / 2$.

$$
\frac{\partial W}{\partial x_{1}}\left(\xi ; \pm \frac{\pi}{2}\right)=0, \quad \frac{\partial W}{\partial x_{1}}\left(\frac{x}{\varepsilon} ; x_{1}\right)=0
$$


This lemma is proved in Appendix 1 (\$4).

By (17) the polynomial $V_{3}\left(\xi_{2} ; x_{1}\right)$ can be represented in the form

$$
V_{3}=\widetilde{V}_{3}+\alpha_{3}^{0},
$$

where $\widetilde{V}_{3}=\mathcal{P}_{3}\left(\xi_{2} ; x_{1}\right)$ is a given polynomial, independent of $\alpha_{3}^{0}$ (it was defined before this step). By Lemma 3.2 and (73) there exists a solution of the boundary-value problem (68) which can be represented in the form

$$
v_{3}=V_{3}+\widetilde{v}_{3},
$$

where $\widetilde{v}_{3} \in \mathcal{A}_{\text {even }}^{(2,1)}$ is a completely defined function that is independent of $\alpha_{3}^{0}$. Note that

$$
\frac{\partial v_{3}}{\partial x_{1}}\left(\xi ; x_{1}\right)=0 \quad \text { at } x_{1}= \pm \frac{\pi}{2}, \quad \frac{\partial v_{3}}{\partial \nu}\left(\frac{x}{\varepsilon} ; x_{1}\right)=0 \quad \text { on } \Gamma_{2} \cup \Gamma_{3} .
$$

It follows from (76) that $v_{3}$ has asymptotics at zero of the form

$$
v_{3}\left(\xi ; x_{1}\right)=\mathcal{M}_{3}\left(x_{1}\right) \ln \rho+\widehat{\mathcal{M}}_{3}\left(x_{1}\right)+\alpha_{3}^{0}\left(x_{1}\right)+O\left(\rho^{2} \ln \rho\right) \quad \text { as } \quad \rho \rightarrow 0,
$$

where $\mathcal{M}_{3}\left(x_{1}\right)$ and $\widehat{\mathcal{M}}_{3}\left(x_{1}\right)$ are completely defined functions such that

$$
\frac{d^{2 j+1} \mathcal{M}_{3}}{d x_{1}^{2 j+1}}\left( \pm \frac{\pi}{2}\right)=0, \quad \frac{d^{2 j+1} \widehat{\mathcal{M}}_{3}}{d x_{1}^{2 j+1}}\left( \pm \frac{\pi}{2}\right)=0, \quad j=0, \ldots .
$$

Recall also that $\alpha_{3}^{0}\left(x_{1}\right)$ is some, so far undefined, function (completely analogous to $\alpha_{1}^{0}\left(x_{1}\right)$ in the definition (31) of the function $v_{1}$ ).

We rewrite (77), which gives the asymptotics of the function $v_{3}$ at zero, in terms of $\zeta$. As $a \tau \rightarrow 0$, we have

$$
\begin{aligned}
\varepsilon^{3} v_{3}\left(a \zeta ; x_{1}\right)= & \varepsilon^{3} \mathcal{M}_{3}\left(x_{1}\right)[\ln a+\ln \tau]+\varepsilon^{3} \widehat{\mathcal{M}}_{3}\left(x_{1}\right) \\
& +\varepsilon^{3} \alpha_{3}^{0}\left(x_{1}\right)+O\left(\varepsilon^{3}(a \tau)^{2} \ln (a \tau)\right) .
\end{aligned}
$$

Rewriting the asymptotics of the series (18) as $\rho \rightarrow 0$ in terms of $\zeta$, taking (79) and (34) into account and following the method of matched asymptotic expansions, we conclude that the expansion of the function $u_{\varepsilon}$ in the new variables $\zeta$ must have the form

$$
\begin{aligned}
u_{\varepsilon}= & \varepsilon \ln a w_{1,1,0}\left(\zeta ; x_{1}\right)+\varepsilon w_{1,0,0}\left(\zeta ; x_{1}\right)+\varepsilon^{2} a \ln a w_{2,1,1}\left(\zeta ; x_{1}\right) \\
& +\varepsilon^{2} a w_{2,0,1}+\varepsilon^{3} \ln a w_{3,1,0}\left(\zeta ; x_{1}\right)+\varepsilon^{3} w_{3,0,0}\left(\zeta ; x_{1}\right)+\cdots,
\end{aligned}
$$

where, as $\tau \rightarrow+\infty$, the term $w_{3,0,0}$ has asymptotic behaviour

$$
w_{3,0,0} \sim \mathcal{M}_{3} \ln \tau+\widehat{\mathcal{M}}_{3}+\alpha_{3}^{0} .
$$

We rewrite the operator in problem (2) in terms of the inner variables $\zeta$ (see (40)), substitute the expansion (80) and the series (12) into (2) and, in the equation, collect terms in powers of $\varepsilon a^{-2}$, and in the boundary conditions, in powers of $\varepsilon^{2} a^{-1}$, respectively. In view of (81) we obtain a problem for the function $w_{3,0,0}$ of the form

$$
\left\{\begin{aligned}
\Delta_{\zeta} w_{3,0,0} & =0 \quad \text { in } \mathbb{R}_{+}^{2}, \\
\frac{\partial w_{3,0,0}}{\partial \zeta_{2}} & =0 \quad \text { on } \Gamma \\
w_{3,0,0} & =0 \quad \text { on } \gamma, \\
w_{3,0,0} & \sim \mathcal{M}_{3} \ln \tau+\widehat{\mathcal{M}}_{3}+\alpha_{3}^{0} \quad \text { as } \tau \rightarrow+\infty .
\end{aligned}\right.
$$

Taking into account the asymptotic behaviour in (44) and problem (43), we conclude that the function

$$
w_{3,0,0}\left(\zeta ; x_{1}\right)=\mathcal{M}_{3}\left(x_{1}\right) Y(\zeta)
$$


is a solution of problem (82) for

$$
\alpha_{3}^{0}\left(x_{1}\right)=\mathcal{M}_{3} \ln 2-\widehat{\mathcal{M}}_{3},
$$

where $Y(\zeta)$ is the function defined in (42). Therefore we fix $w_{3,0,0}$ and $\alpha_{3}^{0}$ in accordance with (83) and (84). We observe that by (78) condition (14) holds. We also point out that this completes the construction of the function $v_{3}$.

Taking account of (49) and (67), we obtain the boundary-value problem (13) for $j=3$ :

$$
\left\{\begin{aligned}
-\Delta u_{3} & =\lambda_{0} u_{3}+\lambda_{3} u_{0} \quad \text { for } x \in \Omega, \\
u_{3} & =\alpha_{3}^{0} \quad \text { for } x \in \Gamma_{4}, \\
\frac{\partial u_{3}}{\partial \nu} & =0 \quad \text { for } x \in \Gamma_{1} \cup \Gamma_{2} \cup \Gamma_{3},
\end{aligned}\right.
$$

where $\alpha_{3}^{0}$ is the function we have just defined in (84). From the condition that this problem be solvable, we determine $\lambda_{3}$ and, correspondingly, find $u_{3}$.

Thus, because we have completely determined the function $v_{3}\left(\xi ; x_{1}\right)$ compatible with $w_{3,0,0}$, we have found the boundary condition for $u_{3}$. In other words, we have constructed $v_{3}, w_{3,0,0}, u_{3}$, and $\lambda_{3}$ in this step.

In what follows, we will construct $\lambda_{j}, u_{j}, v_{j}$, and $w_{j, 0,0}$ in a similar way.

We will be looking at (24) with special right-hand sides, and for convenience we introduce the following classes of functions.

- We denote by $A_{\text {odd }}^{(2 k-1, q)}$ the set of functions $f_{\text {odd }} \in C^{\infty}\left(\overline{\mathbb{R}_{+}^{2}} \backslash \bigcup_{k \in \mathbb{Z}}\{(k \pi, 0)\}\right)$ that are $\pi$-periodic and odd in $\xi_{1}$, have asymptotic behaviour at zero of the form

$$
\begin{aligned}
f_{\text {odd }}(\xi)= & \delta_{0}^{q} \sum_{n=0}^{k} \beta_{\text {odd }}^{(n)} \rho^{2 n-1} \cos (2 n+1) \theta \\
& +\sum_{n=0}^{k-1} \sum_{j=0}^{n} \widehat{\beta}_{\text {odd }}^{(n, j)} \rho^{2 n+1} \cos (2 n-2 j+1) \theta \ln \rho \\
& +\sum_{n=0}^{\infty} \sum_{j=0}^{n} \widetilde{\beta}_{\text {odd }}^{(n, j)} \rho^{2 n+1} \cos (2 n-2 j+1) \theta, \quad \rho \rightarrow 0,
\end{aligned}
$$

where $\delta_{0}^{q}$ is the Kronecker delta, and have the property

$$
e^{\varpi \xi_{2}} \chi(\rho) f_{\text {odd }} \in H^{1}(\Pi), \quad 0<\varpi<2 .
$$

We note here that the symbol $A_{\text {odd }}^{(-1, q)}$ (that is, when $k=0$ ) denotes the class of functions whose asymptotics as $\rho \rightarrow 0$ do not have summands involving $\ln \rho$.

We observe that

$$
\begin{gathered}
A_{\text {odd }}^{(2 k-1,1)} \subset A_{\text {odd }}^{(2 k+2 s-1,1)}, \quad A_{\text {odd }}^{(2 k-1,0)} \subset A_{\text {odd }}^{(2 k+2 s-1,0)}, \\
A_{\text {odd }}^{(2 k-1,1)} \subset A_{\text {odd }}^{(2 k+2 s-1,0)}, \quad s=0,1, \ldots, \\
\frac{\partial X}{\partial \xi_{1}} \in A_{\text {odd }}^{(-1,0)}, \quad X_{2} \in A_{\text {odd }}^{(1,1)} .
\end{gathered}
$$

We also set

$$
\begin{aligned}
\mathcal{A}_{\text {odd }}^{(2 k-1, q)}=\left\{V\left(\xi ; x_{1}\right):\right. & V\left(\xi ; x_{1}\right)=\sum_{j=1}^{J} \sigma_{j}\left(x_{1}\right) Y_{j}(\xi), Y_{j} \in A_{\text {odd }}^{(2 k-1, q)}, \\
& \left.\sigma_{j} \in C^{\infty}\left[-\frac{\pi}{2}, \frac{\pi}{2}\right],\left(\sigma_{j}\right)^{(2 n)}\left( \pm \frac{\pi}{2}\right)=0, n=0,1, \ldots\right\},
\end{aligned}
$$

where $J$ is arbitrary (not fixed and finite). 
It follows immediately from the definition of these classes, as well as from (14), that the following hold.

1. If $V \in \mathcal{A}_{\text {odd }}^{(2 k-1,1)}$, then $\frac{\partial^{2} V}{\partial \xi_{1} \partial x_{1}} \in \mathcal{A}_{\text {even }}^{(2 k-2,0)}$ and $\frac{\partial^{2} V}{\partial x_{1}^{2}} \in \mathcal{A}_{\text {odd }}^{(2 k-1,1)}$.

2. If $V \in \mathcal{A}_{\text {even }}^{(2 k, 1)}, \quad$ then $\frac{\partial^{2} V}{\partial \xi_{1} \partial x_{1}} \in \mathcal{A}_{\text {odd }}^{(2 k-1,0)}$ and $\frac{\partial^{2} V}{\partial x_{1}^{2}} \in \mathcal{A}_{\text {even }}^{(2 k, 1)}$.

We introduce the following classes of functions:

$$
\begin{aligned}
\mathbb{A}^{(2 k+1, q)} & =\left\{V\left(\xi ; x_{1}\right): \quad V\left(\xi ; x_{1}\right)=\widehat{Z}\left(\xi ; x_{1}\right)+\check{Z}\left(\xi ; x_{1}\right), \widehat{Z} \in \mathcal{A}_{\text {odd }}^{(2 k+1, q)}, \quad \check{Z} \in \mathcal{A}_{\text {even }}^{(2 k, q)}\right\}, \\
\mathbb{A}^{(2 k, q)} & =\left\{V\left(\xi ; x_{1}\right): \quad V\left(\xi ; x_{1}\right)=\widehat{Z}\left(\xi ; x_{1}\right)+\check{Z}\left(\xi ; x_{1}\right), \widehat{Z} \in \mathcal{A}_{\text {odd }}^{(2 k-1, q)}, \quad \check{Z} \in \mathcal{A}_{\text {even }}^{(2 k, q)}\right\},
\end{aligned}
$$

where $J$ is arbitrary (finite but not fixed).

These classes satisfy:

$$
\mathbb{A}^{(k, 1)} \subset \mathbb{A}^{(k+2 s, 1)}, \quad \mathbb{A}^{(k, 0)} \subset \mathbb{A}^{(k+2 s, 0)}, \quad \mathbb{A}^{(k, 1)} \subset \mathbb{A}^{(k+2 s, 0)}, \quad s=0,1, \ldots
$$

Theorem 3.1. a) Let $F$ be represented in the form $F=\mathcal{P}_{t}+f$, where $f \in \mathbb{A}^{(k, 0)}$ and $\mathcal{P}_{t+2}$ is a polynomial such that $-\mathcal{P}_{t+2}^{\prime \prime}=\mathcal{P}_{t}$. Then there exists a solution of the boundary-value problem

$$
\left\{\begin{aligned}
-\Delta_{\xi} W & =F \quad \text { in } \Pi, \\
\frac{\partial W}{\partial \xi_{2}} & =0 \quad \text { on } \Sigma, \\
\frac{\partial W}{\partial \xi_{1}} & =0 \quad \text { at } \xi_{1}= \pm \frac{\pi}{2}, x_{1}= \pm \frac{\pi}{2},
\end{aligned}\right.
$$

that can be represented in the form $W=\mathcal{P}_{t+2}+W_{1}$, where $W_{1} \in \mathbb{A}^{(k+2,1)}$.

b) The function $W$ satisfies the equalities

$$
\begin{aligned}
& \frac{\partial W}{\partial x_{1}}\left(\xi ; \pm \frac{\pi}{2}\right)=0 \quad \text { at } \quad \xi_{1}= \pm \frac{\pi}{2}, \\
& \frac{\partial W}{\partial x_{1}}\left(\frac{x}{\varepsilon} ; x_{1}\right)=0 \quad \text { at } \quad x_{1}= \pm \frac{\pi}{2} .
\end{aligned}
$$

The proof of the theorem will be given below in Appendix 1 (\$4).

Lemma 3.3. a) Suppose that

$$
V\left(\xi ; x_{1}\right)=\sum_{k=1}^{\infty} \varepsilon^{k} v_{k}\left(\xi ; x_{1}\right),
$$

where $v_{k}=\mathcal{P}_{k}+\widetilde{v}_{k}$ for $\widetilde{v}_{k} \in \mathbb{A}^{(k, 1)}$ with asymptotics of the form $\widetilde{v}_{k}=\ln \rho \mathcal{M}_{k}+\check{\mathcal{M}}_{k}$ as $\rho \rightarrow 0$. Then the asymptotics of the series $V$ as $\rho \rightarrow 0$ rewritten in terms of $\zeta$ can be represented in the form

$$
\begin{aligned}
V\left(\xi ; x_{1}\right)= & \ln a \sum_{k=1}^{\infty} \varepsilon^{k} \mathcal{M}_{k}\left(x_{1}\right)+\sum_{k=1}^{\infty} \varepsilon^{k}\left(\mathcal{M}_{k}\left(x_{1}\right) \ln \tau+\check{\mathcal{M}}_{k}\left(x_{1}\right)\right) \\
& +O(a \tau \ln (a \tau)) \quad \text { as } \quad \text { a } \tau \rightarrow 0 .
\end{aligned}
$$

Furthermore,

$$
\frac{d^{2 j+1} \mathcal{M}_{k}}{d x_{1}^{2 j+1}}\left( \pm \frac{\pi}{2}\right)=0, \quad \frac{d^{2 j+1} \check{\mathcal{M}}_{k}}{d x_{1}^{2 j+1}}\left( \pm \frac{\pi}{2}\right)=0, \quad j=0, \ldots
$$


b) If in addition the $v_{j}$ are solutions of the problems

$$
\left\{\begin{array}{l}
-\Delta_{\xi} v_{j}=2 \frac{\partial^{2} v_{j-1}}{\partial x_{1} \partial \xi_{1}}+\frac{\partial^{2} v_{j-2}}{\partial x_{1}^{2}}+\sum_{k=0}^{j-2} \lambda_{k} v_{j-2-k} \quad \text { in } \Pi, \\
\frac{\partial v_{j}}{\partial \xi_{2}}=0 \quad \text { on } \Sigma \\
\frac{\partial v_{j}}{\partial \xi_{1}}\left(\xi ; \pm \frac{\pi}{2}\right)=0 \quad \text { at } \xi_{1}= \pm \frac{\pi}{2} \\
v_{j}=V_{j}+o(1) \quad \text { as } \xi_{2} \rightarrow+\infty
\end{array}\right.
$$

then the $v_{j}$ are solutions of the problems (24).

Proof. The assertion of part a) follows immediately from the definition of the class $\mathbb{A}^{(k, 1)}$. We will prove part $\mathrm{b}$ ). Let the $v_{j}$ be solutions of the homogeneous boundary-value problems (92). It follows from part b) of Theorem 3.1 that

$$
\frac{\partial v_{j}}{\partial x_{1}}\left(\xi ; \pm \frac{\pi}{2}\right)=0
$$

for all $j=1,2, \ldots$ and, consequently, the $v_{j}$ are solutions of the problems (24).

We seek the terms of the intermediate expansion $v_{k}$ that can be represented in the form of the sum of the polynomial $V_{k}$ and a function in the class $\mathbb{A}^{(k, 1)}$ and are solutions of (92) and, consequently, by part b) of Lemma 3.3. also solutions of (24). Notice that the functions $v_{1}, v_{2}$, and $v_{3}$ constructed earlier possess these properties.

Following the method of matched asymptotic expansions, by part a) of Lemma 3.3 we deduce from the representation (90) that the inner expansion must be sought in the form

$$
u_{\varepsilon}=\sum_{k=1}^{\infty} \varepsilon^{k} \ln a w_{k, 1,0}+\sum_{k=1}^{\infty} \varepsilon^{k} w_{k, 0,0}+\cdots,
$$

where the term $w_{k, 0,0}$ has an asymptotic as $\tau \rightarrow+\infty$ of the form

$$
w_{k, 0,0} \sim \mathcal{M}_{k} \ln \tau+\check{\mathcal{M}}_{k},
$$

while the term $w_{1,1,0}$ has an asymptotic as $\tau \rightarrow+\infty$ of the form

$$
w_{1,1,0} \sim \mathcal{M}_{1} .
$$

We rewrite the operator of the problem (2) in terms of the inner variables $\zeta$, substitute the expansion (93) and the series (12) into the problem (2), and collect terms with like powers of $\varepsilon, a$, and $\ln a$ (in the equation with $\varepsilon^{k-2} a^{-2}$, in the boundary conditions with $\left.\varepsilon^{k-1} a^{-1}\right)$. Taking the asymptotic behaviour in (94) into account, the functions $w_{k, 0,0}$ must satisfy problems of the form

$$
\left\{\begin{aligned}
\Delta_{\zeta} w_{k, 0,0} & =0 \quad \text { in } \mathbb{R}_{+}^{2}, \\
\frac{\partial w_{k, 0,0}}{\partial \zeta_{2}} & =0 \quad \text { on } \Gamma, \\
w_{k, 0,0} & =0 \quad \text { on } \gamma, \\
w_{k, 0,0} & \sim \mathcal{M}_{k} \ln \tau+\check{\mathcal{M}}_{k} \quad \text { as } \tau \rightarrow+\infty .
\end{aligned}\right.
$$

Obviously, if

$$
\check{\mathcal{M}}_{k}=\mathcal{M}_{k} \ln 2
$$


then the problem turns into

$$
\left\{\begin{aligned}
\Delta_{\zeta} w_{k, 0,0} & =0 \quad \text { in } \mathbb{R}_{+}^{2} \\
\frac{\partial w_{k, 0,0}}{\partial \zeta_{2}} & =0 \quad \text { on } \Gamma \\
w_{k, 0,0} & =0 \quad \text { on } \gamma \\
w_{k, 0,0} & \sim \mathcal{M}_{k}(\ln \tau+\ln 2) \quad \text { as } \tau \rightarrow+\infty
\end{aligned}\right.
$$

and the functions

$$
w_{k, 0,0}=\mathcal{M}_{k} Y
$$

are solutions of (98).

We now show that we can satisfy (97) by finding all the $\alpha_{k}^{0}$ in turn. Moreover, we have already done this for $k=1,2,3$ by choosing $\alpha_{1}^{0}, \alpha_{2}^{0}$, and $\alpha_{3}^{0}$ in accordance with (46), (63), and (84).

We will construct the rest by induction.

Suppose that $\alpha_{k}^{0}, v_{k}, u_{k}$, and $\lambda_{k}(k \leq n)$ are defined such that

$(\phi 1)$ the $\alpha_{k}^{0}$ satisfy (14);

$(\phi 2)$ the pairs $u_{k} \in C^{\infty}(\bar{\Omega})$ and $\lambda_{k}$ satisfy (13);

( $\phi 3) v_{k}=V_{k}+\widetilde{v}_{k}, \widetilde{v}_{k} \in \mathbb{A}^{(k, 1)}$, and the $v_{k}$ are solutions of (92) (and also of (24) by part b) of Lemma 3.3);

$(\phi 4)$ the $v_{k}$ have the asymptotic behaviour

$$
v_{k}\left(\xi ; x_{1}\right)=\mathcal{M}_{k}\left(x_{1}\right)(\ln \rho+\ln 2)+O(\rho \ln \rho) \quad \text { as } \quad \rho \rightarrow 0,
$$

where the $\mathcal{M}_{k}$ satisfy (91).

Remark 3.4. We emphasize that we have already determined $\alpha_{1}^{0}, \alpha_{2}^{0}$, and $\alpha_{3}^{0}, u_{1}, u_{2}$, and $u_{3}, \lambda_{1}, \lambda_{2}^{0}$, and $\lambda_{3}^{0}$, as well as $v_{1}, v_{2}$, and $v_{3}$ such that assertions $(\phi 1)-(\phi 4)$ hold. Note, in particular, that

$$
\mathcal{M}_{1}=\alpha_{0}^{1}
$$

Consider the $(n+1)$ th step. By (17), the polynomial $V_{n+1}$ can be represented in the form

$$
V_{n+1}=\widetilde{V}_{n+1}+\alpha_{n+1}^{0}
$$

where $\widetilde{V}_{n+1}=\mathcal{P}_{n+1}$ is a given polynomial (defined earlier) that is independent of $\alpha_{n+1}^{0}$. By part a) of Theorem 3.1 and (25) there exists a solution of the boundary-value problem (92) (for $j=n+1$ ) that can be represented in the form

$$
v_{n+1}=V_{n+1}+\widetilde{v}_{n+1},
$$

where $\widetilde{v}_{n+1} \in \mathbb{A}^{(n+1,1)}$ is a completely defined function that is independent of $\alpha_{n+1}^{0}$. Thus, part $(\phi 3)$ holds for $k=n+1$.

By (103), the definition of the class $\mathbb{A}^{(n, 1)}$ implies that at zero the function $v_{n+1}$ has the asymptotic behaviour

$$
v_{n+1}\left(\xi ; x_{1}\right)=\mathcal{M}_{n+1}\left(x_{1}\right) \ln \rho+\widehat{\mathcal{M}}_{n+1}\left(x_{1}\right)+\alpha_{n+1}^{0}\left(x_{1}\right)+O(\rho \ln \rho) \quad \text { as } \quad \rho \rightarrow 0,
$$

where

$$
\frac{d^{2 j+1} \mathcal{M}_{n+1}}{d x_{1}^{2 j+1}}\left( \pm \frac{\pi}{2}\right)=0, \quad \frac{d^{2 j+1} \widehat{\mathcal{M}}_{n+1}}{d x_{1}^{2 j+1}}\left( \pm \frac{\pi}{2}\right)=0, \quad j=0,1, \ldots
$$

We set

$$
\alpha_{n+1}^{0}\left(x_{1}\right)=\mathcal{M}_{n+1} \ln 2-\widehat{\mathcal{M}}_{n+1} .
$$


Then in view of (105), parts $(\phi 1)$ and $(\phi 4)$ hold for $k=n+1$.

Taking (49) and (67) into account, we rewrite the boundary-value problem (13) for $j=n+1$ :

$$
\left\{\begin{aligned}
-\Delta u_{n+1} & =\lambda_{0} u_{n+1}+\lambda_{3} u_{n-2}+\cdots+\lambda_{n+1} u_{0} \quad \text { for } x \in \Omega, \\
u_{n+1} & =\alpha_{n+1}^{0} \quad \text { for } x \in \Gamma_{4}, \\
\frac{\partial u_{n+1}}{\partial \nu} & =0 \quad \text { for } x \in \Gamma_{1} \cup \Gamma_{2} \cup \Gamma_{3},
\end{aligned}\right.
$$

where $\alpha_{n+1}^{0}$ is the function already defined by equality (106). As this problem is solvable, we determine $\lambda_{n+1}$ and, correspondingly, find $u_{n+1} \in C^{\infty}(\bar{\Omega})$. Thus, part $(\phi 2)$ holds for $k=n+1$. Recall that $w_{n+1}$ is chosen in accordance with (99).

As a result we have proved the following theorem.

Theorem 3.2. There exist series (11), (12), (18), and (93) such that

a) the pairs $u_{j} \in C^{\infty}(\bar{\Omega})$ and $\lambda_{j}$ are solutions of the problems (13);

b) the coefficients satisfy $\lambda_{1}=\lambda_{2}=0$ and $u_{1} \equiv 0, u_{2} \equiv 0$;

c) the functions $v_{j}$ can be represented as the sums $v_{j}=V_{j}+\widetilde{v}_{j}, \widetilde{v}_{j} \in \mathbb{A}^{(j, 1)}$, and are solutions of the problems (24);

d) the series (18) has asymptotic behaviour as $\rho \rightarrow 0$, rewritten in the variables $\zeta$, that has the form

$$
\begin{aligned}
u_{\varepsilon}= & \ln a \sum_{k=1}^{\infty} \varepsilon^{k} \mathcal{M}_{k}\left(x_{1}\right)+\sum_{k=1}^{\infty} \varepsilon^{k} \mathcal{M}_{k}\left(x_{1}\right)(\ln \tau+\ln 2) \\
& +O(a \tau \ln (a \tau)) \quad \text { as } \quad a \tau \rightarrow 0,
\end{aligned}
$$

where

$$
\frac{d^{2 j+1} \mathcal{M}_{k}}{d x_{1}^{2 j+1}}\left( \pm \frac{\pi}{2}\right)=0, \quad j=0,1, \ldots ;
$$

e) the coefficients of the series (93), $w_{k, 0,0}=\mathcal{M}_{k} Y$, are solutions of problems (98).

Remark 3.5. In the asymptotic expansion (108) the last expression $O(a \tau \ln (a \tau))$ denotes an infinite sum of terms each of which has this order.

This finishes the construction of the terms in powers of $\varepsilon$.

3.2. The construction of the asymptotics. Second step. In this subsection we assume that the coefficients $v_{k}, u_{k}, \lambda_{k}$, and $w_{k, 0,0}$ have all been constructed according to the scheme in the preceding subsection; that is, they all satisfy the statement of Theorem 3.2.

We now return to the terms $w_{k, 1,0}$ in the expansion (93). We give the problem for the function $w_{1,1,0}$. We rewrite the operator of problem (2) in the inner variables $\zeta$, substitute the expansion (93) and the series (12) into (21), and collect terms with like powers of $\varepsilon, a$, and $\ln a$ (in the equation with $\varepsilon^{-1} a^{-2} \ln a$, in the boundary conditions with $a^{-1} \ln a$ ). In view of (95) and (101) the problem has the form

$$
\left\{\begin{aligned}
\Delta_{\zeta} w_{1,1,0} & =0 \quad \text { in } \mathbb{R}_{+}^{2}, \\
\frac{\partial w_{1,1,0}}{\partial \zeta_{2}}=0 \quad \text { on } \Gamma & \\
w_{1,1,0} & =0 \quad \text { on } \gamma \\
w_{1,1,0} & \sim \alpha_{0}^{1} \quad \text { as } \tau \rightarrow+\infty
\end{aligned}\right.
$$


This problem has no solution; therefore the term $w_{1,1,0}$ cannot be present in the expansion. So that this can happen, in the intermediate expansion we introduce the additional term $\varepsilon \ln a v_{1,1}$ with asymptotic behaviour

$$
v_{1,1} \sim-\alpha_{0}^{1} \quad \text { as } \quad \rho \rightarrow 0
$$

that is, the expansion takes the form

$$
u_{\varepsilon}(x)=\sum_{j=1}^{+\infty} \varepsilon^{j} v_{j}\left(\frac{x}{\varepsilon} ; x_{1}\right)+\varepsilon \ln a v_{1,1}\left(\frac{x}{\varepsilon} ; x_{1}\right)+\cdots .
$$

Rewriting the asymptotics of the series (112) as $\rho \rightarrow 0$ in terms of the inner variables $\zeta$, we see that, by (111) and (108), the asymptotics takes the form

$$
\begin{aligned}
u_{\varepsilon}= & \ln a \sum_{k=2}^{\infty} \varepsilon^{k} \mathcal{M}_{k}\left(x_{1}\right)+\sum_{k=1}^{\infty} \varepsilon^{k} \mathcal{M}_{k}\left(x_{1}\right)(\ln \tau+\ln 2) \\
& +O(a \tau \ln (a \tau)) \quad \text { as } \quad a \tau \rightarrow 0,
\end{aligned}
$$

where the $\mathcal{M}_{k}$ are the same as before. Next, by following the matc hing method, we conclude that the inner expansion has the form

$$
u_{\varepsilon}=\sum_{k=2}^{\infty} \varepsilon^{k} \ln a w_{k, 1,0}+\sum_{k=1}^{\infty} \varepsilon^{k} w_{k, 0,0}+\cdots ;
$$

that is, in the expansion (93) there is no term $w_{1,1,0}$, while the asymptotics as $\tau \rightarrow+\infty$ for the terms $w_{k, 0,0}$ have not changed and therefore they remain to be chosen in accordance with (99).

Substituting the series (112) and the expansion (12) into the problem (2) (first having rewritten it in terms of the coordinates $\xi$; see (20) ) and collecting terms in powers $\varepsilon^{-1} \ln a$ in the equation and in $\ln a$ in the boundary conditions, by using (111) we conclude that the problem for $v_{1,1}$ has the form

$$
\left\{\begin{array}{l}
\Delta_{\xi} v_{1,1}=0 \quad \text { in } \Pi \\
\frac{\partial v_{1,1}}{\partial \xi_{2}}=0 \quad \text { on } \Sigma \\
\frac{\partial v_{1,1}}{\partial \xi_{1}}\left(\xi ; \pm \frac{\pi}{2}\right)=0 \quad \text { at } \xi_{1}= \pm \frac{\pi}{2} \\
v_{1,1} \sim-\alpha_{0}^{1} \quad \text { as } \rho \rightarrow 0
\end{array}\right.
$$

Obviously, the function

$$
v_{1,1}\left(\xi ; x_{1}\right) \equiv-\alpha_{0}^{1}\left(x_{1}\right)
$$

is a solution of (115).

We rewrite the asymptotics of the series (112) as $\xi_{2} \rightarrow+\infty$, taking the asymptotics of terms (32), (56), (103), (116) which we have already constructed and the equality $\alpha_{1}^{0}\left(x_{1}\right) \equiv 0$ into account; following the matching method we conclude that the outer expansion must be sought in the form

$$
u_{\varepsilon}(x)=u_{0}(x)+\sum_{j=1}^{+\infty} \varepsilon^{j} \ln a u_{j, 1}+\sum_{j=1}^{+\infty} \varepsilon^{j} u_{j}(x)+\cdots,
$$

where $u_{1,1} \sim-\alpha_{0}^{1}$ as $x_{2} \rightarrow 0$. We seek the functions $u_{j, 1}$ in $C^{\infty}(\bar{\Omega})$; therefore this asymptotic behaviour is equivalent to the boundary conditions

$$
u_{1,1}\left(x_{1}, 0\right)=-\alpha_{0}^{1}\left(x_{1}\right), \quad x_{1} \in\left(-\frac{\pi}{2}, \frac{\pi}{2}\right) .
$$


Corresponding to (117), we seek an asymptotic expansion of the eigenvalue $\lambda_{\varepsilon}$ in the form

$$
\lambda_{\varepsilon}=\lambda_{0}+\sum_{j=1}^{+\infty} \varepsilon^{j} \ln a \lambda_{j, 1}+\sum_{j=1}^{+\infty} \varepsilon^{j} \lambda_{j}+\cdots
$$

By substituting the expansion (117) for $u_{\varepsilon}$ in terms of the variables $x$ and the expansion (119) into the problem and collecting terms in the powers $\varepsilon \ln a$, we obtain the following problem for $u_{1,1}$ :

$$
\left\{\begin{aligned}
-\Delta u_{1,1} & =\lambda_{0} u_{1,1}+\lambda_{1,1} u_{0} \quad \text { for } x \in \Omega, \\
u_{1,1} & =-\alpha_{0}^{1} \quad \text { for } x \in \Gamma_{4}, \\
\frac{\partial u_{1,1}}{\partial \nu} & =0 \quad \text { for } x \in \Gamma_{1} \cup \Gamma_{2} \cup \Gamma_{3} .
\end{aligned}\right.
$$

Correspondingly, collecting terms for the corresponding powers $\varepsilon^{j} \ln a(j>1)$ in the equation and boundary conditions, based on the same considerations as in Remark 3.1, we obtain the following problem for $u_{j, 1}$ :

$$
\left\{\begin{aligned}
-\Delta u_{j, 1} & =\lambda_{0} u_{j, 1}+\cdots+\lambda_{j-1} u_{1,1}+\lambda_{1,1} u_{j-1}+\cdots+\lambda_{j, 1} u_{0} \quad \text { for } x \in \Omega, \\
u_{j, 1} & =\alpha_{j, 1}^{0} \quad \text { for } x \in \Gamma_{4}, \\
\frac{\partial u_{j, 1}}{\partial \nu} & =0 \quad \text { for } x \in \Gamma_{1} \cup \Gamma_{2} \cup \Gamma_{3},
\end{aligned}\right.
$$

where the $\alpha_{j, 1}^{0}\left(x_{1}\right)$ are, for the moment, unknown (arbitrary) functions, whose odd derivatives vanish at $x_{1}= \pm \pi / 2$.

We now obtain an explicit formula for $\lambda_{1,1}$. We multiply the equation by $u_{0}$, integrate over the domain $\Omega$ taking the normalization of $u_{0}$ into account, and apply Green's formula twice to the left-hand side of the equality. Finally we obtain

$$
\lambda_{1,1}=\int_{-\frac{\pi}{2}}^{\frac{\pi}{2}} \alpha_{0}^{12}\left(x_{1}\right) d x_{1}>0 \quad\left(\text { or } \quad \lambda_{1,1}=\int_{\Gamma_{4}}\left(\frac{\partial u_{0}}{\partial \nu}\right)^{2} d s>0\right) .
$$

So that problems (120) and (121) are uniquely solvable, we choose all solutions to be orthogonal to $u_{0}$ in $L_{2}(\Omega)$.

We expand the functions $u_{j, 1}(x)$ in a Taylor series in the variable $x_{2}$ as $x_{2} \rightarrow 0$. We make the change of variables $\xi_{2}=x_{2} / \varepsilon$ (that is, we rewrite these expansions in the 'intermediate' variables). Then the asymptotic expression of the series (117) as $\varepsilon \xi_{2} \rightarrow 0$ has the form

$$
V_{k, 1}\left(\xi_{2} ; x_{1}\right)+\sum_{k=1}^{\infty} \varepsilon^{k} V_{k}\left(\xi_{2} ; x_{1}\right)+\cdots,
$$

where the $V_{k}\left(\xi_{2} ; x_{1}\right)$ are already known, while the $V_{k, 1}\left(\xi_{2} ; x_{1}\right)$ are polynomials of order $k-1$ in the variable $\xi_{2}$, with coefficients that are infinitely differentiable functions of $x_{1}$ on $[-\pi / 2, \pi / 2]$, whose odd derivatives vanish at $x_{1}= \pm \pi / 2$. We have the representation

$$
V_{k, 1}\left(\xi_{2} ; x_{1}\right)=\widetilde{V}_{k, 1}\left(\xi_{2} ; x_{1}\right)+\alpha_{k, 1}^{0}\left(x_{1}\right)
$$

and the $\widetilde{V}_{k, 1}$ are independent of $\alpha_{j, 1}^{0}, j \geq k$. In this notation, $\alpha_{1,1}^{0} \equiv-\alpha_{0}^{1}$ and $\widetilde{V}_{1,1} \equiv 0$. By the boundary-value problems (120), (121) and the definition of the $V_{q, 1}$, we have the 
following equalities:

$$
\begin{aligned}
&-\Delta_{\xi} V_{q, 1}= 2 \frac{\partial^{2} V_{q-1,1}}{\partial x_{1} \partial \xi_{1}}+\frac{\partial^{2} V_{q-2,1}}{\partial x_{1}^{2}}+\lambda_{0} V_{q-2,1}+\cdots \\
&+\lambda_{q-3} V_{1,1}+\lambda_{1,1} V_{q-3}+\cdots+\lambda_{q-3,1} V_{1}, \\
& \frac{\partial V_{q, 1}}{\partial x_{1}}\left(\xi_{2} ; \pm \frac{\pi}{2}\right)=0, \quad \frac{\partial V_{q, 1}}{\partial \xi_{1}}\left(\xi_{2} ; x_{1}\right) \equiv 0 .
\end{aligned}
$$

We point out that, as before, the condition

$$
\frac{d^{2 j+1} \alpha_{k, 1}^{0}}{d x_{1}^{2 j+1}}\left( \pm \frac{\pi}{2}\right)=0, \quad j=0,1, \ldots, \quad k=2,3, \ldots
$$

is important and we shall keep an eye on its validity.

Taking (123) and (117) into account and following the method of matched asymptotic expansions, we see that the intermediate expansion must have the structure

$$
u_{\varepsilon}(x)=\sum_{k=1}^{+\infty} \varepsilon^{k} \ln a v_{k, 1}\left(\frac{x}{\varepsilon} ; x_{1}\right)+\sum_{j=1}^{+\infty} \varepsilon^{j} v_{j}\left(\frac{x}{\varepsilon} ; x_{1}\right)+\cdots,
$$

where

$$
v_{q, 1}\left(\xi ; x_{1}\right)=V_{q, 1}\left(\xi_{2} ; x_{1}\right)+o(1) \quad \text { as } \quad \xi_{2} \rightarrow+\infty .
$$

In what follows we construct the coefficients $v_{k, 1}$ in the form $v_{k, 1}=V_{k, 1}+\widetilde{v}_{k, 1}$, where $\widetilde{v}_{k, 1} \in \mathbb{A}^{(k-1,1)}$. Substituting the series (128) and the expansion (122) into the problem (2) (first having rewritten it in terms of $\xi$; see (20) ) and collecting terms in $\varepsilon^{k-2} \ln a$ in the equation, and in $\varepsilon^{k-1} \ln a$ in the boundary conditions, using (129) we conclude that the $v_{k, 1}$ satisfy problems of the form

$$
\left\{\begin{aligned}
& \Delta_{\xi} v_{k, 1}= 2 \frac{\partial^{2} v_{k-1,1}}{\partial x_{1} \partial \xi_{1}}+\frac{\partial^{2} v_{k-2,1}}{\partial x_{1}^{2}}+\lambda_{0} v_{k-2,1}+\cdots+\lambda_{k-3} v_{1,1} \\
&+\lambda_{1,1} v_{k-3}+\cdots+\lambda_{k-3,1} v_{1} \quad \text { in } \Pi, \\
& \frac{\partial v_{k, 1}}{\partial \xi_{2}}=0 \quad \text { on } \Sigma \\
& \frac{\partial v_{k, 1}}{\partial \xi_{1}}\left(\xi ; \pm \frac{\pi}{2}\right)=-\frac{\partial v_{k-1,1}}{\partial x_{1}}\left(\xi ; \pm \frac{\pi}{2}\right) \quad \text { at } \xi_{1}= \pm \frac{\pi}{2} \\
& v_{k, 1}=V_{k, 1}+o(1) \quad \text { as } \xi_{2} \rightarrow+\infty
\end{aligned}\right.
$$

Rewriting the problem for $k=2$, taking (116) and (127) into account, we obtain

$$
\left\{\begin{array}{l}
-\Delta_{\xi} v_{2,1}=0 \quad \text { in } \Pi, \\
\frac{\partial v_{2,1}}{\partial \xi_{2}}=0 \quad \text { on } \Sigma, \\
\frac{\partial v_{2,1}}{\partial \xi_{1}}\left(\xi ; \pm \frac{\pi}{2}\right)=0 \quad \text { at } \xi_{1}= \pm \frac{\pi}{2} \\
v_{2,1}=\alpha_{1,1}^{1} \xi_{2}+\alpha_{2,1}^{0} \quad \text { as } \xi_{2} \rightarrow+\infty .
\end{array}\right.
$$

By Theorem 3.1

$$
v_{2,1}=V_{2,1}+\widetilde{v}_{2,1}
$$

is a solution of problem (131), where $\widetilde{v}_{2,1} \in \mathbb{A}^{(1,1)}$ is a completely defined function that is independent of $\alpha_{2,1}^{0}$. 
In view of (132), the asymptotics of this function at zero (as $\rho \rightarrow 0$ ) look like:

$$
v_{2,1}\left(\xi ; x_{1}\right)=\mathcal{M}_{2,1}\left(x_{1}\right) \ln \rho+\widehat{\mathcal{M}}_{2,1}\left(x_{1}\right)+\alpha_{2,1}^{0}\left(x_{1}\right)+O(\rho) .
$$

We rewrite the asymptotics of $\varepsilon^{2} \ln a v_{2,1}\left(\xi ; x_{1}\right)$ as $\rho \rightarrow 0$ in terms of $\zeta$, taking (133) into account. As $a \tau \rightarrow 0$,

$$
\begin{aligned}
\varepsilon^{2} \ln a v_{2,1}\left(a \zeta ; x_{1}\right)= & \varepsilon^{2} \ln a \mathcal{M}_{2,1}\left(x_{1}\right)[\ln a+\ln \tau]+\varepsilon^{2} \ln a \widehat{\mathcal{M}}_{2,1}\left(x_{1}\right) \\
& +\varepsilon^{2} \ln a \alpha_{2,1}^{0}\left(x_{1}\right)+O\left(\varepsilon^{2} \ln a a^{2} \tau^{2}\right)
\end{aligned}
$$

Rewriting the asymptotics of the series (128) as $\rho \rightarrow 0$ in terms of $\zeta$, taking (134) into account, and following the method of matched asymptotic expansions, we conclude that in (114) as $\tau \rightarrow+\infty$ the term $w_{2,1,0}$ has asymptotics of the form

$$
w_{2,1,0} \sim \mathcal{M}_{2,1} \ln \tau+\widehat{\mathcal{M}}_{2,1}+\mathcal{M}_{2}+\alpha_{2,1}^{0} \text {. }
$$

Taking the form of equation (40) into account in the inner variables $\zeta$, we substitute the expansion (114) and the series (119) into the problem (2) and collect terms with like powers of $\varepsilon, a$ and $\ln a$ (in the equation with $a^{-2} \ln a$, and in the boundary conditions, with $\varepsilon a^{-1} \ln a$, respectively). In view of (135) we obtain a problem for the function $w_{2,1,0}$ of the form

$$
\left\{\begin{array}{l}
\Delta_{\zeta} w_{2,1,0}=0 \quad \text { in } \mathbb{R}_{+}^{2} \\
\frac{\partial w_{2,1,0}}{\partial \zeta_{2}}=0 \quad \text { on } \Gamma \\
w_{2,1,0}=0 \quad \text { on } \gamma \\
w_{2,1,0} \sim \mathcal{M}_{2,1} \ln \tau+\widehat{\mathcal{M}}_{2,1}+\mathcal{M}_{2}+\alpha_{2,1}^{0} \quad \text { as } \tau \rightarrow+\infty
\end{array}\right.
$$

Taking into account the form of the asymptotics (44) and problems (43), we conclude that the function

$$
w_{2,1,0}\left(\zeta ; x_{1}\right)=\mathcal{M}_{2,1}\left(x_{1}\right) Y(\zeta)
$$

is a solution of problem (136) for

$$
\alpha_{2,1}^{0}\left(x_{1}\right) \equiv \mathcal{M}_{2,1} \ln 2-\widehat{\mathcal{M}}_{2,1}-\mathcal{M}_{2}
$$

Therefore we fix $w_{2,1,0}$ and $\alpha_{2,1}^{0}$ in accordance with (137) and (138). Then the function $v_{2,1}$ is uniquely determined.

From (121), solved for $u_{2,1}$, we find $\lambda_{2,1}$ and determine $u_{2,1}$.

It is easy to show that the following assertion is true, similarly to Lemma 3.3 .

Lemma 3.4. a) Let

$$
V\left(\xi ; x_{1}\right)=\sum_{k=1}^{\infty} \varepsilon^{k} v_{k}\left(\xi ; x_{1}\right)+\sum_{k=1}^{\infty} \varepsilon^{k} \ln a v_{k, 1}\left(\xi ; x_{1}\right)
$$

where $v_{k, 1}=\mathcal{P}_{k-1}+\widetilde{v}_{k, 1}, \quad \widetilde{v}_{k, 1} \in \mathbb{A}^{(k-1,1)}, \quad k \geq 2, \quad v_{1,1}=\check{\mathcal{M}}_{1,1}$, and the $v_{k}$ satisfy the assertions of Theorem 3.2 . Then the asymptotics of the series $V$ as $\rho \rightarrow 0$, rewritten in 
terms of $\zeta$, can be represented in the form

$$
\begin{aligned}
V\left(\xi ; x_{1}\right)= & \ln ^{2} a \sum_{k=2}^{\infty} \varepsilon^{k} \mathcal{M}_{k, 1}\left(x_{1}\right)+\ln a \varepsilon\left(\check{\mathcal{M}}_{1,1}\left(x_{1}\right)+\mathcal{M}_{1}\left(x_{1}\right)\right) \\
& +\ln a \sum_{k=2}^{\infty} \varepsilon^{k}\left(\mathcal{M}_{k, 1}\left(x_{1}\right) \ln \tau+\check{\mathcal{M}}_{k, 1}\left(x_{1}\right)+\mathcal{M}_{k}\left(x_{1}\right)\right) \\
& +\sum_{k=1}^{\infty} \varepsilon^{k} \mathcal{M}_{k}\left(x_{1}\right)(\ln \tau+\ln 2)+O(a \tau \ln (a \tau)) \quad \text { as } \quad \text { a } \rightarrow 0
\end{aligned}
$$

where the coefficients $\mathcal{M}_{k}\left(x_{1}\right)$ and $\check{\mathcal{M}}_{k, 1}\left(x_{1}\right)$, which satisfy

$$
\frac{d^{2 j+1} \mathcal{M}_{k, 1}}{d x_{1}^{2 j+1}}\left( \pm \frac{\pi}{2}\right)=0, \quad \frac{d^{2 j+1} \check{\mathcal{M}}_{k, 1}}{d x_{1}^{2 j+1}}\left( \pm \frac{\pi}{2}\right)=0, \quad j=0, \ldots,
$$

are determined from the asymptotic behaviour of $\widetilde{v}_{k, 1}=\mathcal{M}_{k, 1} \ln \rho+\check{\mathcal{M}}_{k, 1}, \quad k \geq 2$, as $\rho \rightarrow 0$.

b) If in addition the $v_{j, 1}$ are solutions of the problems

$$
\left\{\begin{aligned}
& \Delta_{\xi} v_{k, 1}= 2 \frac{\partial^{2} v_{k-1,1}}{\partial x_{1} \partial \xi_{1}}+\frac{\partial^{2} v_{k-2,1}}{\partial x_{1}^{2}}+\lambda_{0} v_{k-2,1}+\cdots+\lambda_{k-3} v_{1,1} \\
&+\lambda_{1,1} v_{k-3}+\cdots+\lambda_{k-3,1} v_{1} \quad \text { in } \Pi, \\
& \frac{\partial v_{k, 1}}{\partial \xi_{2}}=0 \quad \text { on } \Sigma \\
& \frac{\partial v_{k, 1}}{\partial \xi_{1}}\left(\xi ; \pm \frac{\pi}{2}\right)=0 \quad \text { at } \xi_{1}= \pm \frac{\pi}{2} \\
& v_{k, 1}=V_{k, 1}+o(1) \quad \text { as } \xi_{2} \rightarrow+\infty
\end{aligned}\right.
$$

then the $v_{j, 1}$ are solutions of the problems (130).

We again seek the terms of the intermediate expansion $v_{k, 1}$ that are solutions of (141) and, consequently, by part b) of Lemma 3.4, also solutions of (130). Notice that the functions $v_{1,1}$ and $v_{2,1}$ constructed earlier possess these properties.

Following the method of matched asymptotic expansions, by part a) of Lemma 3.4. from the representation (139) we conclude that the inner expansion must be sought in the form

$$
u_{\varepsilon}=\sum_{k=2}^{\infty} \varepsilon^{k} \ln ^{2} a w_{k, 2,0}+\sum_{k=2}^{\infty} \varepsilon^{k} \ln a w_{k, 1,0}+\sum_{k=1}^{\infty} \varepsilon^{k} w_{k, 0,0}+\cdots,
$$

where, as $\tau \rightarrow+\infty$, the terms $w_{k, 1,0}$ have asymptotics of the form

$$
w_{k, 1,0} \sim \mathcal{M}_{k, 1} \ln \tau+\check{\mathcal{M}}_{k, 1}+\mathcal{M}_{k}
$$

and the term $w_{2,2,0}$ has asymptotics of the form

$$
w_{2,2,0} \sim \mathcal{M}_{2,1} .
$$

Completely similarly to Theorem 3.2 , taking Lemma 3.4 (instead of Lemma 3.3) into account, one can prove the following assertion.

Theorem 3.3. There exist series (117), (119), (128) and (142) such that

a) the coefficients $\lambda_{k}, u_{k}, v_{k}$ and $w_{k, 0,0}$ satisfy the assertions of Theorem 3.2;

b) the pairs $u_{j, 1} \in C^{\infty}(\bar{\Omega})$ and $\lambda_{j, 1}$ are solutions of problems (121);

c) the coefficient $\lambda_{1,1}$ is determined from formula (122); 
d) the functions $v_{j, 1}$ can be represented as the sums $v_{j, 1}=V_{j, 1}+\widetilde{v}_{j, 1}, \widetilde{v}_{j, 1} \in \mathbb{A}^{(j-1,1)}$, $v_{1,1}=-\mathcal{M}_{1}$, and are solutions of problems (130);

e) in terms of the variables $\zeta$, as $\rho \rightarrow 0$ the series (128) has asymptotics of the form

$$
\begin{aligned}
u_{\varepsilon}= & \ln ^{2} a \sum_{k=2}^{\infty} \varepsilon^{k} \mathcal{M}_{k, 1}\left(x_{1}\right) \\
& +\ln a \sum_{k=2}^{\infty} \varepsilon^{k} \mathcal{M}_{k, 1}\left(x_{1}\right)(\ln \tau+\ln 2) \\
& +\sum_{k=1}^{\infty} \varepsilon^{k} \mathcal{M}_{k}\left(x_{1}\right)(\ln \tau+\ln 2) \\
& +O(a \tau \ln (a \tau)) \quad \text { as } \quad a \tau \rightarrow 0,
\end{aligned}
$$

where

$$
\frac{d^{2 j+1} \mathcal{M}_{k, 1}}{d x_{1}^{2 j+1}}\left( \pm \frac{\pi}{2}\right)=0, \quad j=0,1, \ldots
$$

f) the summands $w_{k, 1,0}=\mathcal{M}_{k, 1} Y$ are solutions of the problems

$$
\left\{\begin{aligned}
\Delta_{\zeta} w_{k, 1,0} & =0 \quad \text { in } \mathbb{R}_{+}^{2}, \\
\frac{\partial w_{k, 1,0}}{\partial \zeta_{2}} & =0 \quad \text { on } \Gamma \\
w_{k, 1,0} & =0 \quad \text { on } \gamma, \\
w_{k, 1,0} & \sim \mathcal{M}_{k, 1}(\ln \tau+\ln 2) \quad \text { as } \tau \rightarrow+\infty .
\end{aligned}\right.
$$

The succeeding terms in the expansions in terms of powers of $\ln a$ are also constructed completely similarly. Moreover, the algorithm to construct the terms $u_{k, 2}, \lambda_{k, 2}, v_{k, 2}$, and $w_{k, 2,0}$ of the corresponding series and to eliminate $w_{2,2,0}$ repeats the algorithm used to construct the terms $u_{k, 1}, \lambda_{k, 1}, v_{k, 1}$, and $w_{k, 1,0}$ of the series (117), (119), (128), and (142), respectively, and to eliminate $w_{1,1,0}$. In a similar fashion, the term $w_{3,3,0}$ appears, which plays the same role as the terms $w_{1,1,0}$ and $w_{2,2,0}$. To go further we repeat our arguments.

Indeed, by formally rewriting the problem for the term $w_{2,2,0}$ with the asymptotics (144) we verify that this problem has no solutions. As before, we introduce in the intermediate expansion the term $v_{2,2} \equiv-\mathcal{M}_{2,1}\left(x_{1}\right)$, which is independent of $\xi$ and plays the same role as $v_{1,1}$ given in (116). Then we find the terms $\lambda_{2,2}$ and $u_{2,2}$, which play exactly the same role as $\lambda_{1,1}$ and $u_{1,1}$. Next, by rewriting the asymptotics of the intermediate expansion as $\rho \rightarrow 0$, including these new terms, in terms of the variables $\zeta$, we conclude that the term $w_{3,3,0}$ appears in the inner expansion, which plays the same role as $w_{1,1,0}$ and $w_{2,2,0}$. The rest of the procedure to construct the terms and eliminate $w_{3,3,0}$ is completely similar.

Thus, by continuing the process of constructing the terms of the expansions, we show that we have the next result. We set $\lambda_{k, 0}:=\lambda_{k}, u_{k, 0}:=u_{k}, v_{k, 0}:=v_{k}(k \geq 1)$ and $\lambda_{0,0}:=\lambda_{0}, \quad u_{0,0}:=u_{0}, \quad v_{0,0} \equiv 0$. We have the following theorem. 
Theorem 3.4. There exist series

$$
\begin{aligned}
u_{\varepsilon}(x) & =\sum_{k=0}^{+\infty} \sum_{j=k}^{+\infty} \varepsilon^{j} \ln ^{k} a u_{j, k}(x), \\
\lambda_{\varepsilon} & =\sum_{k=0}^{+\infty} \sum_{j=k}^{+\infty} \varepsilon^{j} \ln ^{k} a \lambda_{j, k}, \\
u_{\varepsilon}(x) & =\sum_{k=0}^{+\infty} \sum_{j=k}^{+\infty} \varepsilon^{j} \ln ^{k} a v_{j, k}\left(\xi ; x_{1}\right), \quad v_{0,0} \equiv 0, \\
u_{\varepsilon}(x) & =\sum_{k=0}^{+\infty} \sum_{j=k+1}^{+\infty} \varepsilon^{j} \ln ^{k} a w_{j, k, 0}\left(\zeta ; x_{1}\right),
\end{aligned}
$$

such that

a) the pairs $u_{j, k} \in C^{\infty}(\bar{\Omega})$ and $\lambda_{j, k}$ are solutions of the problems

$$
\left\{\begin{aligned}
-\Delta u_{j, k} & =\sum_{p=0}^{j} \sum_{q=p}^{k+j-p} \lambda_{p, q} u_{j-p, k-q} \quad \text { in } \Omega, \\
\frac{\partial u_{j, k}}{\partial \nu} & =0 \quad \text { on } \Gamma_{1} \cup \Gamma_{2} \cup \Gamma_{3}, \\
u_{j, k} & =\alpha_{j, k}^{0} \quad \text { on } \Gamma_{4} ;
\end{aligned}\right.
$$

b) the series (148) has the following asymptotics as $x_{2} \rightarrow 0$, rewritten in terms of the variables $\xi$ :

$$
u_{\varepsilon}(x)=\sum_{k=0}^{+\infty} \sum_{j=k}^{+\infty} \varepsilon^{j} \ln ^{k} a V_{j, k}\left(\xi_{2} ; x_{1}\right)+\cdots,
$$

where the $V_{j, k}\left(\xi_{2} ; x_{1}\right)$ are polynomials of order $j-k$ in the variable $\xi_{2}$ whose coefficients are infinitely differentiable functions of $x_{1}$ on $[-\pi / 2, \pi / 2]$ and whose odd derivatives vanish at $x_{1}= \pm \pi / 2$;

c) the polynomials $V_{j, k}$ satisfy the equalities

$$
\begin{gathered}
-\Delta_{\xi} V_{j, k}=2 \frac{\partial^{2} V_{j-1, k}}{\partial x_{1} \partial \xi_{1}}+\frac{\partial^{2} V_{j-2, k}}{\partial x_{1}^{2}}+\sum_{p=0}^{j-2} \sum_{q=p}^{k+j-p} \lambda_{p, q} V_{j-2-p, k-q}, \\
\frac{\partial V_{j, k}}{\partial x_{1}}\left(\xi_{2} ; \pm \frac{\pi}{2}\right)=0, \quad \frac{\partial V_{j, k}}{\partial \xi_{1}}\left(\xi_{2} ; x_{1}\right) \equiv 0 ;
\end{gathered}
$$

d) the coefficient $\lambda_{1,1}$ is determined from formula (122);

e) the functions $v_{j, k}$ can be represented as the sums $v_{j, k}=V_{j, k}+\widetilde{v}_{j, k}, \widetilde{v}_{j, k} \in \mathbb{A}^{(j-k, 1)}$, $v_{j, j}=\mathcal{M}_{j, j}$, and are solutions of the problems

$$
\left\{\begin{aligned}
&-\Delta_{\xi} v_{j, k}= 2 \frac{\partial^{2} v_{j-1, k}}{\partial x_{1} \partial \xi_{1}}+\frac{\partial^{2} v_{j-2, k}}{\partial x_{1}^{2}} \\
&+\sum_{p=0}^{j-2} \sum_{q=p}^{k+j-p} \lambda_{p, q} v_{j-2-p, k-q} \quad \text { in } \Pi \\
& \frac{\partial v_{j, k}}{\partial \xi_{2}}=0 \quad \text { on } \Sigma \\
& \frac{\partial v_{j, k}}{\partial \xi_{1}}\left(\xi ; \pm \frac{\pi}{2}\right)=0 \quad \text { at } \xi_{1}= \pm \frac{\pi}{2}
\end{aligned}\right.
$$


f) as $\rho \rightarrow 0$ the series (150), rewritten in terms of $\zeta$, has asymptotics of the form

$$
u_{\varepsilon}=\sum_{k=0}^{+\infty} \sum_{j=k}^{+\infty} \varepsilon^{j} \ln ^{k} a \mathcal{M}_{j, k}\left(x_{1}\right)(\ln \tau+\ln 2)+O(a \tau \ln (a \tau)), \quad \text { as a } \rightarrow 0,
$$

where

$$
\frac{d^{2 l+1} \mathcal{M}_{k, j}}{d x_{1}^{2 l+1}}\left( \pm \frac{\pi}{2}\right)=0, \quad l=0,1, \ldots ;
$$

g) the summands $w_{k, j, 0}=\mathcal{M}_{k, j} Y$ are solutions of the problems

$$
\left\{\begin{aligned}
\Delta_{\zeta} w_{k, j, 0} & =0 \quad \text { in } \mathbb{R}_{+}^{2}, \\
\frac{\partial w_{k, j, 0}}{\partial \zeta_{2}} & =0 \quad \text { on } \Gamma \\
w_{k, j, 0} & =0 \quad \text { on } \gamma \\
w_{k, j, 0} & \sim \mathcal{M}_{k, j}(\ln \tau+\ln 2) \quad \text { as } \tau \rightarrow+\infty .
\end{aligned}\right.
$$

We point out that if there are negative limits of summation in problems (152) and (158) or later in the paper, this means that these sums do not appear; similarly, terms with 'smaller' indices, that have not so far appeared, are assumed to be identically zero.

3.3. The construction of the asymptotics. Third step. We now determine the asymptotics (33) more precisely, using (30), the asymptotics (55), the asymptotic behaviour of the function $X_{2}$ in Lemma 3.1, and the consequent asymptotics of the terms occurring in the intermediate expansion.

Due to parts e) and f) of Theorem 3.4, rewriting the asymptotics of the series (150) as $\rho \rightarrow 0$ in terms of $\zeta$ we obtain

$$
\begin{aligned}
& u_{\varepsilon}=\sum_{k=0}^{+\infty} \sum_{j=k}^{+\infty} \varepsilon^{j} \ln ^{k} a W_{j, k, 0}\left(\zeta ; x_{1}\right)+\sum_{k=0}^{+\infty} \sum_{j=k}^{+\infty} a \varepsilon^{j} \ln ^{k} a W_{j, k, 1}\left(\zeta ; x_{1}\right) \\
& \quad+O\left(a^{2} \tau^{2} \ln (a \tau)\right) \quad \text { as } \quad a \tau \rightarrow 0, \\
& W_{j, k, 0}\left(\zeta ; x_{1}\right)=\mathcal{M}_{j, k}\left(x_{1}\right)(\ln \tau+\ln 2), \quad j \geq k, \\
& W_{j, k, 1}\left(\zeta ; x_{1}\right)=\left(N_{j, k, 1}\left(x_{1}\right) \tau \ln \tau \cos \theta+\widehat{N}_{j, k, 1}\left(x_{1}\right) \tau \cos \theta\right), \quad j \geq k+1, \\
& W_{k, k, 1}\left(\zeta ; x_{1}\right)=\widehat{N}_{k, k, 1}\left(x_{1}\right) \tau \cos \theta, \\
& W_{0,0,1} \equiv 0, \quad W_{1,0,1} \equiv 0, \quad W_{1,1,1} \equiv 0, \quad N_{2,1,1} \equiv 0, \quad W_{2,2,1} \equiv 0 .
\end{aligned}
$$

We observe that using the boundary-value problems (155) and the definition of the $W_{j, k, l}$, the following equality holds:

$$
-\Delta_{\zeta} W_{j, k, 1}=2 \frac{\partial^{2} W_{j-1, k, 0}}{\partial x_{1} \partial \zeta_{1}}
$$

and by the definition of the class $\mathbb{A}^{(k, q)}$ we have

$$
\frac{\partial^{2 i} N_{j, k, 1}}{\partial x_{1}^{2 i}}\left(\xi ; \pm \frac{\pi}{2}\right)=0, \quad \frac{\partial^{2 i} \widehat{N}_{j, k, 1}}{\partial x_{1}^{2 i}}\left(\xi ; \pm \frac{\pi}{2}\right)=0, \quad i=0,1, \ldots
$$

and, consequently,

$$
\frac{\partial^{2 i} W_{j, k, 1}}{\partial x_{1}^{2 i}}\left(\xi ; \pm \frac{\pi}{2}\right)=0, \quad i=0,1, \ldots
$$


Following the method of matched asymptotic expansions, by (159) we conclude that the inner expansion must be sought in the form

$$
u_{\varepsilon}(x)=\sum_{k=0}^{+\infty} \sum_{j=k+1}^{+\infty} \varepsilon^{j} \ln ^{k} a w_{j, k, 0}\left(\zeta ; x_{1}\right)+\sum_{k=0}^{+\infty} \sum_{j=k+1}^{+\infty} a \varepsilon^{j} \ln ^{k} a w_{j, k, 1}\left(\zeta ; x_{1}\right)+\cdots
$$

where $w_{0,0,1} \equiv 0, w_{1,0,1} \equiv 0, w_{1,1,1} \equiv 0, w_{2,2,1} \equiv 0$.

We rewrite the operator in problem (2) in terms of the inner variables $\zeta$, substitute the expansion (163) and the series (149) into (2), and collect the terms with the powers $a^{-1} \varepsilon^{j-2} \ln ^{k} a$ in the equation, and $\varepsilon^{j-1} \ln ^{k} a$ in the boundary conditions. We obtain a problem for the functions $w_{j, k, 1}$ of the form

$$
\left\{\begin{aligned}
&-\Delta_{\zeta} w_{j, k, 1}=2 \frac{\partial^{2} w_{j-1, k, 0}}{\partial x_{1} \partial \zeta_{1}} \quad \text { in } \mathbb{R}_{+}^{2}, \\
& \frac{\partial w_{j, k, 1}}{\partial \zeta_{2}}=0 \quad \text { on } \Gamma \\
& w_{j, k, 1}=0 \quad \text { on } \gamma \\
& w_{j, k, 1} \sim W_{j, k, 1} \quad \text { as } \tau \rightarrow+\infty .
\end{aligned}\right.
$$

We introduce into consideration the auxiliary problem

$$
\left\{\begin{array}{l}
-\Delta_{\zeta} Y_{1}=2 \frac{\partial Y}{\partial \zeta_{1}} \quad \text { in } \mathbb{R}_{+}^{2}, \\
\frac{\partial Y_{1}}{\partial \zeta_{2}}=0 \quad \text { on } \Gamma, \\
Y_{1}=0 \quad \text { on } \gamma, \\
Y_{1} \sim \tau \ln \tau \cos \theta \quad \text { as } \tau \rightarrow+\infty .
\end{array}\right.
$$

We observe that a solution $Y_{1} \in H_{\text {loc }}^{1}\left(\mathbb{R}_{+}^{2}\right)$ of (165) can be represented in the form

$$
Y_{1}(\zeta)=\zeta_{1} Y(\zeta)
$$

We consider the following function $Y_{2}$ (see [73]):

$$
Y_{2}(\zeta)=\operatorname{Re} \sqrt{y^{2}-1}
$$

where $y=\zeta_{1}+\mathrm{i} \zeta_{2}$. It is easy to verify that $Y_{2} \in H_{\text {loc }}^{1}\left(\mathbb{R}_{+}^{2}\right)$ is a solution of the problem

$$
\left\{\begin{array}{l}
-\Delta_{\zeta} Y_{2}=0 \quad \text { in } \mathbb{R}_{+}^{2}, \\
\frac{\partial Y_{2}}{\partial \zeta_{2}}=0 \quad \text { on } \Gamma \\
Y_{2}=0 \quad \text { on } \gamma \\
Y_{2} \sim \tau \cos \theta \quad \text { as } \tau \rightarrow+\infty
\end{array}\right.
$$

It follows from the explicit form of the functions $Y_{1}$ and $Y_{2}$ that

$$
\begin{aligned}
Y_{1}(\zeta)= & \tau(\ln \tau+\ln 2) \cos \theta+\sum_{j=0}^{\infty} C_{2 j+1} \tau^{-2 j-1}(\cos ((2 j+1) \theta) \\
& +\cos ((2 j+3) \theta)) \\
Y_{2}(\zeta)= & \tau \cos \theta+\sum_{j=0}^{+\infty} c_{2 j+1} \tau^{-2 j-1} \cos ((2 j+1) \theta) \quad \text { as } \tau \rightarrow+\infty
\end{aligned}
$$

Now, equation (160) implies that

$$
-N_{j, k, 1}\left(x_{1}\right)=\mathcal{M}_{j, k}^{\prime}\left(x_{1}\right)
$$


Taking into account the asymptotics (169) and problems (165), (168), from equality (170) we conclude that the function

$$
w_{j, k, 1}\left(\zeta ; x_{1}\right)=N_{j, k, 1}\left(x_{1}\right) Y_{1}(\zeta)+\left(\widehat{N}_{j, k, 1}\left(x_{1}\right)-N_{j, k, 1}\left(x_{1}\right) \ln 2\right) Y_{2}(\zeta)
$$

is a solution of problem (164).

Thus, we have proved the following theorem.

Theorem 3.5. There exist series (148), (149), (150), and (163) such that

a) the coefficients $\lambda_{j, k, 0}=\lambda_{j, k}, u_{j, k, 0} \equiv u_{j, k}, v_{j, k, 0} \equiv v_{j, k}, w_{j, k, 0}$ satisfy the assertions of Theorem 3.4;

b) the asymptotics of the series (150) as $\rho \rightarrow 0$, rewritten in terms of the variables $\zeta$, have the form (159), where the $W_{j, k, 1}$ satisfy (162);

c) the terms of the series $w_{j, k, 0}$ satisfy the equalities

$$
w_{j, k, 0}=W_{j, k, 0}+O\left(\tau^{-2}\right) \quad \text { as } \quad \tau \rightarrow+\infty ;
$$

d) the functions $w_{j, k, 1}$ are solutions of (164) and have the asymptotics

$$
w_{j, k, 1}=W_{j, k, 1}+O\left(\tau^{-1}\right) \quad \text { as } \quad \tau \rightarrow+\infty \text {; }
$$

e) the asymptotics of the series (163) as $\tau \rightarrow+\infty$, rewritten in terms of $\xi$, have the form

$$
u_{\varepsilon}=\sum_{k=0}^{+\infty} \sum_{j=k}^{+\infty} \varepsilon^{j} \ln ^{k} a \mathcal{V}_{j, k, 0}\left(\xi ; x_{1}\right)+O\left(a^{2} \rho^{-1}\right) \quad \text { as } \quad \rho a^{-1} \rightarrow+\infty ;
$$

f) the terms of the series $v_{j, k, 0}$ satisfy the equalities

$$
v_{j, k, 0}=\mathcal{V}_{j, k, 0}+O\left(\rho^{2} \ln \rho\right) \quad \text { as } \rho \rightarrow 0 .
$$

It follows from part e) of Theorem 3.5 that we do not need to introduce terms of order $a$ in the intermediate expansion (150) and, consequently, we do not need them either in the outer expansion (148), or in the expansion of the eigenvalues (149).

We will determine the asymptotic behaviour (159) again, more precisely. By parts e) and $\mathrm{f}$ ) of Theorem 3.4 we obtain

$$
\begin{aligned}
u_{\varepsilon}= & \sum_{k=0}^{+\infty} \sum_{j=k}^{+\infty} \varepsilon^{j} \ln ^{k} a W_{j, k, 0}\left(\zeta ; x_{1}\right)+\sum_{k=0}^{+\infty} \sum_{j=k}^{+\infty} a \varepsilon^{j} \ln ^{k} a W_{j, k, 1}\left(\zeta ; x_{1}\right) \\
+ & \sum_{k=0}^{+\infty} \sum_{j=k+1}^{+\infty} a^{2} \varepsilon^{j} \ln ^{k} a \widetilde{W}_{j, k, 2}\left(\zeta ; x_{1}\right)+O\left(a^{3} \tau^{3} \ln (a \tau)\right) \quad \text { as } \quad a \tau \rightarrow 0 \\
\widetilde{W}_{j, k, 2}\left(\zeta ; x_{1}\right)= & \left(\tau^{2} \ln \tau\left(N_{j, k, 2}^{1}\left(x_{1}\right)+N_{j, k, 2}^{2}\left(x_{1}\right) \cos 2 \theta\right)\right) \\
& +\tau^{2}\left(\widehat{N}_{j, k, 2}^{1}\left(x_{1}\right)+\widehat{N}_{j, k, 2}^{2}\left(x_{1}\right) \cos 2 \theta\right), \quad j \geq k+2, \\
\widetilde{W}_{k+1, k, 2}\left(\zeta ; x_{1}\right)= & \tau^{2}\left(\widehat{N}_{k+1, k, 2}^{1}\left(x_{1}\right)+\widehat{N}_{k+1, k, 2}^{2}\left(x_{1}\right) \cos 2 \theta\right), \\
\widetilde{W}_{1,0,2}\left(\zeta ; x_{1}\right)= & \widehat{N}_{1,0,2}^{2}\left(x_{1}\right) \tau^{2} \cos 2 \theta
\end{aligned}
$$

where the $W_{j, k, 0}$ and $W_{j, k, 1}$ satisfy (159). We observe that by the boundary-value problems (155) and the definition of the $W_{j, k, 0}, W_{j, k, 1}, \widetilde{W}_{j, k, 2}$ we have the following equality:

$$
-\Delta_{\zeta} \widetilde{W}_{j, k, 2}=2 \frac{\partial^{2} W_{j-1, k, 1}}{\partial x_{1} \partial \zeta_{1}}+\frac{\partial^{2} W_{j-2, k, 0}}{\partial x_{1}^{2}}+\sum_{p=0}^{j-2} \sum_{q=p}^{k+j-p} \lambda_{p, q, 0} W_{j-2-p, k-q, 0}
$$


and by the definition of the class $\mathbb{A}^{(k, q)}$ we have

$$
\begin{aligned}
& \frac{\partial^{2 i+1} N_{j, k, 2}^{1}}{\partial x_{1}^{2 i+1}}\left(\xi ; \pm \frac{\pi}{2}\right)=0, \quad \frac{\partial^{2 i+1} \widehat{N}_{j, k, 2}^{2}}{\partial x_{1}^{2 i+1}}\left(\xi ; \pm \frac{\pi}{2}\right)=0, \\
& \frac{\partial^{2 i+1} N_{j, k, 2}^{1}}{\partial x_{1}^{2 i+1}}\left(\xi ; \pm \frac{\pi}{2}\right)=0, \quad \frac{\partial^{2 i+1} \widehat{N}_{j, k, 2}^{2}}{\partial x_{1}^{2 i+1}}\left(\xi ; \pm \frac{\pi}{2}\right)=0, \quad i=0,1, \ldots
\end{aligned}
$$

and, consequently,

$$
\frac{\partial^{2 i+1} \widetilde{W}_{j, k, 2}}{\partial x_{1}^{2 i+1}}\left(\xi ; \pm \frac{\pi}{2}\right)=0, \quad i=0,1, \ldots
$$

Following the method of matched asymptotic expansions, by (176) we conclude that the inner expansion must be sought in the form

$$
\begin{aligned}
u_{\varepsilon}(x)= & \sum_{k=0}^{+\infty} \sum_{j=k}^{+\infty} \varepsilon^{j} \ln ^{k} a w_{j, k, 0}\left(\zeta ; x_{1}\right)+\sum_{k=0}^{+\infty} \sum_{j=k}^{+\infty} a \varepsilon^{j} \ln ^{k} a w_{j, k, 1}\left(\zeta ; x_{1}\right) \\
& +\sum_{k=0}^{+\infty} \sum_{j=k+1}^{+\infty} a^{2} \varepsilon^{j} \ln ^{k} a w_{j, k, 2}\left(\zeta ; x_{1}\right)+\cdots
\end{aligned}
$$

where $w_{0,0,1} \equiv 0, w_{1,0,1} \equiv 0, w_{1,1,1} \equiv 0, w_{2,2,1} \equiv 0$.

We rewrite the operator in problem (2) in terms of the inner variables $\zeta$ (see (40)), substitute the expansion (180) and the series (149) into (2), and collect terms in the powers $\varepsilon^{j-2} \ln ^{k} a$ in the equation, and $a \varepsilon^{j-1} \ln ^{k} a$ in the boundary conditions. We obtain problems for the functions $w_{j, k, 2}$ of the form

$$
\left\{\begin{aligned}
-\Delta_{\zeta} w_{j, k, 2}= & 2 \frac{\partial^{2} w_{j-1, k, 1}}{\partial x_{1} \partial \zeta_{1}}+\frac{\partial^{2} w_{j-2, k, 0}}{\partial x_{1}^{2}} \\
& +\sum_{p=0}^{j-2} \sum_{q=p}^{k+j-p} \lambda_{p, q} w_{j-2-p, k-q, 0} \quad \text { in } \mathbb{R}_{+}^{2}, \\
\frac{\partial w_{j, k, 2}}{\partial \zeta_{2}}=0 \quad \text { on } \Gamma, & \text { on } \gamma, \\
w_{j, k, 2}=0 & \widetilde{W}_{j, k, 2} \quad \text { as } \tau \rightarrow+\infty .
\end{aligned}\right.
$$

In particular, the problem for $w_{1,0,2}$ takes the form

$$
\left\{\begin{array}{l}
-\Delta_{\zeta} w_{1,0,2}=0 \quad \text { in } \mathbb{R}_{+}^{2} \\
\frac{\partial w_{1,0,2}}{\partial \zeta_{2}}=0 \quad \text { on } \Gamma \\
w_{1,0,2}=0 \quad \text { on } \gamma \\
w_{1,0,2} \sim \widehat{N}_{1,0,2}^{2} \tau^{2} \cos \theta \quad \text { as } \tau \rightarrow+\infty
\end{array}\right.
$$

From the explicit form of the function $X$ and its asymptotics (30) we see that the coefficient satisfies $\widehat{N}_{1,0,2}^{1} \equiv 0$. Consider the following function $Y_{3}$ (see [73]):

$$
Y_{3}(\zeta)=\operatorname{Re} y \sqrt{y^{2}-1}
$$


where $y=\zeta_{1}+\mathrm{i} \zeta_{2}$. It is easy to verify that $Y_{2} \in H_{\text {loc }}^{1}\left(\mathbb{R}_{+}^{2}\right)$ is a solution of

$$
\left\{\begin{array}{l}
\Delta_{\zeta} Y_{3}=0 \quad \text { in } \mathbb{R}_{+}^{2}, \\
\frac{\partial Y_{3}}{\partial \zeta_{2}}=0 \text { on } \Gamma, \\
Y_{3}=0 \text { on } \gamma, \\
Y_{3} \sim \tau^{2} \cos 2 \theta \quad \text { as } \tau \rightarrow+\infty .
\end{array}\right.
$$

It follows from the explicit form of the function $Y_{3}$ that

$$
Y_{3}(\zeta)=\tau^{2} \cos 2 \theta-\frac{1}{2}+\sum_{j=1}^{+\infty} \mathcal{C}_{2 j} \tau^{-2 j} \cos (2 j \theta) \quad \text { as } \tau \rightarrow+\infty .
$$

Taking into account the asymptotic behaviour (185) and problem (184) we conclude that the function

$$
w_{1,0,2}\left(\zeta ; x_{1}\right)=\widehat{N}_{1,0,2}^{2}\left(x_{1}\right) Y_{3}(\zeta)
$$

is a solution of (182). Rewriting the asymptotics of $w_{1,0,2}$ as $\tau \rightarrow+\infty$ in terms of $\xi$, we obtain

$$
\varepsilon a^{2} w_{1,0,2}=\varepsilon \widehat{N}_{1,0,2}^{2}\left(\rho^{2} \cos 2 \theta-\frac{a^{2}}{2}+\sum_{j=1}^{+\infty} a^{2 j+2} \mathcal{C}_{2 j} \rho^{-2 j} \cos 2 j \theta\right)
$$

as $\rho a^{-1} \rightarrow+\infty$.

Rewriting the asymptotics of $w_{1,0,2}$ as $\tau \rightarrow+\infty$ in terms of $\xi$, from the explicit form of the function (42) we obtain

$$
\varepsilon w_{1,0,0}=\varepsilon \alpha_{0}^{1}\left(x_{1}\right)\left(\ln \rho+\ln 2-\ln a+\sum_{j=2}^{\infty} a^{2 j} A_{2 j} \rho^{-2 j} \cos 2 j \theta\right)
$$

as $\rho a^{-1} \rightarrow+\infty$.

Suppose that all the $w_{j, k, 2}$ are solutions of the problems (181). Then, by rewriting the asymptotics of the series (180) as $\tau \rightarrow+\infty$ in terms of $\xi$, taking (187) and (188) into account, and following the method of matched asymptotic expansions, we conclude that an additional term appears in the intermediate expansion (150):

$$
u_{\varepsilon}(x)=\sum_{k=0}^{+\infty} \sum_{j=k}^{+\infty} \varepsilon^{j} \ln ^{k} a v_{j, k, 0}\left(\xi ; x_{1}\right)+\varepsilon a^{2} v_{1,0,2}+\cdots
$$

with the asymptotics at zero

$$
v_{1,0,2}\left(\xi ; x_{1}\right) \sim A_{2} \alpha_{0}^{1}\left(x_{1}\right) \rho^{-2} \cos 2 \theta-\frac{1}{2} \widehat{N}_{1,0,2}^{2} \quad \text { as } \rho \rightarrow 0 .
$$

After rewriting the equation and the boundary conditions in terms of the coordinates $\xi$ (see (20), (21), and (22)) and substituting the 'intermediate' expansion (189) and the series (149) into the problem (2), we collect terms with like powers of $\varepsilon$ and $a$. In view of (190) we obtain the following problem for $v_{1,0,2}$ :

$$
\left\{\begin{array}{l}
\Delta_{\xi} v_{1,0,2}=0 \quad \text { in } \Pi, \\
\frac{\partial v_{1,0,2}}{\partial \xi_{2}}=0 \quad \text { on } \Sigma \\
\frac{\partial v_{1,0,2}}{\partial \xi_{1}}\left(\xi ; \pm \frac{\pi}{2}\right)=0 \quad \text { at } \xi_{1}= \pm \frac{\pi}{2}, \\
v_{1,0,2} \sim A_{2} \alpha_{0}^{1}\left(x_{1}\right) \rho^{-2} \cos 2 \theta-\frac{1}{2} \widehat{N}_{1,0,2}^{2} \quad \text { as } \rho \rightarrow 0 .
\end{array}\right.
$$


We introduce into consideration the auxiliary problem

$$
\left\{\begin{array}{l}
-\Delta_{\xi} X_{3}=0 \quad \text { in } \Pi, \\
\frac{\partial X_{3}}{\partial \xi_{2}}=0 \text { on } \Sigma \\
\frac{\partial X_{3}}{\partial \xi_{1}}=0 \quad \text { at } \xi_{1}= \pm \frac{\pi}{2} \\
X_{3} \sim \rho^{-2} \cos 2 \theta \quad \text { as } \rho \rightarrow 0 .
\end{array}\right.
$$

By (28), giving the definition of the function $X(\xi)$ and the boundary-value problem (27), it follows that the function

$$
X_{3}(\xi)=\frac{\partial^{2} X}{\partial \xi_{2}^{2}}(\xi)
$$

is a solution of (192) with asymptotic behaviour as $\xi_{2} \rightarrow+\infty$,

$$
X_{3}(\xi)=O\left(e^{-2 \xi_{2}}\right)
$$

and the asymptotics of this function at zero (as $\rho \rightarrow 0$ ) is as follows:

$$
X_{3}(\xi)=\rho^{-2} \cos 2 \theta+\breve{B}_{0}+\sum_{j=1}^{+\infty} \breve{B}_{2 j} \rho^{2 j} \cos 2 j \theta,
$$

where $\breve{B}_{2 j}, j=0,1, \ldots$, are constants that can be explicitly calculated. By the boundaryvalue problem (192) for $X_{3}(\xi)$ it follows that the function

$$
v_{1,0,2}\left(\xi ; x_{1}\right)=A_{2} \alpha_{0}^{1}\left(x_{1}\right) X_{3}(\xi)+\mathcal{K}_{1,0,2}\left(x_{1}\right)
$$

is a solution of (191) that is $\pi$-periodic and even in $\xi_{1}$, where

$$
\mathcal{K}_{1,0,2}\left(x_{1}\right)=-\frac{1}{2} \widehat{N}_{1,0,2}^{2}\left(x_{1}\right)-\breve{B}_{0} A_{2} \alpha_{0}^{1}\left(x_{1}\right)
$$

and

$$
\frac{\partial^{2 j+1} \mathcal{K}_{1,0,2}}{\partial x_{1}^{2 j+1}}\left( \pm \frac{\pi}{2}\right)=0, \quad j=0,1, \ldots,
$$

by (47) and (14). It follows from (196) and (194) that the asymptotic expression for the function $v_{1,0,2}$ as $\xi_{2} \rightarrow+\infty$ has the form

$$
v_{1,0,2}\left(\xi ; x_{1}\right)=\mathcal{K}_{1,0,2}\left(x_{1}\right)+O\left(e^{-2 \xi_{2}}\right) .
$$

Note that due to (14) and (197) we have

$$
\frac{\partial v_{1,0,2}}{\partial x_{1}}\left(\xi ; x_{1}\right)=0 \quad \text { at } x_{1}= \pm \frac{\pi}{2}
$$

and so, using (21), (22), (23) and (35), we have

$$
\frac{\partial v_{1,0,2}}{\partial \nu}\left(\frac{x}{\varepsilon} ; x_{1}\right)=0 \quad \text { on } \Gamma_{2} \cup \Gamma_{3} .
$$

The more precise asymptotics of the series (189) as $\xi_{2} \rightarrow+\infty$, in view of the asymptotic behaviour (198), makes it necessary for us to introduce new terms in the outer expansion. We seek an outer expansion in the form

$$
u_{\varepsilon}(x)=\sum_{k=0}^{+\infty} \sum_{j=k}^{+\infty} \varepsilon^{j} \ln ^{k} a u_{j, k, 0}(x)+\sum_{k=0}^{+\infty} \sum_{j=k}^{+\infty} a^{2} \varepsilon^{j} \ln ^{k} a u_{j, k, 2}(x)+\cdots,
$$

where

$$
u_{1,0,2} \sim \mathcal{K}_{1,0,2} \quad \text { as } x_{2} \rightarrow 0 .
$$


Corresponding to (201), we seek an asymptotic expansion of the eigenvalue $\lambda_{\varepsilon}$ in the form

$$
\lambda_{\varepsilon}=\sum_{k=0}^{+\infty} \sum_{j=k}^{+\infty} \varepsilon^{j} \ln ^{k} a \lambda_{j, k, 0}+\sum_{k=0}^{+\infty} \sum_{j=k}^{+\infty} a^{2} \varepsilon^{j} \ln ^{k} a \lambda_{j, k, 2}+\cdots .
$$

We seek functions $u_{j, k, 2}$ in $C^{\infty}(\bar{\Omega})$; therefore, in particular, the asymptotic behaviour (202) is equivalent to the boundary conditions

$$
u_{1,0,2}\left(x_{1}, 0\right)=\alpha_{1,0,2}^{0} \equiv \mathcal{K}_{1,0,2}, \quad x_{1} \in\left(-\frac{\pi}{2}, \frac{\pi}{2}\right) .
$$

Substituting the expansion (201) of the function $u_{\varepsilon}$ in terms of $x$ and the expansion (203) into the problem and collecting terms with like powers of $a, \varepsilon, \ln a$, we obtain the following problem for $u_{1,0,2}$ :

$$
\left\{\begin{array}{l}
-\Delta u_{1,0,2}=\lambda_{0} u_{1,0,2}+\lambda_{1,0,2} u_{0} \quad \text { for } x \in \Omega, \\
u_{1,0,2}=\alpha_{1,0,2}^{0} \quad \text { for } x \in \Gamma_{4}, \\
\frac{\partial u_{1,0,2}}{\partial \nu}=0 \text { for } x \in \Gamma_{1} \cup \Gamma_{2} \cup \Gamma_{3}
\end{array}\right.
$$

and, correspondingly, from the same considerations as in Remark 3.1 we obtain the following problems for the $u_{j, k, 2}$ :

$$
\left\{\begin{aligned}
&-\Delta u_{j, k, 2}= \sum_{p=0}^{j} \sum_{q=p}^{k+j-p} \lambda_{p, q, 0} u_{j-p, k-q, 2} \\
&+\sum_{p=0}^{j} \sum_{q=p}^{k+j-p} \lambda_{p, q, 2} u_{j-p, k-q, 0} \quad \text { in } \Omega \\
& u_{j, k, 2}=\alpha_{j, k, 2}^{0} \quad \text { for } x \in \Gamma_{4}, \\
& \frac{\partial u_{j, k, 2}}{\partial \nu}=0 \quad \text { for } x \in \Gamma_{1} \cup \Gamma_{2} \cup \Gamma_{3},
\end{aligned}\right.
$$

where $\alpha_{1,0,2}^{0} \equiv \mathcal{K}_{1,0,2}$, while the other $\alpha_{j, k, 2}^{0}\left(x_{1}\right)$ are so far unknown (arbitrary) functions whose odd derivatives vanish at $x_{1}= \pm \pi / 2$.

Next, from the condition that (205) is solvable, we find $\lambda_{1,0,2}$ and determine $u_{1,0,2}$.

In order that problems (205) and (206) be uniquely solvable, we choose all solutions to be orthogonal to $u_{0}$ in $L_{2}(\Omega)$.

We expand the functions $u_{j, k, 2}(x)$ into a Taylor series in terms of $x_{2}$ as $x_{2} \rightarrow 0$. We make the change of variables $\xi_{2}=x_{2} / \varepsilon$ (that is, we rewrite these expansions in the 'intermediate' variables). Then the asymptotics of the series (201) as $\varepsilon \xi_{2} \rightarrow 0$ has the form

$$
u_{\varepsilon}(x)=\sum_{k=0}^{+\infty} \sum_{j=k}^{+\infty} \varepsilon^{j} \ln ^{k} a V_{j, k, 0}\left(\xi_{2} ; x_{1}\right)+\sum_{k=0}^{+\infty} \sum_{j=k}^{+\infty} a^{2} \varepsilon^{j} \ln ^{k} a V_{j, k, 2}\left(\xi_{2} ; x_{1}\right)+\cdots,
$$

where the $V_{j, k, 0}\left(\xi_{2} ; x_{1}\right)$ are already known, while the $V_{j, k, 2}\left(\xi_{2} ; x_{1}\right)$ are polynomials of order $j-k$ in the variable $\xi_{2}$ whose coefficients are functions of $x_{1}$ that are infinitely differentiable on $[-\pi / 2, \pi / 2]$ and whose odd derivatives vanish at $x_{1}= \pm \pi / 2$. We have the representation

$$
V_{j, k, 2}\left(\xi_{2} ; x_{1}\right)=\widetilde{V}_{j, k, 2}\left(\xi_{2} ; x_{1}\right)+\alpha_{j, k, 2}^{0}\left(x_{1}\right), \quad \widetilde{V}_{1,0,2} \equiv 0,
$$


where the $\widetilde{V}_{j, k, 2}$ are independent of the $\alpha_{s, k, 2}^{0}, s \geq j$. By the boundary-value problems (205), (206) and the definition of the $V_{j, k, 2}$ we have the following equalities:

$$
\begin{aligned}
-\Delta_{\xi} V_{j, k, 2}= & 2 \frac{\partial^{2} V_{j-1, k, 2}}{\partial x_{1} \partial \xi_{1}}+\frac{\partial^{2} V_{j-2, k, 2}}{\partial x_{1}^{2}} \\
& +\sum_{p=0}^{j-2} \sum_{q=p}^{k+j-p} \lambda_{p, q, 0} V_{j-2-p, k-q, 2}+\sum_{p=0}^{j-2} \sum_{q=p}^{k+j-p} \lambda_{p, q, 2} V_{j-2-p, k-q, 0}, \\
& \frac{\partial V_{j, k, 2}}{\partial x_{1}}\left(\xi_{2} ; \pm \frac{\pi}{2}\right)=0, \quad \frac{\partial V_{j, k, 2}}{\partial \xi_{1}}\left(\xi_{2} ; x_{1}\right) \equiv 0 .
\end{aligned}
$$

We note as before that the condition

$$
\frac{d^{2 l+1} \alpha_{j, k, 2}^{0}}{d x_{1}^{2 l+1}}\left( \pm \frac{\pi}{2}\right)=0, \quad l=0,1, \ldots, \quad j=2,3, \ldots, \quad k=0,1, \ldots,
$$

is important and we shall check its validity.

By taking (207) and (201) into account and following the method of matched asymptotic expansions, we see that the intermediate expansion must have the following structure:

$$
u_{\varepsilon}(x)=\sum_{k=0}^{+\infty} \sum_{j=k}^{+\infty} \varepsilon^{j} \ln ^{k} a v_{j, k, 0}\left(\xi ; x_{1}\right)+\sum_{k=0}^{+\infty} \sum_{j=k}^{+\infty} a^{2} \varepsilon^{j} \ln ^{k} a v_{j, k, 2}\left(\xi ; x_{1}\right)+\cdots,
$$

where

$$
v_{q, p, 2}\left(\xi ; x_{1}\right)=V_{q, p, 2}\left(\xi_{2} ; x_{1}\right)+o(1) \quad \text { as } \quad \xi_{2} \rightarrow+\infty .
$$

We substitute the series (211) and the expansion (203) into (2) (first having rewritten it in terms of $\xi$ ) and collect terms with like powers of $a, \varepsilon, \ln a$ in the equation and in the boundary conditions; using (212) we conclude that the problems for the $v_{j, k, 2}$ have the form

$$
\left\{\begin{aligned}
&-\Delta_{\xi} v_{j, k, 2}= 2 \frac{\partial^{2} v_{j-1, k, 2}}{\partial x_{1} \partial \xi_{1}}+\frac{\partial^{2} v_{j-2, k, 2}}{\partial x_{1}^{2}} \\
&+\sum_{p=0}^{j-2} \sum_{q=p}^{k+j-p} \lambda_{p, q, 0} v_{j-2-p, k-q, 2} \\
&+\sum_{p=0}^{j-2} \sum_{q=p}^{k+j-p} \lambda_{p, q, 2} v_{j-2-p, k-q, 0} \quad \text { in } \Pi, \\
& \frac{\partial v_{j, k, 2}}{\partial \xi_{2}}=0 \quad \text { on } \Sigma, \\
& \frac{\partial v_{j, k, 2}}{\partial \xi_{1}}\left(\xi ; \pm \frac{\pi}{2}\right)=-\frac{\partial v_{j-1, k, 2}}{\partial x_{1}}\left(\xi ; \pm \frac{\pi}{2}\right) \quad \text { at } \xi_{1}= \pm \frac{\pi}{2} \\
& v_{j, k, 2} \sim V_{j, k, 2} \quad \text { as } \xi_{2} \rightarrow+\infty .
\end{aligned}\right.
$$

The behaviour of the functions $v_{j, k, 2}$ at zero will be determined more precisely later. We return to analysing problem (181). To do this we introduce the following classes of functions.

- In order to define new classes of functions, we introduce the following notation: $\widetilde{B}$ is the half-disc $\left\{\zeta: \tau<1, \zeta_{2} \geq 0\right\}, \mathcal{O}_{\varsigma, \pm}$ are $\varsigma$-neighbourhoods of the points $( \pm 1,0)$. We denote by $B_{\text {even }}^{(2 k, 2 n, q)}$ the set of functions

$$
g_{\text {even }} \in H_{\text {loc }}^{1}\left(\mathbb{R}_{+}^{2}\right) \cap C^{\infty}(\widetilde{B}) \cap H_{\text {loc }}^{2}\left(\mathbb{R}_{+}^{2} \backslash \overline{\left(\mathcal{O}_{\varsigma,+} \cup \mathcal{O}_{\varsigma,-}\right)}\right) \quad \text { for any } \varsigma
$$


that are even in $\zeta_{1}$ and have an asymptotic expansion at infinity of the form

$$
\begin{aligned}
g_{\text {even }}(\zeta)= & \delta_{0}^{q} \sum_{i=1}^{k+1} \eta_{\text {even }}^{(i)} \tau^{2 i-2} \cos 2 i \theta \\
& +\sum_{i=0}^{k} \sum_{j=0}^{i} \widehat{\eta}_{\text {even }}^{(i, j)} \tau^{2 i} \cos 2(i-j) \theta \ln \tau \\
& +\sum_{i=0}^{\infty} \sum_{j=0}^{i} \widetilde{\eta}_{\text {even }}^{(i, j)} \tau^{-2 i+2 j} \cos 2 i \theta \\
& +\sum_{i=1}^{n} \sum_{j=0}^{i} \breve{\eta}_{\text {even }}^{(i, j)} \tau^{2 i-2 j} \cos 2 i \theta, \quad \tau \rightarrow+\infty
\end{aligned}
$$

where $\delta_{0}^{q}$ is the Kronecker delta. We also point out that

$$
\begin{gathered}
B_{\text {even }}^{(2 k, 2 n, 1)} \subset B_{\text {even }}^{(2 k+2 s, 2 n+2 t, 1)}, \quad B_{\text {even }}^{(2 k, 2 n, 0)} \subset B_{\text {even }}^{(2 k+2 s, 2 n+2 t, 0)}, \\
B_{\text {even }}^{(2 k, 2 n, 1)} \subset B_{\text {even }}^{(2 k+2 s, 2 n+2 t, 0)}, \quad s, t=0,1, \ldots
\end{gathered}
$$

We set

$$
\begin{aligned}
\mathcal{B}_{\text {even }}^{(2 k, 2 n, q)}=\left\{V\left(\zeta ; x_{1}\right):\right. & V\left(\zeta ; x_{1}\right)=\sum_{j=1}^{J} \sigma_{j}\left(x_{1}\right) Y_{j}(\zeta), Y_{j} \in B_{\text {even }}^{(2 k, 2 n, q)}, \\
& \left.\sigma_{j} \in C^{\infty}\left[-\frac{\pi}{2}, \frac{\pi}{2}\right],\left(\sigma_{j}\right)^{(2 n+1)}\left( \pm \frac{\pi}{2}\right)=0, n=0,1, \ldots\right\},
\end{aligned}
$$

where $J$ is arbitrary (not fixed, but finite). We point out that

$$
\frac{\partial V}{\partial x_{1}}(\zeta ; \pm \pi / 2)=0 \quad \text { for all } V \in \mathcal{B}_{\text {even }}^{(2 k, 2 n, q)}
$$

- We denote by $B_{\text {odd }}^{(2 k-1,2 n-1, q)}$ the set of functions

$$
g_{\text {odd }} \in H_{\text {loc }}^{1}\left(\mathbb{R}_{+}^{2}\right) \cap C^{\infty}(\widetilde{B}) \cap H_{\text {loc }}^{2}\left(\mathbb{R}_{+}^{2} \backslash \overline{\left(\mathcal{O}_{\varsigma,+} \cup \mathcal{O}_{\varsigma,-}\right)}\right) \quad \text { for any } \varsigma
$$

that are odd in $\zeta_{1}$ and have asymptotic behaviour at infinity of the form

$$
\begin{aligned}
f_{\text {odd }}(\zeta)= & \delta_{0}^{q} \sum_{i=0}^{k} \eta_{\text {odd }}^{(i)} \tau^{2 i-1} \cos (2 i+1) \theta \\
& +\sum_{i=0}^{k-1} \sum_{j=0}^{i} \widehat{\eta}_{\text {odd }}^{(i, j)} \tau^{2 i+1} \cos (2 i-2 j+1) \theta \ln \tau \\
& +\sum_{i=0}^{\infty} \sum_{j=0}^{i} \widetilde{\eta}_{\text {odd }}^{(i, j)} \tau^{-2 i+2 j-1} \cos (2 i+1) \theta \\
& +\sum_{i=0}^{n-1} \sum_{j=0}^{i} \breve{\eta}_{\text {odd }}^{(i, j)} \tau^{2 i-2 j+1} \cos (2 i+1) \theta, \quad \tau \rightarrow+\infty
\end{aligned}
$$

where $\delta_{0}^{q}$ is the Kronecker delta. We note immediately that the symbol $B_{\text {odd }}^{(2 k-1,2 n-1, q)}$ for $k=0$ denotes the class of functions whose asymptotic expansion as $\tau \rightarrow+\infty$ has no terms with $\ln \tau$; correspondingly, for $n=0$, it denotes the class of functions whose asymptotic expansion as $\tau \rightarrow+\infty$ has no terms with negative powers of $\tau$. 
We observe that

$$
\begin{aligned}
& B_{\text {odd }}^{(2 k-1,2 n-1,1)} \subset B_{\text {odd }}^{(2 k+2 s-1,2 n+2 t-1,1)}, \quad B_{\text {odd }}^{(2 k-1,2 n-1,0)} \\
& B_{\text {odd }}^{(2 k-1,2 n-1,1)} \subset B_{\text {odd }}^{(2 k+2 s-1,2 n+2 t-1,0)},
\end{aligned}
$$

and we denote

$$
\begin{aligned}
\mathcal{B}_{\text {odd }}^{(2 k-1,2 n-1, q)}=\left\{V\left(\zeta ; x_{1}\right):\right. & V\left(\zeta ; x_{1}\right)=\sum_{j=1}^{J} \sigma_{j}\left(x_{1}\right) Y_{j}(\zeta), Y_{j} \in B_{\text {odd }}^{(2 k-1,2 n-1, q)}, \\
& \left.\sigma_{j} \in C^{\infty}\left[-\frac{\pi}{2}, \frac{\pi}{2}\right],\left(\sigma_{j}\right)^{(2 n)}\left( \pm \frac{\pi}{2}\right)=0, n=0,1, \ldots\right\},
\end{aligned}
$$

where $J$ is arbitrary (not fixed, but finite).

It follows immediately from the definition of the classes that the following hold.

1) If $V \in \mathcal{B}_{\text {odd }}^{(2 k-1,2 n-1,1)}$, then

$$
\frac{\partial^{2} V}{\partial \zeta_{1} \partial x_{1}} \in \mathcal{B}_{\text {even }}^{(2 k-2,2 n-2,0)} \quad \text { and } \quad \frac{\partial^{2} V}{\partial x_{1}^{2}} \in \mathcal{B}_{\text {odd }}^{(2 k-1,2 n-1,1)} .
$$

2) If $V \in \mathcal{B}_{\text {even }}^{(2 k, 2 n, 1)}$, then

$$
\frac{\partial^{2} V}{\partial \zeta_{1} \partial x_{1}} \in \mathcal{B}_{\text {odd }}^{(2 k-1,2 n-1,0)} \text { and } \quad \frac{\partial^{2} V}{\partial x_{1}^{2}} \in \mathcal{B}_{\text {even }}^{(2 k, 2 n, 1)} .
$$

We introduce the following class of functions:

$$
\begin{aligned}
\mathbb{B}^{(2 k+1,2 n+1, q)}=\left\{V\left(\zeta ; x_{1}\right):\right. & V\left(\zeta ; x_{1}\right)=\widehat{Z}\left(\zeta ; x_{1}\right)+\check{Z}\left(\zeta ; x_{1}\right), \\
& \left.\widehat{Z} \in \mathcal{B}_{\text {odd }}^{(2 k+1,2 n+1, q)}, \check{Z} \in \mathcal{B}_{\text {even }}^{(2 k, 2 n, q)}\right\}, \\
\mathbb{B}^{(2 k, 2 n, q)}=\left\{V\left(\zeta ; x_{1}\right):\right. & V\left(\zeta ; x_{1}\right)=\widehat{Z}\left(\zeta ; x_{1}\right)+\check{Z}\left(\zeta ; x_{1}\right), \\
& \left.\widehat{Z} \in \mathcal{B}_{\text {odd }}^{(2 k-1,2 n-1, q)}, \check{Z} \in \mathcal{B}_{\text {even }}^{(2 k, 2 n, q)}\right\},
\end{aligned}
$$

where $J$ is arbitrary (finite, but not fixed).

We point out the following properties of these classes:

$$
\begin{gathered}
\mathbb{B}^{(k, n, 1)} \subset \mathbb{B}^{(k+2 s, n+2 t, 1)}, \quad \mathbb{B}^{(k, n, 0)} \subset \mathbb{B}^{(k+2 s, n+2 t, 0)}, \\
\mathbb{B}^{(k, n, 1)} \subset \mathbb{B}^{(k+2 s, n+2 t, 0)}, \quad s, t=0,1, \ldots .
\end{gathered}
$$

- We denote by $H_{\text {odd }}^{(2 k-1)}, k=1, \ldots$, the set of polynomials in $\tau$ of the form

$$
\sum_{i=0}^{k-1} \sum_{j=0}^{i} \varkappa_{\text {odd }}^{(i, j)}\left(x_{1}\right) \tau^{2 i+1} \cos (2 i-2 j+1) \theta,
$$

with infinitely differentiable coefficients on the segment $[-\pi / 2, \pi / 2]$, whose even derivatives with respect to $x_{1}$ vanish at $x_{1}= \pm \pi / 2$.

We denote by $H_{\text {even }}^{(1,2 k)}, k=1, \ldots$, the set of polynomials in $\tau$ of the form

$$
\sum_{i=1}^{k} \sum_{j=0}^{i} \varkappa_{\mathrm{even}}^{(i, j)}\left(x_{1}\right) \tau^{2 i} \cos 2(i-j) \theta
$$

with infinitely differentiable coefficients on the segment $[-\pi / 2, \pi / 2]$, whose odd derivatives with respect to $x_{1}$ vanish at $x_{1}= \pm \pi / 2$. 
We denote by $H_{\text {even }}^{(0,2 k)}, k=0,1, \ldots$, the set of polynomials in $\tau$ of the form

$$
\sum_{i=0}^{k} \sum_{j=0}^{i} \varkappa_{\text {even }}^{(i, j)}\left(x_{1}\right) \tau^{2 i} \cos 2(i-j) \theta
$$

with infinitely differentiable coefficients on the segment $[-\pi / 2, \pi / 2]$, whose odd derivatives with respect to $x_{1}$ vanish at $x_{1}= \pm \pi / 2$.

We also set

$$
\begin{gathered}
F^{(1, k)}=\left\{V\left(\zeta ; x_{1}\right): V\left(\zeta ; x_{1}\right)=\sum_{j=1}^{k} \ln \tau h_{j}\left(\zeta ; x_{1}\right)+\sum_{j=1}^{k} \widetilde{h}_{j}\left(\zeta ; x_{1}\right),\right. \\
\left.h_{2 j-1} \in H_{\text {odd }}^{(2 j-1)}, \widetilde{h}_{2 j-1} \in H_{\text {odd }}^{(2 j-1)}, h_{2 j} \in H_{\text {even }}^{(1,2 j)}, \widetilde{h}_{2 j} \in H_{\text {even }}^{(1,2 j)}\right\}, \\
F^{(0, k)}=\left\{\begin{aligned}
& k\left(\zeta ; x_{1}\right): V\left(\zeta ; x_{1}\right)=\sum_{j=0}^{k} \ln \tau h_{j}\left(\zeta ; x_{1}\right)+\sum_{j=0}^{k} \widetilde{h}_{j}\left(\zeta ; x_{1}\right), \\
&\left.h_{2 j+1} \in H_{\text {odd }}^{(2 j+1)}, \widetilde{h}_{2 j+1} \in H_{\text {odd }}^{(2 j+1)}, h_{2 j} \in H_{\text {even }}^{(0,2 j)}, \widetilde{h}_{2 j} \in H_{\text {even }}^{(0,2 j)}\right\} .
\end{aligned}\right.
\end{gathered}
$$

We observe that the definition of the class $F^{(q, k)}$ implies that $\widetilde{W}_{j, k, 2} \in F^{(1,2)}$.

Theorem 3.6. Let $F \in \mathbb{B}^{(k, n, 0)}$ and suppose that a function $Q \in F^{(1, n+2)}$ satisfies the equality $F-\Delta Q=O\left(\tau^{-2}\right)$ as $\tau \rightarrow+\infty$. Then there exists a solution $W \in \mathbb{B}^{(k+2, n+2,1)}$ of the boundary-value problem

$$
\left\{\begin{array}{l}
\Delta_{\zeta} W=F \quad \text { in } \mathbb{R}_{+}^{2}, \\
\frac{\partial W}{\partial \zeta_{2}}=0 \quad \text { on } \Gamma, \\
W=0 \quad \text { on } \gamma, \\
W \sim Q \quad \text { as } \tau \rightarrow+\infty
\end{array}\right.
$$

Theorem 3.6 will be proved in Appendix 2 (\$4).

By Theorem 3.6 there exists a solution $\widehat{w}_{j, k, 2} \in \mathbb{B}^{j-k, 2,0}$ of problem (181) such that

$$
\widehat{w}_{j, k, 2}=\widehat{W}_{j, k, 2}+O\left(\tau^{-1}\right) \quad \text { as } \quad \tau \rightarrow+\infty,
$$

where

$$
\widehat{W}_{j, k, 2} \in F^{(0,2)}, \quad \widehat{W}_{j, k, 2}-\widetilde{W}_{j, k, 2}=O(1) \quad \text { as } \quad \tau \rightarrow+\infty .
$$

Consider a function of the form

$$
w_{j, k, 2}=\widehat{w}_{j, k, 2}+a_{j, k, 2} Y,
$$

where the function $Y$ was defined in (42), while the coefficients $a_{j, k, 2}$ so far remain arbitrary, but satisfy the property

$$
\frac{d^{2 j+1} a_{2,0,2}}{d x_{1}^{2 j+1}}\left( \pm \frac{\pi}{2}\right)=0, \quad j=0, \ldots .
$$

The function (220) is a solution of problem (181) such that

$$
w_{j, k, 2}=\widehat{W}_{j, k, 2}+a_{j, k, 2} \ln \tau+a_{j, k, 2} \ln 2+O\left(\tau^{-1}\right) \quad \text { as } \tau \rightarrow+\infty .
$$

- We denote by $\mathcal{H}_{\text {odd }}^{2 k-1}\left(\xi ; x_{1}\right)$ the set of polynomials of the form

$$
\sum_{i=0}^{k-1} \sum_{j=0}^{i} \varkappa_{\text {odd }}^{(i, j)}\left(x_{1}\right) \rho^{-2 i-1} \cos (2 i+2 j+1) \theta
$$


with infinitely differentiable coefficients on the segment $[-\pi / 2, \pi / 2]$, whose even derivatives with respect to $x_{1}$ vanish at $x_{1}= \pm \pi / 2$.

We denote by $\mathcal{H}_{\mathrm{even}}^{2 k}\left(\xi ; x_{1}\right)$ the set of polynomials of the form

$$
\sum_{i=0}^{k} \sum_{j=0}^{i} \varkappa_{\text {even }}^{(i, j)}\left(x_{1}\right) \rho^{-2 i} \cos 2(i+j) \theta,
$$

with infinitely differentiable coefficients on the segment $[-\pi / 2, \pi / 2]$, whose odd derivatives with respect to $x_{1}$ vanish at $x_{1}= \pm \pi / 2$.

We also denote

$$
\begin{aligned}
\mathcal{F}^{(k)}=\left\{V\left(\xi ; x_{1}\right):\right. & V\left(\xi ; x_{1}\right)=h_{0}\left(x_{1}\right) \ln \rho+\sum_{j=1}^{k} h_{j}\left(\xi ; x_{1}\right), \\
& \left.h_{2 j+1} \in \mathcal{H}_{\text {odd }}^{2 j+1}, h_{2 j} \in \mathcal{H}_{\text {even }}^{2 j}, j=0, \ldots,\left[\frac{k}{2}\right]\right\} .
\end{aligned}
$$

We rewrite the asymptotics of the series (180) as $\tau \rightarrow+\infty$ in terms of the variables $\xi$, taking (64), (171) and (169) into account:

$$
\begin{aligned}
u_{\varepsilon}= & \sum_{k=0}^{+\infty} \sum_{j=k}^{+\infty} \varepsilon^{j} \ln ^{k} a \mathcal{V}_{j, k, 0}\left(\xi ; x_{1}\right)+\sum_{k=0}^{+\infty} \sum_{j=k}^{+\infty} a^{2} \varepsilon^{j} \ln ^{k} a \mathcal{V}_{j, k, 2}\left(\xi ; x_{1}\right) \\
& +O\left(a^{3} \rho^{-3}\right) \quad \text { as } \quad \rho a^{-1} \rightarrow+\infty, \\
\mathcal{V}_{j, k, 0}= & \mathcal{M}_{j, k}\left(x_{1}\right)(\ln \tau+\ln 2), \quad j \geq k, \\
\mathcal{V}_{j, k, 2}= & \widetilde{\mathcal{V}}_{j, k, 2}+a_{j, k, 2} \ln \rho+a_{j, k, 2} \ln 2,
\end{aligned}
$$

where $\widetilde{\mathcal{V}}_{j, k, 2} \in \mathcal{F}^{(2)}$ is independent of $a_{p, q, 2}, p \geq j, q \geq k$.

Taking the reexpression of the asymptotics in (223) into account, we conclude that solutions of the problem (213) must have asymptotic behaviour

$$
\begin{gathered}
v_{j, k, 2}\left(\xi ; x_{1}\right)=\widetilde{\mathcal{V}}_{j, k, 2}\left(\xi ; x_{1}\right)+a_{j, k, 2}\left(x_{1}\right) \ln \rho+a_{j, k, 2}\left(x_{1}\right) \ln 2 \\
+O(\rho \ln \rho) \quad \text { as } \rho \rightarrow 0 .
\end{gathered}
$$

To analyse problems (213), (224), we introduce the following classes of functions.

- We denote by $A_{\text {even }}^{(2 k, 2 n, q)}$ the set of functions $f_{\text {even }} \in C^{\infty}\left(\overline{\mathbb{R}_{+}^{2}} \backslash \bigcup_{k \in \mathbb{Z}}\{(k \pi, 0)\}\right)$ that are $\pi$-periodic and even in $\xi_{1}$, have an asymptotic expansion as $\rho \rightarrow 0$ at zero of the form

$$
\begin{aligned}
f_{\text {even }}(\xi)= & \delta_{0}^{q} \sum_{i=1}^{k+1} \beta_{\text {even }}^{(i)} \rho^{2 i-2} \cos 2 i \theta+\sum_{i=0}^{k} \sum_{j=0}^{i} \widehat{\beta}_{\text {even }}^{(i, j)} \rho^{2 i} \cos 2(i-j) \theta \ln \rho \\
& +\sum_{i=0}^{\infty} \sum_{j=0}^{i} \widetilde{\beta}_{\text {even }}^{(i, j)} \rho^{2 i} \cos 2(i-j) \theta+\sum_{i=1}^{n} \sum_{j=0}^{i} \breve{\beta}_{\text {even }}^{(i, j)} \rho^{-2 i+2 j} \cos 2 i \theta, \\
\rho \rightarrow 0, &
\end{aligned}
$$

where $\delta_{0}^{q}$ is the Kronecker delta, and have the property

$$
e^{\varpi \xi_{2}} \chi(\rho) f_{\text {even }} \in H^{1}(\Pi), \quad 0<\varpi<2 .
$$

We also point out that

$$
\begin{gathered}
A_{\text {even }}^{(2 k, 2 n, 1)} \subset A_{\text {even }}^{(2 k+2 s, 2 n+2 t, 1)}, \quad A_{\text {even }}^{(2 k, 2 n, 0)} \subset A_{\text {even }}^{(2 k+2 s, 2 n+2 t, 0),}, \\
A_{\text {even }}^{(2 k, 2 n, 1)} \subset A_{\text {even }}^{(2 k+2 s, 2 n+2 t, 0)}, \quad s, t=0,1, \ldots .
\end{gathered}
$$


We set

$$
\begin{aligned}
\mathcal{A}_{\text {even }}^{(2 k, 2 n, q)}=\left\{V\left(\xi ; x_{1}\right):\right. & V\left(\xi ; x_{1}\right)=\sum_{j=1}^{J} \sigma_{j}\left(x_{1}\right) Y_{j}(\xi), Y_{j} \in A_{\text {even }}^{(2 k, 2 n, q)}, \\
& \left.\sigma_{j} \in C^{\infty}\left[-\frac{\pi}{2}, \frac{\pi}{2}\right],\left(\sigma_{j}\right)^{(2 n+1)}\left( \pm \frac{\pi}{2}\right)=0, n=0,1, \ldots\right\},
\end{aligned}
$$

where $J$ is arbitrary (not fixed, but finite). We note that

$$
\frac{\partial V}{\partial x_{1}}\left(\xi ; \pm \frac{\pi}{2}\right)=0 \quad \text { for all } V \in \mathcal{A}_{\mathrm{even}}^{(2 k, 2 n, q)} .
$$

- We denote by $A_{\text {odd }}^{(2 k-1,2 n-1, q)}$ the set of functions

$$
f_{\text {odd }} \in C^{\infty}\left(\overline{\mathbb{R}_{+}^{2}} \backslash \bigcup_{k \in \mathbb{Z}}\{(k \pi, 0)\}\right)
$$

that are $\pi$-periodic and odd in $\xi_{1}$, have an asymptotic expansion as $\rho \rightarrow 0$ at zero of the form

$$
\begin{aligned}
f_{\text {odd }}(\xi)= & \delta_{0}^{q} \sum_{i=0}^{k} \beta_{\text {odd }}^{(i)} \rho^{2 i-1} \cos (2 i+1) \theta+\sum_{i=0}^{k-1} \sum_{j=0}^{i} \widehat{\beta}_{\text {odd }}^{(i, j)} \rho^{2 i+1} \cos (2 i-2 j+1) \theta \ln \rho \\
& +\sum_{i=0}^{\infty} \sum_{j=0}^{i} \widetilde{\beta}_{\text {odd }}^{(i, j)} \rho^{2 i+1} \cos (2 i-2 j+1) \theta+\sum_{i=0}^{n-1} \sum_{j=0}^{i} \breve{\beta}_{\text {odd }}^{(i, j)} \rho^{-2 i+2 j-1} \cos (2 i+1) \theta,
\end{aligned}
$$

where $\delta_{0}^{q}$ is the Kronecker delta, and have the property

$$
e^{\varpi \xi_{2}} \chi(\rho) f_{\text {odd }} \in H^{1}(\Pi), \quad 0<\varpi<2 .
$$

We point out at once that the symbol $A_{\text {odd }}^{(2 k-1,2 n-1, q)}$ for $k=0$ denotes the class of functions whose asymptotic expansion as $\rho \rightarrow 0$ has no terms involving $\ln \rho$; correspondingly, for $n=0$ it denotes the class of functions whose asymptotics as $\rho \rightarrow 0$ has no terms with negative powers of $\rho$.

We observe that

$$
\begin{gathered}
A_{\text {odd }}^{(2 k-1,2 n-1,1)} \subset A_{\text {odd }}^{(2 k+2 s-1,2 n+2 t-1,1)}, \quad A_{\text {odd }}^{(2 k-1,2 n-1,0)} \\
A_{\text {odd }}^{(2 k-1,2 n-1,1)} \subset A_{\text {odd }}^{(2 k+2 s-1,2 n+2 t-1,0)}, \quad s=0,1, \ldots .
\end{gathered}
$$

We also denote

$$
\begin{aligned}
\mathcal{A}_{\mathrm{odd}}^{(2 k-1,2 n-1, q)}=\left\{V\left(\xi ; x_{1}\right):\right. & V\left(\xi ; x_{1}\right)=\sum_{j=1}^{J} \sigma_{j}\left(x_{1}\right) Y_{j}(\xi), Y_{j} \in A_{\mathrm{odd}}^{(2 k-1,2 n-1, q)}, \\
& \left.\sigma_{j} \in C^{\infty}\left[-\frac{\pi}{2}, \frac{\pi}{2}\right],\left(\sigma_{j}\right)^{(2 n)}\left( \pm \frac{\pi}{2}\right)=0, n=0,1, \ldots\right\},
\end{aligned}
$$

where $J$ is arbitrary (not fixed and finite).

It follows immediately from the definition that the following hold.

1) If $V \in \mathcal{A}_{\text {odd }}^{(2 k-1,2 n-1,1)}$, then

$$
\frac{\partial^{2} V}{\partial \xi_{1} \partial x_{1}} \in \mathcal{A}_{\text {even }}^{(2 k-2,2 n-2,0)} \quad \text { and } \quad \frac{\partial^{2} V}{\partial x_{1}^{2}} \in \mathcal{A}_{\text {odd }}^{(2 k-1,2 n-1,1)} \text {. }
$$

2) If $V \in \mathcal{A}_{\text {even }}^{(2 k, 2 n, 1)}$, then

$$
\frac{\partial^{2} V}{\partial \xi_{1} \partial x_{1}} \in \mathcal{A}_{\text {odd }}^{(2 k-1,2 n-1,0)} \quad \text { and } \quad \frac{\partial^{2} V}{\partial x_{1}^{2}} \in \mathcal{A}_{\text {even }}^{(2 k, 2 n, 1)} .
$$


We introduce the following class of functions:

$$
\begin{aligned}
& \mathbb{A}^{(2 k+1,2 n+1, q)}=\left\{V\left(\xi ; x_{1}\right): V\left(\xi ; x_{1}\right)=\widehat{Z}\left(\xi ; x_{1}\right)+\check{Z}\left(\xi ; x_{1}\right),\right. \\
& \left.\widehat{Z} \in \mathcal{A}_{\text {odd }}^{(2 k+1,2 n+1, q)}, \quad \check{Z} \in \mathcal{A}_{\text {even }}^{(2 k, 2 n, q)}\right\}, \\
& \mathbb{A}^{(2 k, 2 n, q)}=\left\{V\left(\xi ; x_{1}\right): V\left(\xi ; x_{1}\right)=\widehat{Z}\left(\xi ; x_{1}\right)+\check{Z}\left(\xi ; x_{1}\right),\right. \\
& \left.\widehat{Z} \in \mathcal{A}_{\text {odd }}^{(2 k-1,2 n-1, q)}, \quad \check{Z} \in \mathcal{A}_{\text {even }}^{(2 k, 2 n, q)}\right\},
\end{aligned}
$$

where $J$ is arbitrary (finite but not fixed).

These classes have the following properties:

$$
\begin{gathered}
\mathbb{A}^{(k, n, 1)} \subset \mathbb{A}^{(k+2 s, n+2 t, 1)}, \quad \mathbb{A}^{(k, n, 0)} \subset \mathbb{A}^{(k+2 s, n+2 t, 0)}, \\
\mathbb{A}^{(k, n, 1)} \subset \mathbb{A}^{(k+2 s, n+2 t, 0)}, \quad s, t=0,1, \ldots .
\end{gathered}
$$

The following theorem is proved similarly to Theorem 3.1 .

Theorem 3.7. a) Let $F$ be represented in the form $F=\mathcal{P}_{t}+f$, where $f \in \mathbb{A}^{(k, n, 0)}$ and $\mathcal{P}_{t+2}$ is a polynomial such that $-\mathcal{P}_{t+2}^{\prime \prime}=\mathcal{P}_{t}$. Suppose that a function $\mathcal{Q} \in \mathcal{F}^{(n+2)}$ satisfies the equality $F-\Delta \mathcal{Q}=O\left(\rho^{-1}\right)$ as $\rho \rightarrow 0$. Then there exists a solution of the boundary-value problem

$$
\left\{\begin{array}{l}
\Delta_{\xi} W=F \quad \text { in } \Pi, \\
\frac{\partial W}{\partial \xi_{2}}=0 \quad \text { on } \Sigma, \\
\frac{\partial W}{\partial \xi_{1}}=0 \quad \text { at } \xi_{1}= \pm \frac{\pi}{2}, x_{1}= \pm \frac{\pi}{2}, \\
W=\mathcal{Q}+a \ln \rho+\widetilde{a}+O\left(\rho^{-1}\right) \quad \text { as } \rho \rightarrow 0
\end{array}\right.
$$

that can be represented in the form $W=\mathcal{P}_{t+2}+W_{1}$, where $W_{1} \in \mathbb{A}^{(k+2, n+2,1)}$.

b) The function $W$ satisfies the equalities

$$
\frac{\partial W}{\partial x_{1}}\left(\xi ; \pm \frac{\pi}{2}\right)=0 \quad \text { at } \xi_{1}= \pm \frac{\pi}{2}, \quad \frac{\partial W}{\partial x_{1}}\left(\frac{x}{\varepsilon} ; x_{1}\right)=0 \quad \text { at } x_{1}= \pm \frac{\pi}{2} .
$$

The definition of the classes ensures that the following statement is true.

Lemma 3.5. Suppose that the $v_{j, k, 2}=V_{j, k, 2}+\widetilde{v}_{j, k, 2}$, where $\widetilde{v}_{j, k, 2} \in \mathbb{A}^{(j-k, 2,1)}$, are solutions of the problems

$$
\left\{\begin{aligned}
&-\Delta_{\xi} v_{j, k, 2}= 2 \frac{\partial^{2} v_{j-1, k, 2}}{\partial x_{1} \partial \xi_{1}}+\frac{\partial^{2} v_{j-2, k, 2}}{\partial x_{1}^{2}}+\sum_{p=0}^{j-2} \sum_{q=p}^{k+j-p} \lambda_{p, q, 0} v_{j-2-p, k-q, 2} \\
&+\sum_{p=0}^{j-2} \sum_{q=p}^{k+j-p} \lambda_{p, q, 2} v_{j-2-p, k-q, 0} \quad \text { in } \Pi, \\
& \frac{\partial v_{j, k, 2}}{\partial \xi_{2}}=0 \quad \text { on } \Sigma, \\
& \frac{\partial v_{j, k, 2}}{\partial \xi_{1}}\left(\xi ; \pm \frac{\pi}{2}\right)=0 \quad \text { at } \xi_{1}= \pm \frac{\pi}{2} \\
& v_{j, k, 2}=V_{j, k, 2}+o(1) \quad \text { as } \xi_{2} \rightarrow+\infty
\end{aligned}\right.
$$

Then the $v_{j, k, 2}$ are solutions of problems (213). 
Remark 3.6. As in the preceding sections, we will seek solutions of the problems (213), (224) by turns. Having found the term $v_{1,0,2}=V_{1,0,2}+\widetilde{v}_{1,0,2}$ with $\widetilde{v}_{1,0,2} \in \mathbb{A}^{(1,2,1)}$, we then construct the terms $v_{p, 0,2}=V_{p, 0,2}+\widetilde{v}_{p, 0,2}$ with $\widetilde{v}_{p, 0,2} \in \mathbb{A}^{(p, 2,1)}$ consecutively, for $p=2,3, \ldots$ At the second step we construct $v_{p, 1,2}=V_{p, 1,2}+\widetilde{v}_{p, 1,2}$ with $\widetilde{v}_{p, 1,2} \in \mathbb{A}^{(p-1,2,1)}$ for $p=1,2, \ldots$, and so on. Therefore for fixed indices, in problem (213), (224), on the right-hand side of the equation, which is denoted by $F_{j, k, 2}$, there will be functions that by now are known, and consequently we shall have the equality

$$
-\Delta \mathcal{V}_{j, k, 2}=F_{j, k, 2}+O\left(\rho^{-1}\right) \quad \text { as } \quad \rho \rightarrow 0 .
$$

By Theorem 3.7 there exists a solution of the problem (213), (224) that can be represented in the form $v_{j, k, 2}\left(\xi ; x_{1}\right)=V_{j, k, 2}\left(\xi ; x_{1}\right)+\widetilde{v}_{j, k, 2}\left(\xi ; x_{1}\right)$, where $\widetilde{v}_{j, k, 2} \in \mathbb{A}^{(j-k, 2,1)}$, and has the following asymptotics at zero:

$$
\begin{aligned}
v_{j, k, 2}\left(\xi ; x_{1}\right)= & \widetilde{\mathcal{V}}_{j, k, 2}\left(\xi ; x_{1}\right)+\widehat{\mathcal{M}}_{j, k, 2}\left(x_{1}\right) \ln \rho \\
& +\widetilde{\mathcal{M}}_{j, k, 2}\left(x_{1}\right)+\alpha_{j, k, 2}^{0}\left(x_{1}\right)+O(\rho \ln \rho) \quad \text { as } \quad \rho \rightarrow 0,
\end{aligned}
$$

where $\widehat{\mathcal{M}}_{j, k, 2}\left(x_{1}\right)$ and $\widetilde{\mathcal{M}}_{j, k, 2}\left(x_{1}\right)$ are completely defined functions that are infinitely differentiable on the segment $[-\pi / 2, \pi / 2]$ and whose odd derivatives vanish at $x_{1}= \pm \pi / 2$, while $\alpha_{j, k, 2}^{0}\left(x_{1}\right)$ is arbitrary at present.

On the other hand, the asymptotics must have the form (224). We now show that this can be achieved by choosing the $a_{j, k, 2}$ and $\alpha_{j, k, 2}^{0}$, which so far have been arbitrary. By choosing

$$
a_{j, k, 2}=\widehat{\mathcal{M}}_{j, k, 2}, \quad \alpha_{j, k, 2}=\widehat{\mathcal{M}}_{j, k, 2} \ln 2-\widetilde{\mathcal{M}}_{j, k, 2}
$$

we verify that the function $v_{j, k, 2}$ now has the asymptotics (224); that is, we finally determine the function $v_{j, k, 2}$, and so we finally fix the function $w_{j, k, 2}$. Thus we determine the boundary condition for $u_{j, k, 2}$ in the problem (206); then, from the condition that the problem is solvable, we find $\lambda_{j, k, 2}$ and determine $u_{j, k, 2}$.

Finally, at this step we completely determine the terms $w_{j, k, 2}, v_{j, k, 2}, \lambda_{j, k, 2}$, and $u_{j, k, 2}$.

We conduct the subsequent steps (for $a^{3}, a^{4}, \ldots$ ) along the same lines as we used for the terms for $a^{2}$. As a result we see that the following assertion holds.

Theorem 3.8. There exist series

$$
\begin{gathered}
\lambda_{\varepsilon}=\lambda_{0}+\sum_{j=1}^{\infty} \sum_{l=2}^{\infty} \sum_{k=0}^{j-l+1} \varepsilon^{j} \ln ^{k} a a^{l} \lambda_{j, k, l}+\sum_{j=1}^{\infty} \sum_{k=0}^{j} \varepsilon^{j} \ln ^{k} a \lambda_{j, k, 0}, \\
u_{\varepsilon}(x)=u_{0}(x)+\sum_{j=1}^{\infty} \sum_{l=2}^{\infty} \sum_{k=0}^{j-l+1} \varepsilon^{j} \ln ^{k} a a^{l} u_{j, k, l}(x)+\sum_{j=1}^{\infty} \sum_{k=0}^{j} \varepsilon^{j} \ln ^{k} a u_{j, k, 0}(x), \\
u_{\varepsilon}(x)=\sum_{j=1}^{\infty} \sum_{l=2}^{\infty} \sum_{k=0}^{j-l+1} \varepsilon^{j} \ln ^{k} a a^{l} v_{j, k, l}\left(\xi ; x_{1}\right)+\sum_{j=1}^{\infty} \sum_{k=0}^{j} \varepsilon^{j} \ln ^{k} a v_{j, k, 0}\left(\xi ; x_{1}\right), \\
u_{\varepsilon}(x)=\sum_{j=1}^{\infty} \sum_{l=1}^{\infty} \sum_{k=0}^{j-l} \varepsilon^{j} \ln ^{k} a a^{l} w_{j, k, l}\left(\zeta ; x_{1}\right)+\sum_{j=1}^{\infty} \sum_{k=0}^{j-1} \varepsilon^{j} \ln ^{k} a w_{j, k, 0}\left(\zeta ; x_{1}\right),
\end{gathered}
$$

such that 
a) the pairs $u_{j, k, l} \in C^{\infty}(\bar{\Omega})$ and $\lambda_{j, k, l}$ are solutions of the problems

$$
\left\{\begin{aligned}
-\Delta u_{j, k, l}= & \sum_{s=2}^{l-2} \sum_{p=0}^{j} \sum_{q=p}^{k+j-p} \lambda_{p, q, s} u_{j-p, k-q, l-s} \\
& +\sum_{p=0}^{j} \sum_{q=p}^{k+j-p} \lambda_{p, q, 0} u_{j-p, k-q, l}+\sum_{p=0}^{j} \sum_{q=p}^{k+j-p} \lambda_{p, q, l} u_{j-p, k-q, 0} \quad \text { in } \Omega, \\
\frac{\partial u_{j, k}}{\partial \nu}= & 0 \quad \text { on } \Gamma_{1} \cup \Gamma_{2} \cup \Gamma_{3}, \\
u_{j, k}= & \alpha_{j, k}^{0} \text { on } \Gamma_{4},
\end{aligned}\right.
$$

where $u_{0,0,0}=u_{0}$ and $\lambda_{0,0,0}=\lambda_{0}$;

b) the series (234) has an asymptotic expansion as $x_{2} \rightarrow 0$, rewritten in terms of the variables $\xi$, of the form

$$
\begin{aligned}
u_{\varepsilon}(x)= & \sum_{j=1}^{\infty} \sum_{l=2}^{\infty} \sum_{k=0}^{j-l+1} \varepsilon^{j} \ln ^{k} a a^{l} V_{j, k, l}\left(\xi_{2} ; x_{1}\right) \\
& +\sum_{j=1}^{\infty} \sum_{k=0}^{j} \varepsilon^{j} \ln ^{k} a V_{j, k, 0}\left(\xi_{2} ; x_{1}\right) \quad \text { as } \quad \varepsilon \xi_{2} \rightarrow 0
\end{aligned}
$$

where the $V_{j, k, l}\left(\xi_{2} ; x_{1}\right)$ are polynomials of order $j-k$ in the variable $\xi_{2}$ whose coefficients are functions of $x_{1}$ that are infinitely differentiable on $[-\pi / 2, \pi / 2]$ and whose odd derivatives vanish at $x_{1}= \pm \pi / 2$;

c) the coefficient $\lambda_{1,1,0}$ is determined from formula (122);

d) the functions $v_{j, k, l}$ can be represented as the sums $v_{j, k, l}=V_{j, k, l}+\widetilde{v}_{j, k, l}$, where $\widetilde{v}_{j, k, l} \in \mathbb{A}^{(j-k, l, 1)}$ and $v_{j, j, 0}=\mathcal{M}_{j, j}$, and are solutions of the problem

$$
\left\{\begin{array}{l}
-\Delta_{\xi} v_{j, k, l}=2 \frac{\partial^{2} v_{j-1, k, l}}{\partial x_{1} \partial \xi_{1}}+\frac{\partial^{2} v_{j-2, k, l}}{\partial x_{1}^{2}}+\sum_{s=2}^{l-2} \sum_{p=0}^{j-2} \sum_{q=p}^{k+j-p} \lambda_{p, q, s} v_{j-2-p, k-q, l-s} \\
\quad+\sum_{p=0}^{j-2} \sum_{q=p}^{k+j-p} \lambda_{p, q, 0} v_{j-2-p, k-q, l}+\sum_{p=0}^{j-2} \sum_{q=p}^{k+j-p} \lambda_{p, q, l} v_{j-2-p, k-q, 0} \quad \text { in } \Pi, \\
\frac{\partial v_{j, k, l}}{\partial \xi_{2}}=0 \quad \text { on } \Sigma, \\
\frac{\partial v_{j, k, l}}{\partial \xi_{1}}\left(\xi ; \pm \frac{\pi}{2}\right)=0 \quad \text { at } \xi_{1}= \pm \frac{\pi}{2} ;
\end{array}\right.
$$

furthermore,

$$
\frac{\partial v_{j, k, l}}{\partial x_{1}}\left(\xi ; \pm \frac{\pi}{2}\right) \equiv 0 \quad \text { and } \quad \frac{d^{2 l+1} \mathcal{M}_{j, j}}{d x_{1}^{2 l+1}}\left( \pm \frac{\pi}{2}\right)=0, \quad l=0,1, \ldots
$$

e) the functions $v_{j, k, l}$ have the asymptotic behaviour

$$
v_{j, k, l}=\mathcal{V}_{j, k, l} \quad \text { as } \quad \rho \rightarrow 0,
$$

where $\mathcal{V}_{j, k, l} \in \mathcal{F}^{(l-j+k)}$; 
f) the functions $w_{k, j, l} \in \mathbb{B}^{j-k, l, 1}$ are solutions of the problem

$$
\left\{\begin{aligned}
&-\Delta_{\zeta} w_{j, k, l}= 2 \frac{\partial^{2} w_{j-1, k, l-1}}{\partial x_{1} \partial \zeta_{1}}+\frac{\partial^{2} w_{j-2, k, l-2}}{\partial x_{1}^{2}} \\
&+\sum_{s=0}^{l-2} \sum_{p=0}^{j-2} \sum_{q=p}^{k+j-p} \lambda_{p, q, s} w_{j-2-p, k-q, l-2-s} \quad \text { in } \mathbb{R}_{+}^{2} \\
& \frac{\partial w_{j, k, l}}{\partial \zeta_{2}}=0 \quad \text { on } \Gamma \\
& w_{j, k, l}=0 \quad \text { on } \gamma ;
\end{aligned}\right.
$$

g) the series (236) has an asymptotic expansion as $\tau \rightarrow+\infty$, rewritten in terms of the variables $\xi$, of the form

$$
\begin{aligned}
u_{\varepsilon}(x)= & \sum_{j=1}^{\infty} \sum_{l=2}^{\infty} \sum_{k=0}^{j-l+1} \varepsilon^{j} \ln ^{k} a a^{l} \mathcal{V}_{j, k, l}\left(\xi ; x_{1}\right) \\
& +\sum_{j=1}^{\infty} \sum_{k=0}^{j} \varepsilon^{j} \ln ^{k} a \mathcal{V}_{j, k, 0}\left(\xi ; x_{1}\right) \quad \text { as } \quad \rho a^{-1} \rightarrow+\infty
\end{aligned}
$$

3.4. The construction of the asymptotics. Fourth step. To construct the terms that depend on the concentrated masses, we introduce the following notation. We set $\mu:=\varepsilon^{2-m} a^{2-m}$; then, in the new notation, the density $\rho_{\varepsilon}(x)$ defined in (3) has the form

$$
\rho_{\varepsilon}(x)=\left\{\begin{array}{l}
1 \text { in } \Omega \backslash \bar{B}_{\varepsilon}, \\
1+\mu(a \varepsilon)^{-2}
\end{array} \text { in } B_{\varepsilon} .\right.
$$

We assume the small parameter $\mu$ to be independent. $\tilde{\lambda}$

We denote the series (236) by $\widetilde{w}_{\varepsilon}$, the series (233) by $\widetilde{\lambda}_{\varepsilon}$, the series (234) by $\widetilde{u}_{\varepsilon}$ and the series (235) by $\widetilde{v}_{\varepsilon}$. Substituting $\widetilde{w}_{\varepsilon}$ and $\widetilde{\lambda}_{\varepsilon}$ into the equation in problem (2), we obtain

$$
-\Delta \widetilde{w}_{\varepsilon}=\rho_{\varepsilon} \widetilde{\lambda}_{\varepsilon} \widetilde{w}_{\varepsilon}=\widetilde{\lambda}_{\varepsilon} \widetilde{w}_{\varepsilon}+\mu(a \varepsilon)^{-2} \widetilde{\lambda}_{\varepsilon} \widetilde{w}_{\varepsilon} .
$$

Hence it immediately follows that we need to introduce new terms in the inner expansion. We seek an inner expansion in the form

$$
u_{\varepsilon}=\widetilde{w}_{\varepsilon}+\mu \widehat{w}_{\varepsilon}+\cdots
$$

and, correspondingly,

$$
\lambda_{\varepsilon}=\tilde{\lambda}_{\varepsilon}+\mu \widehat{\lambda}_{\varepsilon}+\cdots .
$$

Substituting the series (245) and (246) into (2) and collecting terms for the corresponding minimal powers of $a, \varepsilon, \ln a$, and $\mu$, we conclude that

$$
\widehat{w}_{\varepsilon}=\varepsilon w_{1,0,0,1}+\cdots
$$

and the problem for the function $w_{1,0,0,1}$ has the form

$$
\left\{\begin{array}{l}
-\Delta_{\zeta} w_{1,0,0,1}=\left\{\begin{array}{l}
0 \text { in } \overline{\mathbb{R}_{+}^{2}} \backslash B, \\
\lambda_{0} w_{1,0,0,0} \text { in } B,
\end{array}\right. \\
w_{1,0,0,1}=0 \text { on } \gamma, \\
\frac{\partial w_{1,0,0,1}}{\partial \zeta_{2}}=0 \quad \text { on } \Gamma,
\end{array}\right.
$$


where $w_{1,0,0,0} \equiv w_{1,0,0}$. We consider the auxiliary problem

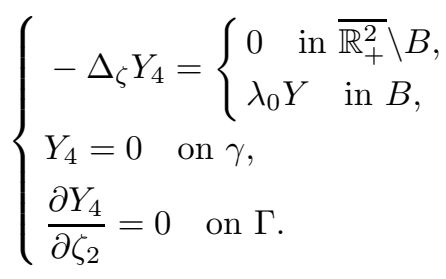

Lemma 3.6. Suppose that $F \in L_{2}\left(\mathbb{R}_{+}^{2}\right) \cap C^{\infty}(\widetilde{B})$ has the asymptotic behaviour

$$
F=O\left(\tau^{-N-1}\right) \quad \text { as } \quad \tau \rightarrow+\infty .
$$

Then there exists a bounded function

$$
W \in H_{\text {loc }}^{1}\left(\mathbb{R}_{+}^{2}\right) \cap C^{\infty}(\widetilde{B}) \cap H_{\text {loc }}^{2}\left(\mathbb{R}_{+}^{2} \backslash \overline{\left(\mathcal{O}_{\varsigma,+} \cup \mathcal{O}_{\varsigma,-}\right)}\right),
$$

for arbitrary $\varsigma$, that is a solution of the boundary-value problem

$$
\left\{\begin{array}{l}
\Delta_{\zeta} W=F \quad \text { in } \mathbb{R}_{+}^{2}, \\
\frac{\partial W}{\partial \zeta_{2}}=0 \quad \text { on } \Gamma \\
W=0 \quad \text { on } \gamma
\end{array}\right.
$$

with the asymptotic expansion

$$
W=\sum_{j=0}^{N-2} \widetilde{c}_{j} \tau^{-j} \cos (j \theta)+O\left(\tau^{-N+1}\right) \quad \text { as } \quad \tau \rightarrow \infty .
$$

If in addition $F$ is even, then $\widetilde{c}_{2 j+1}=0$; if $F$ is odd, then $\widetilde{c}_{2 j}=0$.

Lemma 3.6 will be proved in Appendix 2 (\$4). It follows from Lemma 3.6 and the arbitrariness in the choice of $N$ that there exists a bounded solution of (249) having asymptotics

$$
Y_{4} \sim M_{4}+A_{4,2} \tau^{-2} \cos 2 \theta+\sum_{j=2}^{\infty} A_{4,2 j} \tau^{-2 j} \cos 2 j \theta \quad \text { as } \quad \tau \rightarrow+\infty .
$$

It is easy to compute the constant $M_{4}$. First multiply the equation in problem (249) by the function $Y$ defined in (42) and integrate over the half-disc $B_{R}=\left\{\zeta \in \mathbb{R}_{+}^{2},|\zeta|<R\right\}$. Then apply Green's formula twice and pass to the limit as $R \rightarrow+\infty$ to obtain

$$
\pi M_{4}=\lambda_{0} \int_{B} Y^{2}(\zeta) d \zeta>0
$$

Obviously, the function

$$
w_{1,0,0,1}\left(\zeta, x_{1}\right)=\alpha_{0}^{1}\left(x_{1}\right) Y_{4}(\zeta)
$$

satisfies (248) and has the following asymptotics (as $\tau \rightarrow+\infty)$ :

$$
w_{1,0,0,1} \sim \alpha_{0}^{1}\left(M_{4}+A_{4,2} \tau^{-2} \cos 2 \theta+\sum_{j=2}^{\infty} A_{4,2 j} \tau^{-2 j} \cos 2 j \theta\right) .
$$

The appearance of the function $M_{4} \alpha_{0}^{1}\left(x_{1}\right)$ multiplied by $\varepsilon \mu$ in the inner expansion gives rise to a discrepancy in the intermediate expansion. Rewriting the asymptotics of the series (245) as $\tau \rightarrow+\infty$ in terms of the variables $\xi$ and following the matching method, we see that in the intermediate expansion there must be a term $\varepsilon \mu v_{1,0,0,1}$ with the asymptotics

$$
v_{1,0,0,1}\left(\xi ; x_{1}\right) \sim M_{4} \alpha_{0}^{1}\left(x_{1}\right) \quad \text { as } \quad \rho \rightarrow 0
$$


that is, the intermediate expansion has the form

$$
u_{\varepsilon}(x)=\widetilde{v}_{\varepsilon}+\varepsilon \mu v_{1,0,0,1}+\cdots,
$$

where $\widetilde{v}_{\varepsilon}$ is the series (235). After substituting the series (257) and (246) into the problem (2) we collect like terms for the powers of $\varepsilon, a$, and $\ln a$. In view of (256) we obtain the following problem for $v_{1,0,0,1}$ :

$$
\left\{\begin{array}{l}
\Delta_{\xi} v_{1,0,0,1}=0 \quad \text { in } \Pi, \\
\frac{\partial v_{1,0,0,1}}{\partial \xi_{2}}=0 \quad \text { on } \Sigma, \\
\frac{\partial v_{1,0,0,1}}{\partial \xi_{1}}\left(\xi ; \pm \frac{\pi}{2}\right)=0 \quad \text { at } \xi_{1}= \pm \frac{\pi}{2}, \\
v_{1,0,0,1}\left(\xi ; x_{1}\right) \sim M_{4} \alpha_{0}^{1}\left(x_{1}\right) \quad \text { as } \rho \rightarrow 0 .
\end{array}\right.
$$

Obviously, the function $v_{1,0,0,1} \equiv M_{4} \alpha_{0}^{1}\left(x_{1}\right)$ is a solution of (258) with asymptotics at infinity given by

$$
v_{1,0,0,1}=M_{4} \alpha_{0}^{1} \quad \text { as } \quad \xi_{2} \rightarrow+\infty .
$$

Matching the asymptotic expansions, we introduce the following term into the outer expansion:

$$
u_{\varepsilon}(x)=\widetilde{u}_{\varepsilon}+\varepsilon \mu u_{1,0,0,1}+\cdots,
$$

where $\widetilde{u}_{\varepsilon}$ is the series (234), while $u_{1,0,0,1} \sim M_{4} \alpha_{0}^{1}\left(x_{1}\right)$ as $x_{2} \rightarrow 0$. We seek the function $u_{1,0,0,1} \in C^{\infty}(\bar{\Omega})$; therefore such an asymptotic is equivalent to the boundary conditions

$$
u_{1,0,0,1}\left(x_{1}, 0\right)=M_{4} \alpha_{0}^{1}\left(x_{1}\right), \quad x_{1} \in\left(-\frac{\pi}{2}, \frac{\pi}{2}\right) .
$$

Substituting the expansion (260) of the function $u_{\varepsilon}$ in terms of $x$ and the expansion (246) into the problem and collecting terms in $\varepsilon \mu$, we conclude that

$$
\widehat{\lambda}_{\varepsilon}=\varepsilon \lambda_{1,0,0,1}+\cdots
$$

and the problem for the function $u_{1,0,0,1}$ has the form

$$
\left\{\begin{array}{l}
-\Delta u_{1,0,0,1}=\lambda_{0} u_{1,0,0,1}+\lambda_{1,0,0,1} u_{0} \quad \text { for } x \in \Omega, \\
u_{1,0,0,1}=M_{4} \alpha_{0}^{1}\left(x_{1}\right) \quad \text { for } x \in \Gamma_{4}, \\
\frac{\partial u_{1,0,0,1}}{\partial \nu}=0 \text { for } x \in \Gamma_{1} \cup \Gamma_{2} \cup \Gamma_{3} .
\end{array}\right.
$$

From the condition that this problem is solvable we obtain a formula for $\lambda_{1,0,0,1}$. We multiply the equation by $u_{0}$, integrate over the domain $\Omega$, recalling the normalization of $u_{0}$ in $L_{2}(\Omega)$, and apply Green's formula twice to the left-hand side of the equality. We obtain

$$
\lambda_{1,0,0,1}=-M_{4} \int_{-\pi / 2}^{\pi / 2} \alpha_{0}^{1^{2}}\left(x_{1}\right) d x_{1} .
$$

We now recall the form of the constant $M_{4}$ (see (253)) and substitute it into this expression. Finally we obtain

$$
\lambda_{1,0,0,1}=-\frac{\lambda_{0}}{\pi} \int_{\Gamma_{4}}\left(\frac{\partial u_{0}}{\partial \nu}\right)^{2} d s \int_{B} Y^{2}(\zeta) d \zeta .
$$

In order that (262) be uniquely solvable we will assume that

$$
\int_{\Omega} u_{0}(x) u_{1,0,0,1}(x) d x=0 .
$$


Next, in just the same way as we proceeded before, we construct the terms $v_{j, k, l, 1}$, $w_{j, k, l, 1}, u_{j, k, l, 1}$, and $\lambda_{j, k, l, 1}$ in three steps. Their appearance immediately implies that the terms $v_{j, k, l, 2}, w_{j, k, l, 2}, u_{j, k, l, 2}$, and $\lambda_{j, k, l, 2}$ appear. The way we construct them also repeats the way we constructed the terms for $\mu^{0}$ in the preceding sections. Then we construct the terms for $\mu^{3}, \mu^{4}$, and so on. As a result we obtain that the following assertion holds.

We set

$$
\begin{aligned}
\lambda_{j, k, l, 0}:=\lambda_{j, k, l}, \quad u_{j, k, l, 0}:=u_{j, k, l}, \\
v_{j, k, l, 0}:=v_{j, k, l}, \quad \text { and } \quad w_{j, k, l, 0}:=w_{j, k, l} .
\end{aligned}
$$

Theorem 3.9. There exist series

$$
\begin{aligned}
\lambda_{\varepsilon}= & \lambda_{0}+\sum_{p=0}^{\infty} \sum_{j=1}^{\infty} \sum_{l=2}^{\infty} \sum_{k=0}^{j-l+1} \varepsilon^{j} \ln ^{k} a a^{l} \mu^{p} \lambda_{j, k, l, p} \\
& +\sum_{p=0}^{\infty} \sum_{j=1}^{\infty} \sum_{k=0}^{j} \varepsilon^{j} \ln ^{k} a \mu^{p} \lambda_{j, k, 0, p}, \\
u_{\varepsilon}(x)= & u_{0}(x)+\sum_{p=0}^{\infty} \sum_{j=1}^{\infty} \sum_{l=2}^{\infty} \sum_{k=0}^{j-l+1} \varepsilon^{j} \ln ^{k} a a^{l} \mu^{p} u_{j, k, l, p}(x) \\
& +\sum_{p=0}^{\infty} \sum_{j=1}^{\infty} \sum_{k=0}^{j} \varepsilon^{j} \ln ^{k} a \mu^{p} u_{j, k, 0, p}(x), \\
u_{\varepsilon}(x)= & \sum_{p=0}^{\infty} \sum_{j=1}^{\infty} \sum_{l=2}^{\infty} \sum_{k=0}^{j-l+1} \varepsilon^{j} \ln ^{k} a a^{l} \mu^{p} v_{j, k, l, p}\left(\xi ; x_{1}\right) \\
& +\sum_{p=0}^{\infty} \sum_{j=1}^{\infty} \sum_{k=0}^{j} \varepsilon^{j} \ln ^{k} a \mu^{p} v_{j, k, 0, p}\left(\xi ; x_{1}\right) \\
& +\sum_{p=0}^{\infty} \sum_{j=1}^{\infty} \sum_{k=0}^{\infty} \varepsilon^{j} \ln ^{k} a \mu^{p} w_{j, k, 0, p}\left(\zeta ; x_{1}\right) \\
u_{\varepsilon}(x)= & \sum_{p=0}^{j} \sum_{j=1}^{\infty} \sum_{l=1}^{j} \varepsilon_{k=0}^{j} \ln ^{k} a a^{l} \mu^{p} w_{j, k, l, p}\left(\zeta ; x_{1}\right) \\
&
\end{aligned}
$$

such that

a) The pairs $u_{j, k, l, p} \in C^{\infty}(\bar{\Omega})$ and $\lambda_{j, k, l, p}$ are solutions of the problems

$$
\left\{\begin{array}{l}
-\Delta u_{j, k, l, p}=\sum_{j_{1}, k_{1}, l_{1}, p_{1}} \lambda_{j_{1}, k_{1}, l_{1}, p_{1}} u_{j-j_{1}, k-k_{1}, l-l_{1}, p-p_{1}} \quad \text { in } \Omega \\
\frac{\partial u_{j, k, l, p}}{\partial \nu}=0 \quad \text { on } \Gamma_{1} \cup \Gamma_{2} \cup \Gamma_{3} .
\end{array}\right.
$$

Henceforth we assume that the indices $j_{1}, k_{1}, l_{1}$, and $p_{1}$ vary only within the limits indicated in the corresponding sums (264), (265), (266), and (267); here $u_{0,0,0,0}=u_{0}$ and $\lambda_{0,0,0,0}=\lambda_{0}$. 
b) The series (265) has an asymptotic expansion as $x_{2} \rightarrow 0$, rewritten in terms of the variable $\xi$, of the form

$$
\begin{aligned}
u_{\varepsilon}(x)= & \sum_{p=0}^{\infty} \sum_{j=1}^{\infty} \sum_{l=2}^{\infty} \sum_{k=0}^{j-l+1} \varepsilon^{j} \ln ^{k} a a^{l} \mu^{p} V_{j, k, l, p}\left(\xi_{2} ; x_{1}\right) \\
& +\sum_{p=0}^{\infty} \sum_{j=1}^{\infty} \sum_{k=0}^{j} \varepsilon^{j} \ln ^{k} a \mu^{p} V_{j, k, 0, p}\left(\xi_{2} ; x_{1}\right) \quad \text { as } \quad \varepsilon \xi_{2} \rightarrow 0
\end{aligned}
$$

where the $V_{j, k, l, p}\left(\xi_{2} ; x_{1}\right)$ are polynomials of order $j-k$ in the variable $\xi_{2}$ whose coefficients are functions of $x_{1}$ that are infinitely differentiable on $[-\pi / 2, \pi / 2]$ and whose odd derivatives vanish at $x_{1}= \pm \pi / 2$.

c) The coefficient $\lambda_{1,1,0,0}$ is determined by the formula

$$
\lambda_{1,1,0,0}=\int_{\Gamma_{4}}\left(\frac{\partial u_{0}}{\partial \nu}\right)^{2} d s>0
$$

d) The coefficient $\lambda_{1,0,0,1}$ is given by the formula

$$
\lambda_{1,0,0,1}=-\frac{\lambda_{0}}{\pi} \int_{\Gamma_{4}}\left(\frac{\partial u_{0}}{\partial \nu}\right)^{2} d s \int_{B} Y^{2}(\zeta) d \zeta
$$

where $Y$ is the function defined in (42).

e) The functions $v_{j, k, l, p}$ can be represented as the sums

$$
v_{j, k, l, p}=V_{j, k, l, p}+\widetilde{v}_{j, k, l, p}, \quad \widetilde{v}_{j, k, l, p} \in \mathbb{A}^{(j-k, l, 1)}, \quad v_{j, j, 0,0}=\mathcal{M}_{j, j},
$$

and are solutions of the problem

$$
\left\{\begin{aligned}
&-\Delta_{\xi} v_{j, k, l, p}= 2 \frac{\partial^{2} v_{j-1, k, l, p}}{\partial x_{1} \partial \xi_{1}}+\frac{\partial^{2} v_{j-2, k, l, p}}{\partial x_{1}^{2}} \\
&+\sum_{j_{1}, k_{1}, l_{1}, p_{1}} \lambda_{j_{1}, k_{1}, l_{1}, p_{1}} v_{j-j_{1}-2, k-k_{1}, l-l_{1}, p-p_{1}} \text { in } \Pi \\
& \frac{\partial v_{j, k, l, p}}{\partial \xi_{2}}=0 \quad \text { on } \Sigma \\
& \frac{\partial v_{j, k, l, p}}{\partial \xi_{1}}\left(\xi ; \pm \frac{\pi}{2}\right)=0 \quad \text { at } \xi_{1}= \pm \frac{\pi}{2}
\end{aligned}\right.
$$

furthermore,

$\frac{\partial v_{j, k, l, p}}{\partial x_{1}}\left(\xi ; \pm \frac{\pi}{2}\right) \equiv 0 \quad$ and $\quad \frac{d^{2 l+1} \mathcal{M}_{j, j}}{d x_{1}^{2 l+1}}\left( \pm \frac{\pi}{2}\right)=0, \quad l=0,1, \ldots$

f) The functions $v_{j, k, l, p}$ have the asymptotic behaviour

$$
v_{j, k, l, p}=\mathcal{V}_{j, k, l, p} \quad \text { as } \quad \rho \rightarrow 0,
$$

where $\mathcal{V}_{j, k, l, p} \in \mathcal{F}^{(l-j+k)}$. 
g) The functions $w_{k, j, l, p} \in \mathbb{B}^{j-k, l, 1}$ are solutions of the problems

$$
\left\{\begin{aligned}
&-\Delta_{\zeta} w_{j, k, l, p}= 2 \frac{\partial^{2} w_{j-1, k, l-1, p}}{\partial x_{1} \partial \zeta_{1}}+\frac{\partial^{2} w_{j-2, k, l-2, p}}{\partial x_{1}^{2}} \\
&+\left\{\begin{array}{l}
\sum_{j_{1}, k_{1}, l_{1}, p_{1}} \lambda_{j_{1}, k_{1}, l_{1}, p_{1}} w_{j-j_{1}-2, k-k_{1}, l-l_{1}-2, p-p_{1}} \quad \text { in } \overline{\mathbb{R}_{+}^{2} \backslash B,} \\
\sum_{j_{1}, k_{1}, l_{1}, p_{1}} \lambda_{j_{1}, k_{1}, l_{1}, p_{1}} w_{j-j_{1}, k-k_{1}, l-l_{1}, p-p_{1}-1} \quad \text { in } B,
\end{array}\right. \\
& w_{j, k, l, p}=0 \quad \text { on } \gamma, \\
& \frac{\partial w_{j, k, l, p}}{\partial \zeta_{2}}=0 \quad \text { on } \Gamma .
\end{aligned}\right.
$$

h) The series (267) has an asymptotic expansion as $\tau \rightarrow+\infty$, rewritten in terms of the variables $\xi$, of the form

$$
\begin{aligned}
u_{\varepsilon}(x)= & \sum_{p=0}^{\infty} \sum_{j=1}^{\infty} \sum_{l=2}^{\infty} \sum_{k=0}^{j-l+1} \varepsilon^{j} \ln ^{k} a a^{l} \mu^{p} \mathcal{V}_{j, k, l, p}\left(\xi ; x_{1}\right) \\
& +\sum_{p=0}^{\infty} \sum_{j=1}^{\infty} \sum_{k=0}^{j} \varepsilon^{j} \ln ^{k} a \mu^{p} \mathcal{V}_{j, k, 0, p}\left(\xi ; x_{1}\right) \quad \text { as } \quad \rho a^{-1} \rightarrow+\infty
\end{aligned}
$$

\section{Substantiation of the asymptotics that have been constructed}

Let $\mathcal{N}=\left(\mathcal{N}_{1}, \mathcal{N}_{2}, \mathcal{N}_{3}\right)$ be the vector of indices, $\mathcal{N}_{s} \in \mathbb{N}$. We denote partial sums of the series (264), (265), (266), and (267), respectively, by

$$
\begin{aligned}
\tilde{\lambda}_{\varepsilon}^{\mathcal{N}}= & \lambda_{0}+\sum_{p=0}^{\mathcal{N}_{3}} \sum_{j=1}^{\mathcal{N}_{1}} \sum_{l=2}^{\mathcal{N}_{2}} \sum_{k=0}^{j-l+1} \varepsilon^{j} \ln ^{k} a a^{l} \mu^{p} \lambda_{j, k, l, p} \\
+ & \sum_{p=0}^{\mathcal{N}_{1}} \sum_{j=1}^{\mathcal{N}_{3}} \sum_{k=0}^{j} \varepsilon^{j} \ln ^{k} a \mu^{p} \lambda_{j, k, 0, p} ; \\
u_{\varepsilon}^{\mathcal{N}}(x)= & u_{0}(x)+\sum_{p=0}^{\mathcal{N}_{3}} \sum_{j=1}^{\mathcal{N}_{1}} \sum_{l=2}^{\mathcal{N}_{2}} \sum_{k=0}^{j-l+1} \varepsilon^{j} \ln ^{k} a a^{l} \mu^{p} u_{j, k, l, p}(x) \\
+ & \sum_{p=0}^{\mathcal{N}_{3}} \sum_{j=1}^{\mathcal{N}_{1}} \sum_{k=0}^{j} \varepsilon^{j} \ln ^{k} a \mu^{p} u_{j, k, 0, p}(x) ; \\
v_{\varepsilon}^{\mathcal{N}}\left(\xi ; x_{1}\right)= & \sum_{p=0}^{\mathcal{N}_{3}} \sum_{j=1}^{\mathcal{N}_{1}} \sum_{l=2}^{\mathcal{N}_{2}} \sum_{k=0}^{j-l+1} \varepsilon^{j} \ln ^{k} a a^{l} \mu^{p} v_{j, k, l, p}\left(\xi ; x_{1}\right) \\
& +\sum_{p=0}^{\mathcal{N}_{3}} \sum_{j=1}^{\mathcal{N}_{1}} \sum_{k=0}^{j} \varepsilon^{j} \ln ^{k} a \mu^{p} v_{j, k, 0, p}\left(\xi ; x_{1}\right) ; \\
w_{\varepsilon}^{\mathcal{N}}\left(\zeta ; x_{1}\right)= & \sum_{p=0}^{\mathcal{N}_{3}} \sum_{j=1}^{\mathcal{N}_{1}} \sum_{l=1}^{\mathcal{N}_{2}} \sum_{k=0}^{j-l} \varepsilon^{j} \ln ^{k} a a^{l} \mu^{p} w_{j, k, l, p}\left(\zeta ; x_{1}\right) \\
& +\sum_{p=0}^{\mathcal{N}_{3}} \sum_{j=1}^{\mathcal{N}_{1}} \sum_{k=0}^{j-1} \varepsilon^{j} \ln ^{k} a \mu^{p} w_{j, k, 0, p}\left(\zeta ; x_{1}\right) .
\end{aligned}
$$


Let $0<\beta<1$ and $\Upsilon>0$ be arbitrary numbers, and $\chi(t)$ a cutoff function, $0<\chi<1$, that is equal to 0 for $t<1$ and to 1 for $t>2$. We define

$$
\begin{gathered}
\chi_{\beta, \Upsilon}(x)=\chi\left(\frac{x_{2}}{\Upsilon \varepsilon^{\beta}}\right), \quad \chi_{\beta, \Upsilon}^{\mathbf{n}}(x)=\chi\left(\frac{\sqrt{\left(x_{1}-\mathbf{n} \pi a\right)^{2}+x_{2}^{2}}}{\Upsilon \varepsilon^{\beta} a^{\beta}}\right), \\
w_{\varepsilon}^{\mathbf{n}, \mathcal{N}}\left(\frac{x}{a \varepsilon} ; x_{1}\right)=w_{\varepsilon}^{\mathcal{N}}\left(\frac{x_{1}}{a \varepsilon}-\frac{\mathbf{n} \pi}{\varepsilon}, \frac{x_{2}}{a \varepsilon} ; x_{1}\right), \quad \mathbf{n} \in \mathbb{Z} .
\end{gathered}
$$

Lemma 4.1. The function

$$
\begin{aligned}
\widetilde{u}_{\varepsilon}^{\mathcal{N}}(x)= & u_{\varepsilon}^{\mathcal{N}} \chi_{\beta, \Upsilon}(x)+v_{\varepsilon}^{\mathcal{N}}\left(\frac{x}{\varepsilon} ; x_{1}\right)\left(1-\chi_{\beta, \Upsilon}(x)\right) \prod_{\mathbf{n}=-\mathbf{N}}^{\mathbf{N}} \chi_{\beta, \Upsilon}^{\mathbf{n}}(x) \\
& +\sum_{\mathbf{n}-\mathbf{N}}^{\mathbf{N}} w_{\varepsilon}^{\mathbf{n}, \mathcal{N}}\left(\frac{x}{a \varepsilon} ; x_{1}\right)\left(1-\chi_{\beta, \Upsilon}^{\mathbf{n}}(x)\right)
\end{aligned}
$$

is an $H^{1}(\Omega)$ solution of the boundary-value problem

$$
\left\{\begin{array}{l}
-\Delta \widetilde{u}_{\varepsilon}^{\mathcal{N}}=\widetilde{\lambda}_{\varepsilon}^{\mathcal{N}} \rho_{\varepsilon} \widetilde{u}_{\varepsilon}^{\mathcal{N}}+f_{\varepsilon}^{\mathcal{N}} \quad \text { in } \Omega \\
\widetilde{u}_{\varepsilon}^{\mathcal{N}}=0 \quad \text { on } \gamma_{\varepsilon}, \\
\frac{\partial \widetilde{u}_{\varepsilon}^{\mathcal{N}}}{\partial \nu}=0 \quad \text { on } \Gamma_{\varepsilon} \cup \Gamma_{1} \cup \Gamma_{2} \cup \Gamma_{3},
\end{array}\right.
$$

where $\rho_{\varepsilon}$ is given by formula (3),

$$
\left\|f_{\varepsilon}^{\mathcal{N}}\right\|_{0}=O\left(\varepsilon^{\mathcal{N}_{4}} a^{\mathcal{N}_{5}} \mu^{\mathcal{N}_{6}}\right)
$$

and the constants $\mathcal{N}_{4}, \mathcal{N}_{5}$, and $\mathcal{N}_{6}$ can be explicitly calculated and satisfy

$$
\mathcal{N}_{s} \rightarrow+\infty, \quad s=4,5,6 \quad \text { as } \quad \mathcal{N}_{q} \rightarrow+\infty, \quad q=1,2,3 .
$$

Furthermore,

$$
\widetilde{u}_{\varepsilon}^{\mathcal{N}} \longrightarrow u_{0} \quad \text { strongly in } L_{2}(\Omega) \quad \text { as } \quad a, \varepsilon, \mu \rightarrow 0
$$

Proof. Using the boundary conditions for the problems (268), (270), and (272), the function $\widetilde{u}_{\varepsilon}^{\mathcal{N}}$ satisfies the boundary conditions for (279). Acting on the function $\widetilde{u}_{\varepsilon}^{\mathcal{N}}$ by the operator $\left(-\Delta-\widetilde{\lambda}_{\varepsilon}^{\mathcal{N}} \rho_{\varepsilon}\right)$ we find that

$$
f_{\varepsilon}^{\mathcal{N}}=I_{1}+I_{2}+I_{3}+I_{4}+I_{5},
$$


where

$$
\begin{aligned}
I_{1}= & \chi_{\beta, \Upsilon}\left(-\Delta u_{\varepsilon}^{\mathcal{N}}-\widetilde{\lambda}_{\varepsilon}^{\mathcal{N}} u_{\varepsilon}^{\mathcal{N}}\right), \\
I_{2}= & \left(1-\chi_{\beta, \Upsilon}\right) \prod_{\mathbf{n}=-\mathbf{N}}^{\mathbf{N}} \chi_{\beta, \Upsilon}^{\mathbf{n}}\left(-\Delta v_{\varepsilon}^{\mathcal{N}}-\widetilde{\lambda}_{\varepsilon}^{\mathcal{N}} \rho_{\varepsilon} v_{\varepsilon}^{\mathcal{N}}\right), \\
I_{3}= & \sum_{\mathbf{n}=-\mathbf{N}}^{\mathbf{N}}\left(-\Delta w_{\varepsilon}^{\mathbf{n}, \mathcal{N}}-\widetilde{\lambda}_{\varepsilon}^{\mathcal{N}} w_{\varepsilon}^{\mathbf{n}, \mathcal{N}}\right)\left(1-\chi_{\beta, \Upsilon}^{\mathbf{n}}\right), \\
I_{4}= & 2 \frac{1}{\Upsilon \varepsilon^{\beta}} \frac{d \chi}{d t}\left(\frac{\partial v_{\varepsilon}^{\mathcal{N}}}{\partial x_{2}}-\frac{\partial u_{\varepsilon}^{\mathcal{N}}}{\partial x_{2}}\right) \\
& +\frac{1}{\Upsilon^{2} \varepsilon^{2 \beta}} \frac{d^{2} \chi}{d t^{2}}\left(v_{\varepsilon}^{\mathcal{N}}-u_{\varepsilon}^{\mathcal{N}}\right), \\
I_{5}= & -2 \nabla v_{\varepsilon}^{\mathcal{N}}\left(1-\chi_{\beta, \Upsilon}\right) \nabla\left(\prod_{\mathbf{n}=-\mathbf{N}}^{\mathbf{N}} \chi_{\beta, \Upsilon}^{\mathbf{n}}\right) \\
& -v_{\varepsilon}^{\mathcal{N}}\left(1-\chi_{\beta, \Upsilon}\right) \Delta\left(\prod_{\mathbf{n}=-\mathbf{N}}^{\mathbf{N}} \chi_{\beta, \Upsilon}^{\mathbf{n}}\right) \\
& +2 \sum_{\mathbf{n}=-\mathbf{N}}^{\mathbf{N}} \nabla w_{\varepsilon}^{\mathbf{n}, \mathcal{N}} \nabla \chi_{\beta, \Upsilon}^{\mathbf{n}}+\sum_{\mathbf{n}=-2 \mathbf{N}}^{2 \mathbf{N}} w_{\varepsilon}^{\mathbf{n}, \mathcal{N}} \Delta \chi_{\beta, \Upsilon}^{\mathbf{n}} .
\end{aligned}
$$

It follows from parts a), e), and g) of Theorem 3.9 that

$$
\begin{gathered}
\left\|I_{s}\right\|=O\left(\varepsilon^{\mathcal{N}_{4}} a^{\mathcal{N}_{5}} \mu^{\mathcal{N}_{6}}\right), \\
\mathcal{N}_{p} \rightarrow+\infty, \quad p=4,5,6 \quad \text { as } \quad \mathcal{N}_{q} \rightarrow+\infty, \quad q=1,2,3,
\end{gathered}
$$

for $s=1,2,3$, respectively. We observe that the support of $I_{4}$ is contained in the layer $\Upsilon \varepsilon^{\beta}<x_{2}<2 \Upsilon \varepsilon^{\beta}$, while the support of $I_{5}$ is contained in the union of the half-rings

$$
\begin{gathered}
\Upsilon a^{\beta} \varepsilon^{\beta}<\sqrt{\left(x_{1}-\mathbf{n} \pi a\right)^{2}+x_{2}^{2}}<\Upsilon a^{\beta} \varepsilon^{\beta} \\
\mathbf{n} \in \mathbb{Z}, \quad-\mathbf{N} \leq \mathbf{n} \leq \mathbf{N}
\end{gathered}
$$

there are of the order of $\varepsilon^{-1}$ of these. This fact and parts b), e) and f), h) of Theorem 3.9 imply that the estimate (285) holds for $s=4$ and 5, respectively. The estimate (280) follows from (283) and (285) for $s=1,2,3,4,5$. The limit (282) follows from the definition of $\widetilde{u}_{\varepsilon}^{\mathcal{N}}$. The lemma is proved.

We have thus completed the construction of the formal asymptotics of the eigenvalues and eigenfunctions. We now pass to a rigorous substantiation of the asymptotics we have constructed. The following statement holds. 
Theorem 4.1. The asymptotic of the eigenvalues and the corresponding eigenfunctions has the form

$$
\begin{gathered}
\lambda_{\varepsilon}=\lambda_{0}+\sum_{p=0}^{\infty} \sum_{j=1}^{\infty} \sum_{l=2}^{\infty} \sum_{k=0}^{j-l+1} \varepsilon^{j} \ln ^{k} a a^{l} \mu^{p} \lambda_{j, k, l, p} \\
+\sum_{p=0}^{\infty} \sum_{j=1}^{\infty} \sum_{k=0}^{j} \varepsilon^{j} \ln ^{k} a \mu^{p} \lambda_{j, k, 0, p}, \\
\mathcal{U}_{\varepsilon}(x)=u_{0}(x)+\sum_{p=0}^{\infty} \sum_{j=1}^{\infty} \sum_{l=2}^{\infty} \sum_{k=0}^{j-l+1} \varepsilon^{j} \ln ^{k} a a^{l} \mu^{p} u_{j, k, l, p}(x) \\
+\sum_{p=0}^{\infty} \sum_{j=1}^{\infty} \sum_{k=0}^{j} \varepsilon^{j} \ln ^{k} a \mu^{p} u_{j, k, 0, p}(x) \quad \text { for } x_{2} \geq \varepsilon^{\beta}, \\
\mathcal{U}_{\varepsilon}(x)=\sum_{p=0}^{\infty} \sum_{j=1}^{\infty} \sum_{l=2}^{\infty} \sum_{k=0}^{j-l+1} \varepsilon^{j} \ln ^{k} a a^{l} \mu^{p} v_{j, k, l, p}\left(\frac{x}{\varepsilon} ; x_{1}\right) \\
+\sum_{p=0}^{\infty} \sum_{j=1}^{\infty} \sum_{k=0}^{j} \varepsilon^{j} \ln ^{k} a \mu^{p} v_{j, k, 0, p}\left(\frac{x}{\varepsilon} ; x_{1}\right) \\
\quad f o r x_{2}<2 \varepsilon^{\beta},\left(x_{1}-a \mathbf{n} \pi\right)^{2}+x_{2}^{2} \geq \varepsilon^{2 \beta} a^{2 \beta}, \\
\mathcal{U}_{\varepsilon}(x)=\sum_{p=0}^{\infty} \sum_{j=1}^{\infty} \sum_{l=1}^{\infty} \sum_{k=0}^{j-l} \varepsilon^{j} \ln ^{k} a a^{l} \mu^{p} w_{j, k, l, p}\left(\frac{x_{1}}{a \varepsilon}-\frac{\mathbf{n} \pi}{\varepsilon}, \frac{x_{2}}{a \varepsilon} ; x_{1}\right) \\
+\sum_{p=0}^{\infty} \sum_{j=1}^{\infty} \sum_{k=0}^{j-1} \varepsilon^{j} \ln ^{k} a \mu^{p} w_{j, k, 0, p}\left(\frac{x_{1}}{a \varepsilon}-\frac{\mathbf{n} \pi}{\varepsilon}, \frac{x_{2}}{a \varepsilon} ; x_{1}\right) \\
\text { for }\left(x_{1}-a \mathbf{n} \pi\right)^{2}+x_{2}^{2}<2 \varepsilon^{2 \beta} a^{2 \beta}, \quad \mathbf{n} \in \mathbb{Z}, \quad-\mathbf{N} \leq \mathbf{n} \leq \mathbf{N},
\end{gathered}
$$

in the norm of the Sobolev space $H^{1}(\Omega)$. Here the $u_{j, k, l, p}, \lambda_{j, k, l, p}, v_{j, k, l, p}$, and $w_{j, k, l, p}$ satisfy the conditions of Theorem 3.9 and, in particular,

$$
\lambda_{1,1,0,0}=\int_{-\pi / 2}^{\pi / 2}\left(\alpha_{0}^{1}\right)^{2}\left(x_{1}\right) d x_{1}>0, \quad \lambda_{1,0,0,1}=-\frac{\lambda_{0}}{\pi} \int_{-\pi / 2}^{\pi / 2}\left(\alpha_{0}^{1}\right)^{2}\left(x_{1}\right) d x_{1} \int_{B} Y^{2}(\zeta) d \zeta
$$

where

$$
Y(\zeta)=\operatorname{Re} \ln \left(y+\sqrt{y^{2}-1}\right),
$$

$y=\zeta_{1}+\mathrm{i} \zeta_{2}, \quad w_{1,0,0,0}\left(\zeta ; x_{1}\right)=\alpha_{0}^{1}\left(x_{1}\right) Y(\zeta), \quad v_{1,0,0,0}\left(\xi ; x_{1}\right)=\alpha_{0}^{1}\left(x_{1}\right) X(\xi)$, where $X(\xi)=$ $\operatorname{Re} \ln \sin z+\ln 2, \quad z=\xi_{1}+\mathrm{i} \xi_{2}, \quad \alpha_{0}^{1}\left(x_{1}\right)=\left.\frac{\partial u_{0}}{\partial x_{2}}\right|_{x_{2}=0}$.

Remark 4.1. We point out that in the theorem an asymptotic of a non-normalized eigenfunction is constructed, that is, of $\mathcal{U}_{\varepsilon}(x)=T_{\varepsilon} u_{\varepsilon}(x)$, with the constant satisfy$\operatorname{ing} T_{\varepsilon}=1+o(1)$ as $\varepsilon \rightarrow 0$, where $u_{\varepsilon}$ is an eigenfunction of the problem (2) normalized in $L_{2}(\Omega)$.

Proof. Consider the function $\widetilde{u}_{\varepsilon}^{\mathcal{N}}$ defined in (279). Taking the fact that $\left\|\widetilde{u}_{\varepsilon}^{\mathcal{N}}\right\|_{0} \rightarrow 1$ as $\varepsilon \rightarrow 0$ into account and applying Theorem 2.3 (part 1) with $N=1$ ) for $\lambda=\widetilde{\lambda}_{\varepsilon}^{\mathcal{N}}$, $U_{\varepsilon}=\widetilde{u}_{\varepsilon}^{\mathcal{N}}$, and $f=f_{\varepsilon}^{\mathcal{N}}$, we conclude that

$$
\left|\widetilde{\lambda}_{\varepsilon}^{\mathcal{N}}-\lambda_{\varepsilon}\right|=O\left(\varepsilon^{\mathcal{N}_{4}} a^{\mathcal{N}_{5}} \mu^{\mathcal{N}_{6}}\right) .
$$

As the choice of $\mathcal{N}$ is arbitrary, using condition (281), the asymptotics of $\lambda_{\varepsilon}$ have the form (286). We pass to substantiating the asymptotics constructed for the eigenfunction. 
Recall that by the theorem on convergence, $u_{\varepsilon} \rightarrow u_{0}$ strongly in $L_{2}(\Omega)$ as $\varepsilon \rightarrow 0$. Consequently, by (282) we have

$$
\left\|\widetilde{u}_{\varepsilon}^{\mathcal{N}}-u_{\varepsilon}\right\|_{0} \longrightarrow 0 \quad \text { as } \quad \varepsilon \rightarrow 0
$$

where $u_{\varepsilon}$ is a solution of the problem (2). The representation $\widetilde{u}_{\varepsilon}^{\mathcal{N}}=u_{\varepsilon} \int_{\Omega} u_{\varepsilon} \widetilde{u}_{\varepsilon}^{\mathcal{N}} d x+u_{\varepsilon}^{\perp}$ with $u_{\varepsilon}^{\perp} \perp u_{\varepsilon}$ and (291) imply that

$$
\widetilde{u}_{\varepsilon}^{\mathcal{N}}=T_{\varepsilon}^{\mathcal{N}} u_{\varepsilon}+u_{\varepsilon}^{\perp}, \quad T_{\varepsilon}^{\mathcal{N}}=1+o(1) .
$$

It follows from (290), (292), and Lemma 4.1 that the function $u_{\varepsilon}^{\perp}$ is a solution of the boundary-value problem

$$
\left\{\begin{array}{l}
-\Delta u_{\varepsilon}^{\perp}=\widetilde{\lambda}_{\varepsilon}^{\mathcal{N}} \rho_{\varepsilon} u_{\varepsilon}^{\perp}+F_{\varepsilon}^{\mathcal{N}} \quad \text { for } x \in \Omega, \\
u_{\varepsilon}^{\perp}=0 \text { for } x \in \gamma_{\varepsilon}, \\
\frac{\partial u_{\varepsilon}^{\perp}}{\partial \nu}=0 \quad \text { for } x \in \Gamma_{\varepsilon} \cup \Gamma_{1} \cup \Gamma_{2} \cup \Gamma_{3},
\end{array}\right.
$$

where

$$
F_{\varepsilon}^{\mathcal{N}}=f_{\varepsilon}^{\mathcal{N}}+\rho_{\varepsilon} T_{\varepsilon} u_{\varepsilon}\left(\widetilde{\lambda}_{\varepsilon}^{\mathcal{N}}-\lambda_{\varepsilon}\right)=O\left(\varepsilon^{\mathcal{N}_{4}-m} a^{\mathcal{N}_{5}-m} \mu^{\mathcal{N}_{6}}\right) .
$$

It follows from (293), (294), and part 2) of Theorem 2.3 with $N=1$ for $\lambda=\widetilde{\lambda}_{\varepsilon}^{\mathcal{N}}$, $U_{\varepsilon}=\widetilde{u}_{\varepsilon}^{\mathcal{N}}$, and $f=f_{\varepsilon}^{\mathcal{N}}$ that

$$
\left\|u_{\varepsilon}^{\perp}\right\|_{0}=O\left(\varepsilon^{\mathcal{N}_{4}-m} a^{\mathcal{N}_{5}-m} \mu^{\mathcal{N}_{6}}\right) .
$$

Finally, it follows from (292), (295), from the fact that the choice of $\mathcal{N}, \Upsilon$ is arbitrary, and from (281) that the asymptotic behaviour of the eigenfunction has the form (287), (288), (289). Thus, the theorem is proved.

Remark 4.2. When $a=\varepsilon^{h}$ and in addition $(2 h+1) /(h+1)<m<2$, the correction (263) comes second in the asymptotic expansion of the eigenvalue, that is,

$$
\lambda_{\varepsilon}=\lambda_{0}+\varepsilon \ln a \lambda_{1,1,0,0}+\varepsilon \mu \lambda_{1,0,0,1}+\cdots
$$

and the other terms have lower order.

\section{Appendix 1. AuXiliary RESUlts For the CONSTRUCtion OF THE 'INTERMEDIATE' EXPANSION}

Proof of Lemma 3.1. It follows from the definition (28) and from (30) that

$$
\frac{\partial X}{\partial \xi_{1}}=A \rho^{-1} \cos \theta+\sum_{j=0}^{\infty} \widetilde{B}_{2 j} \rho^{2 j+1} \cos (2 j+1) \theta \quad \text { as } \quad \rho \rightarrow 0 .
$$

Let $Z_{j}(\xi)$ be an arbitrary harmonic polynomial of order $j$. It is known (see, for example, 69]) that the equations

$$
\Delta u=Z_{j} \rho^{\alpha}, \quad \alpha \neq-2 j-2, \quad \alpha \neq-2, \quad \Delta u=Z_{j} \rho^{-2}
$$

have solutions of the form $u=a_{1} Z_{j} \rho^{\alpha+2}$ and $u=a_{2} Z_{j} \ln \rho$, respectively. The constants $a_{1}$ and $a_{2}$ can be calculated explicitly. It follows from this fact and from (296) that there exists a series

$$
\widehat{X}_{2}(\xi)=Q \rho \ln \rho \cos \theta+\sum_{j=0}^{\infty} \beta_{2 j+1} \rho^{2 j+3} \cos (2 j+1) \theta
$$

(where $Q, \beta_{k}$ are constants that can be calculated explicitly) that is a formal asymptotic solution of the equation

$$
\Delta \widehat{X}_{2}=-2 \frac{\partial X}{\partial \xi_{1}} \quad \text { as } \rho \rightarrow 0
$$


We set

$$
\widehat{X}_{2}^{N}(\xi)=Q \rho \ln \rho \cos \theta+\sum_{j=0}^{N} \beta_{2 j+1} \rho^{2 j+3} \cos (2 j+1) \theta
$$

in $\Pi$ and extend $(1-\chi(\rho)) \widehat{X}_{2}^{N}(\xi)$ periodically in $\xi_{1}$ to $\mathbb{R}_{+}^{2}$. We seek $X_{2}(\xi)$ in the form

$$
X_{2}(\xi)=(1-\chi(\rho)) \widehat{X}_{2}^{N}(\xi)+\breve{X}_{2}(\xi)
$$

in $\Pi$, also extending $\breve{X}_{2}(\xi)$ periodically in $\xi_{1}$ to $\mathbb{R}_{+}^{2}$. By (52), for the function $\breve{X}_{2}$ we obtain the boundary-value problem

$$
\left\{\begin{array}{l}
-\Delta_{\xi} \breve{X}_{2}=F \quad \text { in } \Pi, \\
\frac{\partial \breve{X}_{2}}{\partial \xi_{2}}=0 \quad \text { on } \breve{\Sigma} \\
\breve{X}_{2}=0 \quad \text { at } \xi_{1}= \pm \frac{\pi}{2}
\end{array}\right.
$$

where

$$
\begin{gathered}
e^{\varpi \xi_{2}} F \in L_{2}(\Pi), \\
F=O\left(\rho^{2 N+3}\right) \quad \text { as } \quad \rho \rightarrow 0 .
\end{gathered}
$$

Using (28), (299), and (300) we see that the function $F$ is odd and $\pi$-periodic in $\xi_{1}$ and

$$
F \in C^{\infty}\left(\overline{\mathbb{R}_{+}^{2}} \backslash \bigcup_{k \in \mathbb{Z}}\{(k \pi, 0)\}\right) \cap C^{2 N+3}\left(\overline{\mathbb{R}_{+}^{2}}\right) .
$$

By (302) there exists a unique solution of the problem (301) such that

$$
e^{\varpi \xi_{2}} \breve{X}_{2} \in H^{1}(\Pi)
$$

(see [74, Ch. 2,5]). Furthermore, using (304), the problem (301), and the fact that the function $F$ is odd and $\pi$-periodic in $\xi_{1}$, we conclude that

$$
\breve{X}_{2} \in C^{\infty}\left(\overline{\mathbb{R}_{+}^{2}} \backslash \bigcup_{k \in \mathbb{Z}}\{(k \pi, 0)\}\right) \cap C^{2 N+3}\left(\overline{\mathbb{R}_{+}^{2}}\right)
$$

$\breve{X}_{2}$ is an odd function in $\xi_{1}$ (by the uniqueness of the solution of the problem (301)), and by (303) it has an asymptotic expansion of the form

$$
\breve{X}_{2}=\sum_{j=0}^{N+1} \varrho_{2 j+1} \rho^{2 j+1} \cos (2 j+1) \theta+O\left(\rho^{2 N+5}\right) \quad \text { as } \rho \rightarrow 0 .
$$

Hence, all the assertions of the lemma follow when we apply (299), (300), the fact that $N$ is an arbitrary number, and that the problem (301) is uniquely solvable in the class of functions under consideration.

It is known (see, for example, 69 ) that the equation

$$
\Delta u=Z_{j} \rho^{\alpha} \ln \rho, \quad \alpha \geq 0,
$$

has a solution of the form $u=a_{3} Z_{j} \rho^{\alpha+2}+a_{4} Z_{j} \rho^{\alpha+2} \ln \rho$. The constants $a_{3}$ and $a_{4}$ can be calculated explicitly. In view of this fact, similarly to Lemma 3.1, we can prove the following assertion. 
Lemma 4.2. Let $F \in A_{\mathrm{odd}}^{(2 k-1,0)}$; then the problem

$$
\left\{\begin{array}{l}
-\Delta_{\xi} W=F \quad \text { in } \Pi, \\
W=0 \quad \text { at } \xi_{1}= \pm \frac{\pi}{2} \\
\frac{\partial W}{\partial \xi_{2}}=0 \quad \text { on } \Sigma
\end{array}\right.
$$

has a solution $W \in A_{\mathrm{odd}}^{(2 k+1,1)}$.

The definition of the class $\mathcal{A}_{\text {odd }}^{(2 j+1, q)}$ and Lemma 4.2 imply the following lemma.

Lemma 4.3. Let $F \in \mathcal{A}_{\text {odd }}^{(2 k-1,0)}$; then the problem

$$
\left\{\begin{array}{l}
-\Delta_{\xi} W=F \quad \text { in } \Pi, \\
\frac{\partial W}{\partial \xi_{2}}=0 \quad \text { on } \Sigma \\
W=0 \quad \text { at } \xi_{1}= \pm \frac{\pi}{2}
\end{array}\right.
$$

has a solution $W \in \mathcal{A}_{\text {odd }}^{(2 k+1,1)}$.

Lemma 4.4. Let $e^{\varpi \xi_{2}} F \in L_{2}(\Pi)$; then the problem

$$
\left\{\begin{array}{l}
-\Delta_{\xi} W=F \quad \text { in } \Pi, \\
\frac{\partial W}{\partial \xi_{1}}=0 \quad \text { at } \xi_{1}= \pm \frac{\pi}{2}, \\
\frac{\partial W}{\partial \xi_{2}}=0 \quad \text { on } \Sigma
\end{array}\right.
$$

has a solution $W$ that can be represented in the form

$$
W=c \xi_{2}+\widetilde{W},
$$

where $e^{\varpi \xi_{2}} \widetilde{W} \in H^{1}(\Pi)$.

Proof. We represent the function $W$ in the form of a sum

$$
W=W_{1}+W_{2},
$$

where $W_{1}$ is a solution of the problem

$$
\left\{\begin{array}{l}
-\Delta_{\xi} W_{1}=F \quad \text { in } \Pi, \\
\frac{\partial W_{1}}{\partial \xi_{1}}=0 \quad \text { at } \xi_{1}= \pm \frac{\pi}{2}, \\
W_{1}=0 \text { on } \Sigma
\end{array}\right.
$$

and $W_{2}$ solves the problem

$$
\left\{\begin{array}{l}
-\Delta_{\xi} W_{2}=0 \quad \text { in } \Pi, \\
\frac{\partial W_{2}}{\partial \xi_{1}}=0 \quad \text { at } \xi_{1}= \pm \frac{\pi}{2}, \\
\frac{\partial W_{2}}{\partial \xi_{2}}=-\frac{\partial W_{1}}{\partial \xi_{2}} \quad \text { on } \Sigma .
\end{array}\right.
$$

By the hypothesis of the lemma, the problem (312) is solvable in the class

$$
e^{\varpi \xi_{2}} W_{1} \in H^{1}(\Pi)
$$


(see [74, Ch. 2,5]). It follows from the theorem on the increase of smoothness that

$$
\frac{\partial W_{1}}{\partial \xi_{2}} \in L_{2}(\breve{\Sigma}) .
$$

Next, solving the problem (313) by the standard method of separation of variables, we find that a solution of the form $W_{2}=c \xi_{2}+\widetilde{W}_{2}$ exists, where $e^{\varpi \xi_{2}} \widetilde{W}_{2} \in H^{1}(\Pi)$. This fact and (314) prove the lemma.

Taking account of Lemma 4.4, similarly to Lemma 3.1. we can prove that the following assertion holds.

Lemma 4.5. Let $F \in A_{\mathrm{even}}^{(2 k, 0)}$; then the problem (310) has a solution $W$ that can be represented in the form

$$
W=c \xi_{2}+\widetilde{W}
$$

where $\widetilde{W} \in A_{\text {even }}^{(2 k+2,1)}, \widetilde{W}=O(1)$ as $\rho \rightarrow 0$, and $c$ is some constant.

By subtracting the function $c X(\xi)$ from (315) we obtain the following.

Corollary. Suppose that F satisfies the hypotheses of Lemma 4.5; then the problem (310) has a solution $W \in A_{\text {even }}^{(2 k+2,1)}$.

Throughout what follows, $P_{j}\left(\xi_{2}\right)$ will denote a polynomial of order $j$. The following assertion follows immediately from Lemma 4.5.

Lemma 4.6. Suppose that $F$ can be represented in the form $F=P_{t}+f$, where $f \in A_{\text {even }}^{(2 k, 0)}$ and $P_{t+2}$ is a polynomial such that $-P_{t+2}^{\prime \prime}=P_{t}$. Then there exists a solution of the boundary-value problem (310) that can be represented in the form $W=P_{t+2}+W_{1}$, where $W_{1} \in A_{\text {even }}^{(2 k+2,1)}$.

Proof of Lemma 3.2. The definitions of $\mathcal{A}_{\text {even }}^{(2 k, q)}$ and $\mathcal{P}_{t}\left(\xi_{2} ; x_{1}\right)$ and Lemma 4.6 imply the proof of part a) of Lemma 3.2. The first equality of part b) follows from the definitions of $\mathcal{A}_{\text {even }}^{(2 k, q)}$ and $\mathcal{P}_{t}\left(\xi_{2} ; x_{1}\right)$. Since

$$
\frac{\partial W}{\partial \xi_{1}}=0 \quad \text { at } \xi_{1}= \pm \frac{\pi}{2}
$$

(by part a)), the first equality implies the second equality of part b).

Proof of Theorem 3.1. The proof of part a) of the theorem follows immediately from Lemmas 4.3 and 4.6. The first equality in part b) follows from the definition of the class $\mathbb{A}^{(k+2,1)}$ and from Lemma 4.3. The first equality and the fact that $\partial W / \partial \xi_{1}(\xi ; \pm \pi / 2)=0$ at $\xi_{1}= \pm \pi / 2$ (by part a)) imply that the second equality in part b) holds.

\section{Appendix 2. Auxiliary Results For the CONSTRUCtion OF THE 'INNER' EXPANSION}

The explicit form of the Green's function for the Neumann problem for the half-plane and the theorem on the increase of smoothness yield the following result.

Lemma 4.7. Suppose that $F \in L_{2}\left(\mathbb{R}_{+}^{2}\right) \cap C^{\infty}(\widetilde{B})$ has asymptotic behaviour

$$
F=O\left(\tau^{-N-1}\right) \quad \text { as } \quad \tau \rightarrow+\infty
$$

and that $\varphi \in C_{0}^{\infty}(\gamma)$ is such that $\int_{\mathbb{R}} \varphi d \zeta_{1}=1$. Then there exists a function

$$
W_{1} \in H_{\text {loc }}^{2}\left(\mathbb{R}_{+}^{2}\right) \cap C^{\infty}(\widetilde{B})
$$


that is a solution of the boundary-value problem

$$
\left\{\begin{array}{l}
\Delta_{\zeta} W_{1}=F \quad \text { in } \mathbb{R}_{+}^{2} \\
\frac{\partial W_{1}}{\partial \zeta_{2}}=\left(\int_{\mathbb{R}_{+}^{2}} F d \zeta\right) \varphi \quad \text { at } \zeta_{2}=0
\end{array}\right.
$$

with the asymptotics

$$
W_{1}=\sum_{j=0}^{N-2} \widetilde{a}_{j} \tau^{-j} \cos (j \theta)+O\left(\tau^{-N+1}\right), \quad \tau \rightarrow \infty .
$$

Proof of Lemma 3.6. We seek a solution of the problem (250) in the form of a sum $W=W_{1}+W_{2}$, where $W_{1}$ satisfies the assertion of Lemma 4.7. Then the function $W_{2}$ must satisfy the following boundary-value problem:

$$
\left\{\begin{array}{l}
\Delta_{\zeta} W_{2}=0 \quad \text { in } \mathbb{R}_{+}^{2}, \\
\frac{\partial W_{2}}{\partial \zeta_{2}}=0 \quad \text { on } \Gamma, \\
W_{2}=-W_{1} \quad \text { on } \gamma .
\end{array}\right.
$$

It follows from Lemma 4.7 that $W_{1} \in H^{3 / 2}(\gamma) \cap C^{\infty}(\gamma)$ (and, consequently, satisfies a Hölder condition at each point of $\gamma$ ). Therefore the Keldysh-Sedov formula (see, for example, [75, Ch. III, §3]) and the theorem on the increase of smoothness imply that there exists a solution

$$
W_{2} \in H_{\text {loc }}^{1}\left(\mathbb{R}_{+}^{2}\right) \cap C^{\infty}\left(\mathbb{R}_{+}^{2} \cup \Gamma \cup \gamma\right)
$$

of the problem (318) with the asymptotics

$$
W_{2}=\sum_{j=0}^{+\infty} \widetilde{b}_{j} \tau^{-j} \cos (j \theta), \quad \tau \rightarrow \infty .
$$

This fact and the statement of Lemma 4.7 imply that the first statement in Lemma 3.6 holds. The second part of the lemma follows from the fact that the solution of problem (318) which is bounded at infinity is unique.

- We denote by $\bar{H}_{\text {odd }}^{(2 k-1)}, k=1, \ldots$, the set of polynomials in $\tau$ of the form

$$
\sum_{i=0}^{k-1} \sum_{j=0}^{i} \varkappa_{\text {odd }}^{(i, j)} \tau^{2 i+1} \cos (2 i-2 j+1) \theta
$$

with constant coefficients.

We denote by $\bar{H}_{\text {even }}^{(1,2 k)}, k=1, \ldots$, the set of polynomials in $\tau$ of the form

$$
\sum_{i=1}^{k} \sum_{j=0}^{i} \varkappa_{\text {even }}^{(i, j)} \tau^{2 i} \cos 2(i-j) \theta
$$

with constant coefficients.

We denote by $\bar{H}_{\text {even }}^{(0,2 k)}, k=0,1, \ldots$, the set of polynomials in $\tau$ of the form

$$
\sum_{i=0}^{k} \sum_{j=0}^{i} \varkappa_{\text {even }}^{(i, j)}\left(x_{1}\right) \tau^{2 i} \cos 2(i-j) \theta
$$

with constant coefficients. 
We also set

$$
\begin{aligned}
& \bar{F}_{\text {odd }}^{(2 k-1)}=\left\{V(\zeta): V(\zeta)=\sum_{j=1}^{k} \ln \tau h_{2 j-1}(\zeta)+\sum_{j=1}^{k} \widetilde{h}_{2 j-1}(\zeta), h_{2 j-1}, \widetilde{h}_{2 j-1} \in \bar{H}_{\text {odd }}^{(2 j-1)}\right\}, \\
& \bar{F}_{\text {even }}^{(1,2 k)}=\left\{V(\zeta): V(\zeta)=\sum_{j=1}^{k} \ln \tau h_{2 j}(\zeta)+\sum_{j=1}^{k} \widetilde{h}_{2 j}(\zeta), h_{2 j}, \widetilde{h}_{2 j} \in \bar{H}_{\text {even }}^{(1,2 j)}\right\} \\
& \bar{F}_{\text {even }}^{(0,2 k)}=\left\{V(\zeta): V(\zeta)=\sum_{j=0}^{k} \ln \tau h_{2 j}(\zeta)+\sum_{j=0}^{k} \widetilde{h}_{2 j}(\zeta), h_{2 j}, \widetilde{h}_{2 j} \in \bar{H}_{\text {even }}^{(0,2 j)}\right\} .
\end{aligned}
$$

Lemma 4.8. Let $F \in B_{\text {odd }}^{(2 k-1,2 n-1,0)}$ and let $Q$ be a function in $\bar{F}_{\text {odd }}^{(2 n+1)}$ such that

$$
F-\Delta Q=O\left(\tau^{-2}\right) \quad \text { as } \quad \tau \rightarrow+\infty .
$$

Then there exists a solution $W \in B_{\mathrm{odd}}^{(2 k+1,2 n+1,1)}$ of the boundary-value problem

$$
\left\{\begin{array}{l}
\Delta_{\zeta} W=F \quad \text { in } \mathbb{R}_{+}^{2}, \\
\frac{\partial W}{\partial \zeta_{2}}=0 \quad \text { on } \Gamma \\
W=0 \quad \text { on } \gamma, \\
W \sim Q \quad \text { as } \tau \rightarrow+\infty
\end{array}\right.
$$

Proof. The form of the solutions of equations (297) and (307) implies that there exists a series

$$
\widetilde{\mathcal{Q}}=\widetilde{Q}+\sum_{i=0}^{+\infty} \sum_{j=0}^{i} \widetilde{d}_{i, j}^{\text {odd }} \tau^{-2 i+2 j-1} \cos (2 i+1) \theta,
$$

where $\widetilde{Q} \in \bar{F}_{\text {odd }}^{(2 n+1)}$, that is a formal asymptotic solution as $\tau \rightarrow+\infty$ of the problem

$$
\left\{\begin{array}{l}
\Delta_{\zeta} \mathcal{Q}=F \quad \text { in } \mathbb{R}_{+}^{2}, \\
\frac{\partial \mathcal{Q}}{\partial \zeta_{2}}=0 \quad \text { at } \zeta_{2}=0
\end{array}\right.
$$

Taking account of (320) we conclude that $\Delta Q-\Delta \widetilde{Q}=O\left(\tau^{-2}\right)$. Consequently, the series

$$
\mathcal{Q}=Q+\sum_{i=0}^{+\infty} \sum_{j=0}^{i} d_{i, j}^{\text {odd }} \tau^{-2 i+2 j-1} \cos (2 i+1) \theta
$$

is also a formal asymptotic solution as $\tau \rightarrow+\infty$ of (323). We seek a solution of the boundary-value problem (321) in the form

$$
W(\zeta)=W_{N}(\zeta)=(1-\chi(\tau R)) \mathcal{Q}_{2 N+1}(\zeta)+W_{N}^{(0)}(\zeta),
$$

where $\mathcal{Q}_{2 N+1}$ is a partial sum of the series (324), and $\chi$ is the cutoff function introduced in 4 . Then for $W_{N}^{(0)}$ we obtain the boundary-value problem

$$
\left\{\begin{array}{l}
\Delta_{\zeta} W_{N}^{(0)}=F_{N} \quad \text { in } \mathbb{R}_{+}^{2}, \\
\frac{\partial W_{N}^{(0)}}{\partial \zeta_{2}}=0 \text { on } \Gamma, \\
W_{N}^{(0)}=0 \text { on } \gamma,
\end{array}\right.
$$


where the function $F_{N} \in L_{2}\left(\mathbb{R}_{+}^{2}\right) \cap C^{\infty}(\widetilde{B})$ has the asymptotic behaviour $F_{N}=O\left(\tau^{-2 N-3}\right)$ as $\tau \rightarrow \infty$ and is odd in $\zeta_{1}$. By Lemma 3.6 there exists a solution

$$
W_{N}^{(0)} \in H_{\mathrm{loc}}^{1}\left(\mathbb{R}_{+}^{2}\right) \cap C^{\infty}(\widetilde{B}) \cap H_{\mathrm{loc}}^{2}\left(\mathbb{R}_{+}^{2} \backslash \overline{\left(\mathcal{O}_{\varsigma,+} \cup \mathcal{O}_{\varsigma,-}\right)}\right)
$$

of the problem (326) that is odd in $\zeta_{1}$ and has the asymptotic expansion

$$
W_{N}^{(0)}=\sum_{j=0}^{N-1} \widetilde{C}_{2 j+1} \tau^{-2 j-1} \cos (2 j+1) \theta+O\left(\tau^{-2 N-1}\right) \quad \text { as } \quad \tau \rightarrow \infty .
$$

Consequently, by (325) the function $W_{N}$ is odd. We claim that the function $W_{N}$ is independent of $N$. Let $N_{1} \geq N \geq 4$ and let $W^{\left(N, N_{1}\right)}=W_{N}-W_{N_{1}}$. Then for $W^{\left(N, N_{1}\right)}$ we obtain the boundary-value problem

$$
\left\{\begin{array}{l}
\Delta_{\xi} W^{\left(N, N_{1}\right)}=0 \quad \text { in } \mathbb{R}_{+}^{2}, \\
\frac{\partial W^{\left(N, N_{1}\right)}}{\partial \zeta_{2}}=0 \quad \text { on } \Gamma, \\
W^{\left(N, N_{1}\right)}=0 \quad \text { on } \gamma,
\end{array}\right.
$$

and the function $W^{\left(N, N_{1}\right)}$ decreases as $\tau \rightarrow \infty$. Then by the uniqueness of the solution of this boundary-value problem, the function satisfies $W^{\left(N, N_{1}\right)} \equiv 0$ for $\zeta_{2} \geq 0$ (see, for example, 75) and, consequently, $W_{N}$ is independent of $N$. As $N$ is arbitrary, by (325), (327), and the theorem on the increase of smoothness, it follows that $W \in B_{\text {odd }}^{(2 k+1,2 n+1,1)}$. The lemma is proved.

The following result is proved completely analogously.

Lemma 4.9. Let $F \in B_{\text {even }}^{(2 k, 2 n, 0)}$ and let $Q$ be a function in $\bar{F}_{\text {even }}^{(0,2 n)}$ such that

$$
F-\Delta Q=O\left(\tau^{-2}\right) \quad \text { as } \quad \tau \rightarrow+\infty .
$$

Then there exists a solution $W \in B_{\mathrm{even}}^{(2 k, 2 n, 1)}$ of the boundary-value problem

$$
\left\{\begin{array}{l}
\Delta_{\zeta} W=F \quad \text { in } \mathbb{R}_{+}^{2}, \\
\frac{\partial W}{\partial \zeta_{2}}=0 \quad \text { on } \Gamma, \\
W=0 \quad \text { on } \gamma, \\
W \sim Q \quad \text { as } \tau \rightarrow+\infty .
\end{array}\right.
$$

Proof of Theorem 3.6. The proof of the theorem follows from the definition of the spaces $\mathbb{B}^{(k, n, 0)}, F^{(1, k)}$ and the statements of Lemmas 4.8 and 4.9 .

\section{ACKNOWLEDGEMENTS}

The author is grateful to R. R. Gadyl'shin for his interest in these results and for the useful advice and comments he gave.

\section{REFERENCES}

1. A. N. Krylov, Some differential equations of mathematical physics having applications in technical questions, Reports of the Nikolaev Naval Academy, no. 2 (1913), 325-348. (Russian)

2. E. Sánchez-Palencia, Perturbation of eigenvalues in thermoelasticity and vibration of systems with concentrated masses, Trends and Applications of Pure Mathematics to Mechanics, Lecture Notes in Phys., vol. 195, Springer-Verlag, Berlin, 1984, pp. 346-368. MR755735 (85m:73010)

3. O. A. Oleĭnik, Lectures on partial differential equations, Binom, Moscow, 2005. (Russian)

4. O. A. Oleurnik, On the eigenoscillations of bodies with concentrated masses, Current problems of applied mathematics and mathematical physics, Nauka, Moscow, 1988, pp. 101-128. (Russian) MR.990734 (90c:35020)

5. O. A. Oleĭnik, On spectra of some singularly perturbed operators, Uspekhi Mat. Nauk 42, no. 3 (1987), 221-222; English transl. Russian Math. Surveys, 42 (1987), 3. 
6. O. A. Oleŭnik, Homogenization problems in elasticity. Spectra of singularly perturbed operators, Nonclassical continuum mechanics (Durham, 1986), London Math. Soc. Lecture Note Ser. vol. 122, Cambridge Univ. Press, Cambridge, 1987, pp. 53-95. MR.926498 (89c:73022)

7. O. A. Oleŭnik, On frequencies of eigenoscillations of bodies with concentrated masses, Functional and numerical methods in mathematical physics, Naukova Dumka, Kiev, 1988, pp. 165-171. (Russian) MR.1038572(91c:35012)

8. Yu. D. Golovatyı̆, Spectral properties of oscillatory systems with attached masses, Kandidat Dissertation, Moskov. Univ., 1988. (Russian)

9. Yu. D. Golovatyı̆, S. A. Nazarov, O. A. Oleŭnik, and T. S. Soboleva, On eigenoscillations of a string with an attached mass, Sibirsk. Mat. Zh. 29, no. 5 (1988), 71-91; English transl. in Siberian Math. J. 29 (1989), 744-760. MR971229 (90e:34044)

10. O. A. Oleı̆nik and T. S. Soboleva, On eigenoscillations of a nonhomogeneous string with a finite number of attached masses, Uspekhi Mat. Nauk 43, no. 4 (1988), 187-188; English transl. in Russian Math. Surveys, 43, no. 4 (1988).

11. Yu. D. Golovatyı̆, On eigenoscillations and eigenfrequencies of an elastic rod with an attached mass, Uspekhi Mat. Nauk 43, no. 4 (1988), 163-192; English transl. Russian Math. Surveys, 43, no. 4.

12. Yu. D. Golovaty̌, On eigenoscillations and eigenfrequencies of a clamped plate with an attached mass, Uspekhi Mat. Nauk 43, no. 5 (1988), 185-186; English transl. in Russian Math. Surveys 43, no. 5 (1988), 227-228. MR 971476 (90b:35027)

13. S. A. Nazarov, Concentrated masses problems for a spatial elastic body, C. R. Acad. Sci. Paris Sér. I Math. 316 (1993), 627-632. MR1212218(94b:73017)

14. I. I. Argatov and S. A. Nazarov, Junction problem of shashlik (skewer) type, C. R. Acad. Sci. Paris Sér. I Math. 316 (1993), 1329-1334. MR1226125 (94e:35051)

15. Yu. D. Golovatyı̆, Spectral properties of oscillatory systems with attached masses, Trudy Moskov. Mat. Obshch. 54 (1992), 29-72; English transl. in Trans. Moscow Math. Soc. 1993 (1993), 23-59. MR.1256922 (95b:73012)

16. Yu. D. Golovatyı̆, The spectral Neumann problem for the Laplace operator with singularly perturbed density, Uspekhi Mat. Nauk 45, no. 4 (1990), 147-148; English transl. in Russian Math. Surveys 45, no. 4 (1990), 165-167. MR 1075393 (92g:35162)

17. S. A. Nazarov, On a problem of Sánchez-Palencia with Neumann boundary conditions, Izv. Vyssh. Uchebn. Zaved. Mat. 1989, no. 11 (1989), 60-66; English transl. in Soviet Math. (Iz. VUZ) 33, no. 11 (1989), 73-78. MR.1045104 (91k:35187)

18. N. U. Rakhmanov, On eigenoscillations of systems with concentrated masses, Kandidat Dissertation, Moskov. Univ., 1991. (Russian)

19. O. A. Oleĭnik, G. A. Iosif'yan, and A. S. Shamaev, Mathematical problems in the theory of strongly inhomogeneous elastic media, Moscow Univ., Moscow, 1990. English translation, Mathematical problems in elasticity and homogenization, Elsevier, Amsterdam, 1992. MR.1115306 (92i:73009)

20. Yu. D. Golovaty functions in problems on oscillations of a medium with singular perturbation of the density, Uspekhi Mat. Nauk 43, no. 5 (1988), 189-190; English transl. in Russian Math. Surveys 43, no. 5 (1988), 229-230. MR971478 (89k:35172)

21. Yu. D. Golovaty̆, S. A. Nazarov, and O. A. Oleı̆nik, Asymptotic expansions of eigenvalues and eigenfunctions of problems on oscillations of a medium with concentrated perturbations, Trudy Mat. Inst. Steklov. 192 (1990), 42-60; English transl. in Proc. Steklov Inst. Math. 1992, no. 3 (1993), 43-63. MR.1097888 (92b:35113)

22. E. Sánchez-Palencia and H. Tchatat, Vibration de systèmes élastiques avec des masses concentrées, Rend. Sem. Mat. Univ. Politec. Torino 42, no. 3 (1984), 43-63. MR834781 (87i:73039)

23. Y. D. Golovaty and A. S. Lavrenyuk, Asymptotic expansions of local eigenvibrations for plate with density perturbed in neighbourhood of one-dimensional manifold, Mat. Stud. 13 (2000), 51-62. MR.1777323(2001k:74053)

24. C. Leal and J. Sanchez-Hubert, Perturbation of the eigenvalues of a membrane with a concentrated mass, Quart. Appl. Math. 47 (1989), 93-103. MR987898(90c:73077)

25. M. Lobo and E. Pérez Asymptotic behavior of the vibrations of a body having many concentrated masses near the boundary, C. R. Acad. Sci. Paris Sér. II 314 (1992), 13-18.

26. M. Lobo and E. Pérez, On vibrations of a body with many concentrated masses near the boundary, Math. Models Methods Appl. Sci. 3 (1993), 249-273. MR1212942 (94h:73034)

27. M. Lobo and E. Pérez, Vibrations of a body with many concentrated masses near the boundary: High frequency vibrations, Spectral analysis of complex structures (Paris, 1993), Travaux en Cours vol. 49, Hermann, Paris, 1995, pp. 85-101. MR.1488737(98j:73037) 
28. M. Lobo and E. Pérez, Vibrations of a membrane with many concentrated masses near the boundary, Math. Models Methods Appl. Sci. 5 (1995), 565-585. MR1347148 (96g:73025)

29. M. Lobo and E. Pérez, High frequency vibrations in a stiff problem, Math. Models Methods Appl. Sci. 7 (1997), 291-311. MR.1440610 (98e:35053)

30. M. Lobo and E. Pérez A skin effect for systems with many concentrated masses, C. R. Acad. Sci. Paris Sér. IIb 327 (1999), 771-776.

31. D. Gómez, M. Lobo, and E. Pérez, On the eigenfunctions associated with the high frequencies in systems with a concentrated mass, J. Math. Pures Appl. (9) 78 (1999), 841-865. MR1715344 (2000h:35119)

32. M. Lobo and E. Pérez, The skin effect in vibrating systems with many concentrated masses, Math. Methods Appl. Sci. 24 (2001), 59-80. MR1809494 (2001m:35029)

33. O. A. Oleinik, J. Sanchez-Hubert, and G. A. Yosifian, On vibrations of a membrane with concentrated masses, Bull. Sci. Math. 115 (1991), 1-27. MR.1086936 (92a:73021)

34. J. Sanchez-Hubert and E. Sánchez-Palencia, Vibration and coupling of continuous systems. Asymptotic methods, Springer-Verlag, Berlin, 1989. MR996423 (91c:00018)

35. J. Sanchez-Hubert, Perturbation des valeurs propres pour des systèmes avec masse concentrée, C. R. Acad. Sci. Paris Sér. II 309 (1989), 507-510. MR.1022289 (90j:35151)

36. N. O. Babich and Yu. D. Golovatǐ̌, On the Neumann spectral problem for a singular perturbed differential operator of the fourth order, Visn. L'viv. Univ. Ser. Mekh.-Mat. 51 (1998), 118-127. (Ukrainian)

37. N. O. Babich, High-frequency asymptotics of global vibrations in a problem with a locally perturbed density, Mat. Metodi Fiz.-Mekh. Polya 42, no. 3 (1999), 36-44. (Ukrainian) MR1977858 (2003m:74095)

38. Yu. D. Golovatiur and A. Golovach, On the asymptotics of global eigenoscillations of a strongly nonhomogeneous string, Visn. L'viv Univ. Ser. Mekh.-Mat. 48 (1997), 88-99. (Ukrainian)

39. Yu. D. Golovatiı and V. M. Flyud, On interaction of local and global oscillations of a strongly nonhomogeneous string, Proc. Int. Sci. Conf. "Current problems of mathematics", Chernavtsi-Kiev, 1998, part 1, pp. 138-141. (Ukrainian)

40. G. Grabchak, The spectral Neumann problem for a system of equations in the linear theory of elasticity with a singular density distribution, Visn. L'viv Univ. Ser. Mekh.-Mat. 45 (1996), 124140. (Ukrainian)

41. Yu. D. Golovatyj, On WKB-approximation of high frequency vibrations of a singular perturbed string, Proc. Int. Conf. "Nonlinear partial differential equations", Kiev, 1997, p. 62.

42. T. A. Mel'nyk, Vibrations of a thick periodic junction with concentrated masses, Math. Models Methods Appl. Sci. 11 (2001), 1001-1027. MR1850560 (2002f:35024)

43. T. A. Mel'nyk, Vibrations and pseudovibrations of thick periodic junctions with concentrated masses, Dopov. Nats. Akad. Nauk Ukr. Mat. Prirodozn. Tekh. Nauki 2001, no. 9 (2001), 47-53. MR1886107 (2002i:35021)

44. E. I. Doronina and G. A. Chechkin, On eigenoscillations of a body with many concentrated masses located nonperiodically along the boundary, Trudy Mat. Inst. Steklova 236 (2002), 158-166; English transl. in Proc. Steklov Inst. Math. 2002, no. 1 (2002), 148-156 MR.1931016 (2003d:74034)

45. V Rybalko, Vibrations of elastic systems with a large number of tiny heavy inclusions, Asymptot. Anal. 32 (2002), 27-62. MR.1943039 (2004a:74029)

46. G. A. Chechkin, E. Pérez and E. I. Yablokova, Non-periodic boundary homogenization and "light" concentrated masses, Indiana Univ. Math. J. 54 (2005), 321-348. MR2136812 (2006a:35013)

47. G. A. Chechkin, On the estimation of solutions of boundary value problems in domains with concentrated masses periodically distributed along the boundary. The case of "light" masses, Mat. Zametki 76 (2004), 928-944; English transl. in Math. Notes 76 (2004), 865-879. MR2127504 (2005m:35015)

48. G. A. Chechkin, On oscillations of a body with concentrated masses situated on the boundary, Uspekhi Mat. Nauk 50, no. 4 (1995), 105-106; English transl. in Russian Math. Surveys 50 (1995), 763-764.

49. G. A. Chechkin, On the vibration of a partially fastened membrane with many 'light' concentrated masses on the boundary, C. R. Mécanique 332 (2004), 949-954.

50. G. A. Chechkin, The splitting of a multiple eigenvalue in the problem of concentrated masses, Uspekhi Mat. Nauk 59, no. 4 (2004), 205-206; English transl. in Russian Math. Surveys 59, no. 4 (2004), 790-791. MR2106656

51. G. A. Chechkin, Asymptotic expansions of the eigenvalues and eigenfunctions of an elliptic operator in a domain with many "light" concentrated masses near the boundary. The two-dimensional case, Izv. Ross. Akad. Nauk Ser. Mat. 69, no. 4 (2005), 161-204; English transl. in Izv. Math. 69, no. 4 (2005), 805-846. MR2170707(2006h:35006) 
52. G. A. Chechkin, Asymptotic expansions of eigenelements of the Laplace operator in a domain with many "light" concentrated masses closely located on the boundary. Multi-dimensional case, Probl. Mat. Analiz. 30 (2005), 87-119; English transl. in J. Math. Sci. (N. Y.) 128 (2005), 3263-3305. MR2171602 (2006m:35056)

53. G. A. Chechkin, Boundary homogenization in domains with singular density, Differentsial'nye Uravneniya 39 (2003), 855; English transl. in Differential Equations, 39(2003), 904.

54. G. A. Chechkin, Homogenization of solutions to problems for the Laplace operator in unbounded domains with many concentrated masses on the boundary, Problems in mathematical analysis, 33 (2006), 103-111; English transl. in Math. Sci. (N. Y.) 139 (2006), 6351-6362. MR2278909 $(2007 \mathrm{k}: 35019)$

55. A. M. Il'in, Matching of asymptotic expansions of solutions of boundary value problems, Nauka, Moscow, 1989; English transl., Transl. Math. Monographs vol. 102, Amer. Math. Soc., Providence, RI, 1992. MR:1007834 (90i:35062)

56. R. R. Gadyl'shin, Asymptotics of the eigenvalues of a boundary value problem with rapidly oscillating boundary conditions, Differentsial'nye Uravneniya 35 (1999), 540-551; English transl. in Differential Equations 35 (1999), 540-551. MR1719780 (2000j:35212)

57. G. A. Chechkin, Averaging of boundary value problems with singular perturbation of the boundary conditions, Mat. Sb. 184, no. 6 (1993), 99-150; English transl. in Russian Acad. Sci. Sb. Math. 79 (1994), 191-222. MR1234592(94j:35014)

58. D. I. Borisov, On a boundary value problem in a cylinder with a partial change of type of boundary conditions, Mat. Sb. 193, no. 7 (2002), 37-68; English transl. in Sb. Math. 193 (2002), 977-1008. MR 1936849 (2003i:35211)

59. S. L. Sobolev, Some applications of functional analysis in mathematical physics, Nauka, Moscow, 1988; English transl., Transl. Math. Monographs vol. 90, Amer. Math. Soc., Providence, RI, 1991. MR.986735 (90m:46059)

60. S. L. Sobolev, Selected problems in the theory of function spaces and generalized functions, Nauka, Moscow, 1989. (Russian) MR993984 (90m:46060)

61. O. A. Ladyzhenskaya, The boundary value problems of mathematical physics, Nauka, Moscow, 1973; English transl., Applied Mathematical Sciences vol. 49, Springer-Verlag, New York, 1985. MR.0599579 (58:29032)

62. V. P. Mikhaǔlov, Partial differential equations, Nauka, Moscow, 1983; English transl., Mir, Moscow, 1978.

63. R. R. Gadyl'shin, Asymptotics of the eigenvalue of a singularly perturbed selfadjoint elliptic problem with a small parameter in the boundary conditions, Differentsial'nye Uravneniya 22 (1986), 640-652; English transl. in Differ. Equations 22 (1986), 474-483. MR843222(87m:35167)

64. R. R. Gadyl'shin, Splitting of a multiple eigenvalue of the Dirichlet problem for the Laplace operator under singular perturbation of the boundary condition, Mat. Zametki 52, no. 4 (1992), 42-55; English transl. in Math. Notes 52 (1993), 1020-1029. MR.1203951 (94c:35132)

65. D. I. Borisov, On a model boundary value problem for Laplacian with frequently alternating type of boundary condition, Asymptot. Anal. 35 (2003), 1-26. MR2004716 (2005g:35009)

66. M. Yu. Planida, On the convergence of solutions of singularly perturbed boundary value problems for the Laplacian, Mat. Zametki 71 (2002), 867-877; English transl. in Math. Notes 71 (2002), 794-803. MR 1933107 (2003g:35006)

67. M. Yu. Planida, Asymptotics of eigenvalues for a cylinder that is heat-insulated on a narrow strip, Zh. Vychisl. Mat. Mat. Fiz. 43 (2003), 422-432; English transl. in Comput. Math. Math. Phys. 43 (2003), 403-413. MR1994401(2004e:35171)

68. A. M. Il'in, A boundary value problem for an elliptic equation of second order in a domain with a narrow slit. I. The two-dimensional case, Mat. Sb. 99 (1976), 514-537; English transl. in Math. USSR-Sb. 28 (1976), 459-480. MR0407439 (53:11214)

69. A. M. Il'in, A boundary value problem for an elliptic equation of second order in a domain with a narrow slit. II. Domain with a small cavity, Mat. Sb. 103 (1977), 265-284; English transl. in Math. USSR-Sb. 32 (1977), 227-244. MR0442460 (56:842)

70. A. M. Il'in, Study of the asymptotic behavior of the solution of an elliptic boundary value problem in a domain with a small hole, Trudy Sem. Petrovsk. 6 (1981), 57-82; English transl. in J. Soviet Math. 33 (1986), 994-1014. MR630701 (83e:35045)

71. R. R. Gadyl'shin, Asymptotics of the minimum eigenvalue for a circle with fast oscillating boundary conditions, C. R. Acad. Sci. Paris Sér. I Math. 323 (1996), 319-323. MR1404781 (97f:35158)

72. R. R. Gadyl'shin, A boundary value problem for the Laplacian with rapidly oscillating boundary conditions, Dokl. Akad. Nauk 362 (1998), 456-459; English transl. in Dokl. Math. 58 (1998), 293296. MR:1708976 (2000g:35034) 
73. R. R. Gadyl'shin, The splitting of a multiple eigenvalue in a boundary value problem for a membrane clamped to a small section of the boundary, Sibirsk. Mat. Zh. 34, no. 3 (1993), 43-61; English transl. in Siberian Math. J. 34 (1993), 433-450. MR1241167 (94k:35219)

74. S. A. Nazarov and B. A. Plamenevskiǔ, Elliptic problems in domains with piecewise smooth boundaries, Nauka, Moscow, 1991; English transl., De Gruyter Expositions in Mathematics vol. 13, de Gruyter, Berlin, 1994. MR1283387 (95h:35001)

75. M. A. Lavrent'ev and B. V. Shabat, Methods in the theory of functions of a complex variable, Nauka, Moscow, 1987. (Russian) MR1087298 (91k:30003)

Moscow State University, Moscow, Russia

E-mail address: chechkin@mech.math.msu.su 